LBL-33932

$\mathrm{UC}-408$

\title{
Spectroscopic Studies of Synechococcus sp PCC 7002 Phycobilisome Core Mutants
}

Yvonne Marie Gindt

(Ph. D. Thesis)

\author{
Department of Chemistry \\ University of California \\ and \\ Structural Biology Division \\ Lawrence Berkeley Laboratory \\ University of California \\ Berkeley, CA 94720
}

April 1993

This work was supported by the Director, Office of Energy Research, Office of Basic Energy Sciences, of the U.S. Department of Energy under Contract No. DE-AC03-76SF00098 


\author{
Abstract \\ Spectroscopic Studies of Synechococcus sp PCC 7002 \\ Phycobilisome Core Mutants \\ b y \\ Yvonne Marie Gindt \\ Doctor of Philosophy in Chemistry \\ University of California at Berkeley \\ Professor Ken Sauer, Chair
}

The role of the $\mathrm{L}_{\mathrm{cm}}, \beta^{18}$, and $\alpha^{\mathrm{AP}-\mathrm{B}}$ chromoproteins in the phycobilisome core is investigated in this dissertation. Genetically engineered strains of Synechococcus sp PCC 7002 missing different polypeptides and/or chromophores were obtained for this purpose. Intact cells, isolated PBS, and subcore preparations for each mutant were studied to determine the effect of that mutation on energy transfer within the PBS core and from the PBS to the reaction centers. Three mutants lacked the $\beta^{18}$ and/or $\alpha^{\text {AP-B }}$ polypeptides, while the $\mathrm{L}_{\mathrm{cm}}$ chromophore was altered in the other two mutants. A lower energy absorbing chromophore, $A_{\max }=695 \mathrm{~nm}$, was substituted for the $\mathrm{L}_{\mathrm{cm}}$ chromophore.

The deletion of the $\beta^{18}$ and $\alpha^{A P-B}$ subunits had no discernible effect on energy transfer from the PBS to PSII within the cells. In the cells and the isolated PBS, the altered $\mathrm{L}_{\mathrm{cm}}$ chromophore acts to both quench the PBS complex and to redirect the energy which would be transferred to PSII.

In the PBS and subcore preparations, the deletion of the $\alpha^{\mathrm{AP}-\mathrm{B}}$ subunit did not alter energy transfer within the core. The deletion 
of the $\beta^{18}$ subunit from the PBS caused a small decrease in the excited state lifetimes of the final emitters indicating more disorder within the core.

The $\mathrm{L}_{\mathrm{cm}}$ chromophore was found to absorb at $670 \mathrm{~nm}$ and to emit at $683 \mathrm{~nm}$ within the intact PBS. The $\beta^{18}$ chromophore emits at $679 \mathrm{~nm}$ while the $\alpha$ AP-B chromophore emits at $682 \mathrm{~nm}$. A strong interaction exists between the $L_{c m}$ chromophore and the $\beta^{18}$ subunit. Upon deletion of the $\beta^{18}$ subunit from the PBS core, the $L_{\mathrm{cm}}$ chromophore emits at a higher energy. The $\beta^{18}$ subunit could act to stabilize the $\mathrm{L}_{\mathrm{cm}}$ chromophore-binding pocket, or exciton coupling could be occurring between the two chromophores.

The role of the $\alpha$ AP-B chromophore is still unclear at this time. The $\alpha$ AP-B chromophore does contribute to the RT emission of the isolated PBS, but it transfers energy to the $L_{c m}$ at $77 \mathrm{~K}$. From this result, one can conclude that the $\alpha^{\mathrm{AP}-\mathrm{B}}$ subunit is adjacent to the trimer containing the $\mathrm{L}_{\mathrm{cm}}$ polypeptide. 
Table of Contents

Table of Contents

iii

Abbreviations

Acknowledgments

$\mathrm{v}$

vi

\section{Chapter 1 Introduction}

A. Introduction to the phycobilisome 2

B. Rod Composition 4

C. Core Substructure 9

D. Arrangement of Trimers in the Core 11

E. The $\beta^{18}$ Subunit 17

F. The $\alpha$ AP-B Subunit 18

G The $\mathrm{L}_{\mathrm{cm}}$ Polypeptide 19

H. The Project 23

I. References for Chapter 1

Chapter 2 Materials and Methods

A. The Cultures 29

B. Preparation of Intact Phycobilisomes 29

C. Preparation of the $18 \mathrm{~S}$ Core Particle 33

D. HPLC of 18 S Preparations 37

E SDS-PAGE $\quad 39$

F. Spectroscopy Sample Preparation 45

G Steady State Spectroscopy 47

I. Time-Resolved Fluore:cence Spectroscopy 48

J. Data Analysis of Time-Resolved Fluorescence Decays 51

K References for Chapter 2

Chapter 3 Discussion of the Whole-Cells Results

A. Absorption Spectroscopy 59

B. Steady State Emission 65 
C. Time-Resolved Fluorescence 71

D. Conclusions $\quad 82$

E References for Chapter $3 \quad 86$

Chapter 4 Discussion of PBS Results
A. Absorption Spectroscopy
88
B. Steady State Fluorescence
C. Low Temperature Emission 101
D. Time-Resolved Fluorescence 105
E Conclusions 116
F. References for Chapter 4 122

Chapter 5 Discussion of 18 S Preparation Results
A. Absorption Spectroscopy
125
B. Room Temperature Emission
C. Low Temperature Emission
138
D. Time-Resolved Fluorescence
142
E. Discussion
151
F. Conclusions
158
G. References for Chapter 5

Chapter 6 Conclusions and Future Work
A. The $\alpha$ AP-B Subunit
163
B. The $\beta^{18}$ Subunit
165
C. The $\mathrm{L}_{\mathrm{cm}}$ Chromophore
166
D. Energy Transfer within the Core
168
E Future Directions
168
F. References for Chapter 6
172 


\section{Abbreviations}

AP

apcD

apcDF

apcDE/C186S

apcE/C186S

chl $a$

DAS

DCM

HITC

PAGE

PC

PE

PMSF

PSI

PSII

SDS allophycocyanin

Synechococcus sp PCC 7002 mutant lacking the $\alpha$ AP-B subunit

Synechococcus sp PCC 7002 mutant lacking the $\alpha^{A P-B}$ and $\beta^{18}$ subunits

Synechococcus sp PCC 7002 mutant lacking the $\alpha$ AP-B subunit along with the substitution of serine for cysteine at position 186 on the $\mathrm{L}_{\mathrm{cm}}$ polypeptide

Synechococcus sp PCC 7002 mutant containing the substitution of serine for cysteine at position 186 on the $\mathrm{L}_{\mathrm{cm}}$ polypeptide

chlorophyll $a$

decay-associated spectra

4-dicyanomethylene-2-methylene-6-( $p$ dimethylamino styrene)-4H-pyrane

$1,1^{\prime}, 3^{\prime}, 3^{\prime}, 3^{\prime}$-hexamethylindotricarbocyanine polyacrylamide gel electrophoresis phycocyanin phycoerythrin phenylmethylsulfonyl fluoride photosystem I photosystem II sodium dodecyl sulfate 


\section{Acknowledgments}

Most of all, I would like to thank Ken Sauer for inviting me into his group and for providing me with good advice, cool instruments, and money. I have never regretted being part of the Sauer group. I would also like to thank all the other people in the Sauer/Klein group for providing me with technical advice, practice orals and GRCs, and friendship. I especially would like to acknowledge Mary Talbot and Martin Debreczeny for all the help they have given me throughout these five years. Ishita Mukerji deserves a special mention for her patience in teaching single photon timing spectroscopy.

I also want to thank all the Calvin Lab building staff for keeping - my research going including Gary Smith and Vangie Peterson. I really value their commitment to Calvin Lab.

I want to acknowledge the support of Don Bryant and Jianhui Zhou. This project would not have been possible without their efforts. I would like to thank Crystal and Craig over in Stanley Hall for helping me with biochemistry and HPLC. They proved to be very knowledgeable and helpful. I would like to acknowledge Alex Glazer for providing advice and access to his laboratory.

I want to thank all my friends inside and outside the lab for supporting my efforts with a special mention of my roomie and running partner and soccer teammate-Robin. I would like to thank my running and soccer buddies--Kerstin, Brenda, Nancy, Tina, Andy, Ben. I would like to acknowledge past Bruisers--Sandra, 
Margo, Judy, Corinna, and all the present Bruisers, especially Jim. I will really miss all of you.

I want to thank my mom, sisters, and brothers for supporting my efforts. Mom, this Ph.D. is for you (even if you don't understand my research).

Last, but not least, I would like to thank the Department of Energy for making all this possible. Funding for this work was provided by the Director, Office of Energy Research, Office of Basic Energy Sciences, Energy Biosciences Division, of the US Department of Energy under Contract No. DE-AC03-76SF00098. 


\section{Chapter 1. Introduction to the Phycobilisome}

"...the sun is not only the author of visibility in all visible things, but of generation and nourishment and growth..."

Plato, The Republic

Humankind has always had a deep fascination and respect for the process of photosynthesis, and during the last century we are finally solving the mystery of what drives all living things on earth. Many of the processes involved in photosynthesis are starting to be understood in great detail. For example, in the last 10 years crystals of the purple bacterial reaction center and Photosystem I have been grown and structures determined (Deisenhofer, Epp et al., 1984; Witt, Krauss et al., 1992). With the crystal structures known, the research on the reaction centers has accelerated to an overwhelming rate.

As reaction centers become better understood we need to study other aspects of photosynthesis so that in the future we may be able to develop efficient artificial photosynthesis. One major problem that needs to be solved for efficient artificial photosynthesis is how to collect photons and transfer their energy with almost $100 \%$ efficiency to the reaction centers. Plants, algae, and photosynthetic bacteria have solved this problem using a variety of methods, none of which is fully understood at this time (Glazer, 1983). 
I would like to address the questions of how light energy is able to reach the reaction centers from outlying light-harvesting antennas with greater than $90 \%$ efficiency, and the importance of the chromophore placement in the process of the energy transfer. The light harvesting antenna under scrutiny is that found in cyanobacteria and red algae: the phycobilisome.

First, I will discuss the function and the structure of the phycobilisome as it is found in the cyanobacterium, Synechococcus sp PCC 7002 (also known as Agmenellum quadruplicatum), and I will describe the mutants under study. I will then follow with a methods and materials chapter, three chapters of results, and finally, a discussion of my results and future experiments.

\section{Introduction to the Phycobilisome}

The phycobilisome (PBS) is a light-harvesting antenna complex found in cyanobacteria and red algae (Bryant, 1991; Glazer, 1989; Glazer, 1985; Glazer, 1984; Gantt, 1981; Gantt, 1980). Calculations indicate that it transfers light energy with greater than $95 \%$ efficiency to reaction centers where the actual charge separation of photosynthesis takes place (Porter, Tredwell et al., 1978). The cyanobacterium gains a tremendous competitive advantage having this antenna because PBS absorb light in the part of the visible spectrum where chlorophyll $a$ (chl $a$ ) and carotenoid absorption is at a minimum. Chl $a$ absorbs photons below $450 \mathrm{~nm}$ and above $650 \mathrm{~nm}$, while carotenoids usually absorb from 400 to $500 \mathrm{~nm}$. Thus the phycobilisome antenna is able to collect photon energy 
from 500 to $650 \mathrm{~nm}$ that would normally escape the reaction centers.

The PBS appears to serve other functions also: for example, it can be a source of nitrogen and carbon when the cells are starving (Bryant, 1991; Stevens, Balkwill et al., 1981). I will focus solely on the light harvesting aspects of the PBS, but it is important to be aware of the nutritional role of the PBS in the cyanobacterium.

The PBS transfers most of the energy it collects to Photosystem II (Kura-Hotta, Satoh et al., 1986; Manodori and Melis, 1985; Clement-Metral and Gantt, 1983; Katoh and Gantt, 1979). Recently, independent experiments carried out by Mullineaux and Bryant indicate that energy is transferred to PSI from PBS depending upon the state (State I/State II) of the photosystems (Mullineaux, 1992; Bryant, personal communication). There appears to be a mechanism for energy transfer to PSI; when PSII is getting too many photons some of the energy is 'spilled over' to PSI with some indications of the PSII reaction center becoming uncoupled from the PBS at that point (Mullineaux and Holzwarth, 1990). More work needs to be done to elucidate the actual mechanism, but it seems reasonable that such a mechanism could occur based on the LHCII precedent, although it seems unlikely that the actual PBS antenna would move (Bruce, Brimble et al., 1989).

PBS are found in regular arrays on the stromal or cytoplasmic surface of the thylakoid membrane as shown by electron micrographs (Gantt, 1980). In cyanobacteria, the thylakoid membrane does not contain any grana or stacked regions as seen in higher plants, and the PBS are able to fill the entire membrane surface to a 
density of approximately 450 per $\mu \mathrm{m}^{2}$ (Dilworth and Gantt, 1981). PBS can be removed from the photosynthetic membrane by rupturing the cell walls and incubating the cell fragments with detergent (Yamanaka, Glazer et al., 1978). The intact, water-soluble PBS can then be isolated from chl $a$ and other chromoproteins using a sucrose density gradient.

The structure of the PBS is probably the best known of any light harvesting antenna. It is composed of two macrostructures, (rods and core) as shown in Figure 1-1, based upon electron micrographs of PBS (Glazer, Williams et al., 1979; Yamanaka, Glazer et al., 1978).

The rods are approximately $11.5 \mathrm{~nm}$ in diameter and of different lengths, while the core is composed of cylinders approximately $12 \mathrm{~nm}$ long and $11 \mathrm{~nm}$ in diameter as shown by the electron micrographs. The number of cylinders contained within the core differs with species from two to four. Synechococcus sp 7002 has a three cylinder core, the most common PBS type.

\section{Rod Composition}

The rods are composed of aggregates of chromoprotein, called phycobiliprotein, held together with colorless linker polypeptides (Tandeau de Marsac and Cohen-Bazire, 1977). For Synechococcus sp 7002 , the only chromoprotein found in tine rods is phycocyanin with an absorption maximum ( $\lambda_{\text {abss }}$ ) at approximately $620 \mathrm{~nm}$ and a fluorescence maximum $\left(\lambda_{F}\right)$ around $650 \mathrm{~nm}$. Other chromoproteins found in rods of PBS from other species include phycoerythyrin 


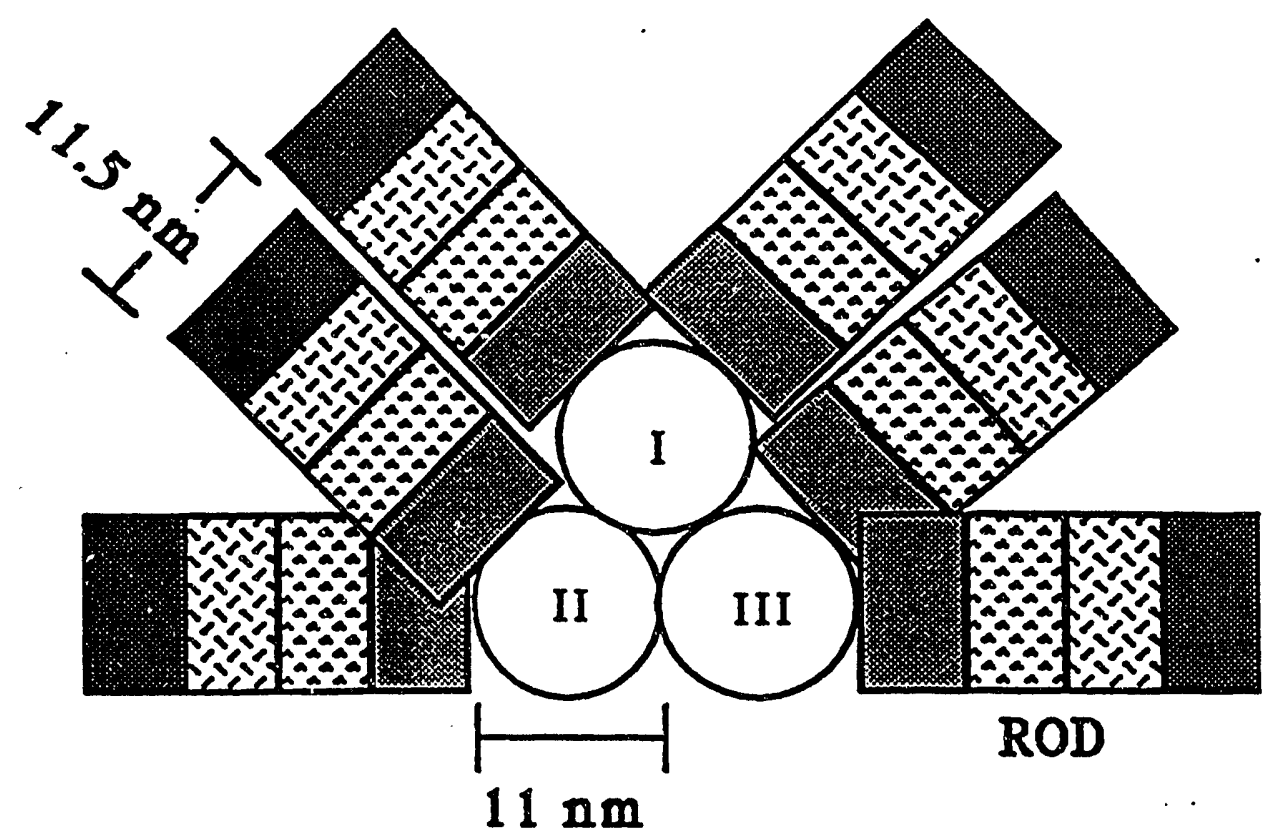

CORB

Figure 1-1. Schematic of a Synechococcus sp PCC 7002 phy cobilis o me

The PBS is composed of two substructures, rods and core. Rods are made up of hexamers of PC. The different shading in the rods correspond to different linker polypeptides present with the PC hexamer. The rods are $11.5 \mathrm{~nm}$ in diameter. The core is made up of three cylinders with each cylinder containing four trimers of AP. The core cylinders are $12 \mathrm{~nm}$ long and $11 \mathrm{~nm}$ in diameter. The schematic is based upon electron micrographs of PBS (Glazer, Williams et al., 1979, Yamanaka, Glazer et al., 1978). See Figure 1-3 for more information on the composition of both the rods and the core. 
$\left(\lambda_{\mathrm{abs}}=500\right.$ to $\left.565 \mathrm{~nm}\right)$ and phycoerythrocyanin $\left(\lambda_{\mathrm{abs}}=568\right.$ to 600 $\mathrm{nm})$.

The phycobiliproteins found in the rods are arranged in hexamers, with the placement and spectral properties of each hexamer strongly influenced by the linker polypeptide associated with it (Yu and Glazer, 1982; Lundell, Williams et al., 1981; de Lorimier, Guglielmi et al., 1990). The colorless linker polypeptides appear to finetune the spectral properties of the chromophores such that the PC hexamers on the rod periphery are bluer-absorbing than the PC hexamers in the middle of the rods which are bluerabsorbing than the PC hexamers next to the cores (Glauser, Sidler et al., 1993). Using the small differences in the absorption spectra of the various components in the rods, the PBS is optimized for unidirectional energy transfer, with photon energy being transferred from the higher energy pigments to the lower energy pigments and finally, to the reaction centers.

The crystal structure for PC hexamers from Synechococcus 7002 has been determined to $3 \AA$ resolution (Schirmer, Bode et al., 1987) showing that the hexamers are made up of two trimers added faceto-face, with each trimer made up of three heterodimers, also called monomers. Each heterodimer of PC is composed of an $\alpha$ and a $\beta$ subunit, with the $\alpha$ subunit carrying one chromophore and the $\beta$ subunit two chromophores. Free phycobiliproteins in solution commonly form aggregates with two to six heterodimers, depending upon the species of origin. 
The structure of the chromophore found in PC, a tetrapyrrole called phycocyanobilin, is shown in Figure 1-2. The chromophore is held in similar geometries in all of the binding pockets of the PC subunits with the exception of the chromophore held at position 155 on the $\beta$ subunit. Ring $D$ of the chromophore on the $\beta$ subunit is twisted compared to the other two chromophores (Schirmer, Bode et al., 1987), and the propionic sidechains show large variations between the chromophores. The crystal structure of PC hexamers shows that the tetrapyrrole is held in an extended conformation with ring A covalently bound via a thioether linkage to a cysteine of the protein backbone and the pyrrole nitrogens of rings $B$ and $C$ hydrogen bonded to an aspartate carboxyl. The chromophore displays different spectral properties depending upon the conformation it assumes. For example, without the protein scaffolding to hold the chromophore in a linear configuration, the chromophore apparently assumes a helical, lockwasher conformation (Braslavsky, Holzwarth et al., 1983); in this conformation the visible absorption and the fluorescence yield of the chromophore is greatly reduced. (Scheer, 1986). Braslavsky, Holzwarth, and Schaffner were able to show that biliverdin dimethyl esters (molecules similar to the free chromophore) free in solution could be deactivated raditionlessly by ultrafast intramolecular proton transfer between the nitrogens on the $B$ and $C$ rings in addition to $\mathrm{C}-\mathrm{C}$ rotational modes. They also found that the biliverdin dimethyl ester fluorescence decay measured using single-photon-counting could be fit to four decay components with the majority of the yield coming from a 20 ps decay component. 

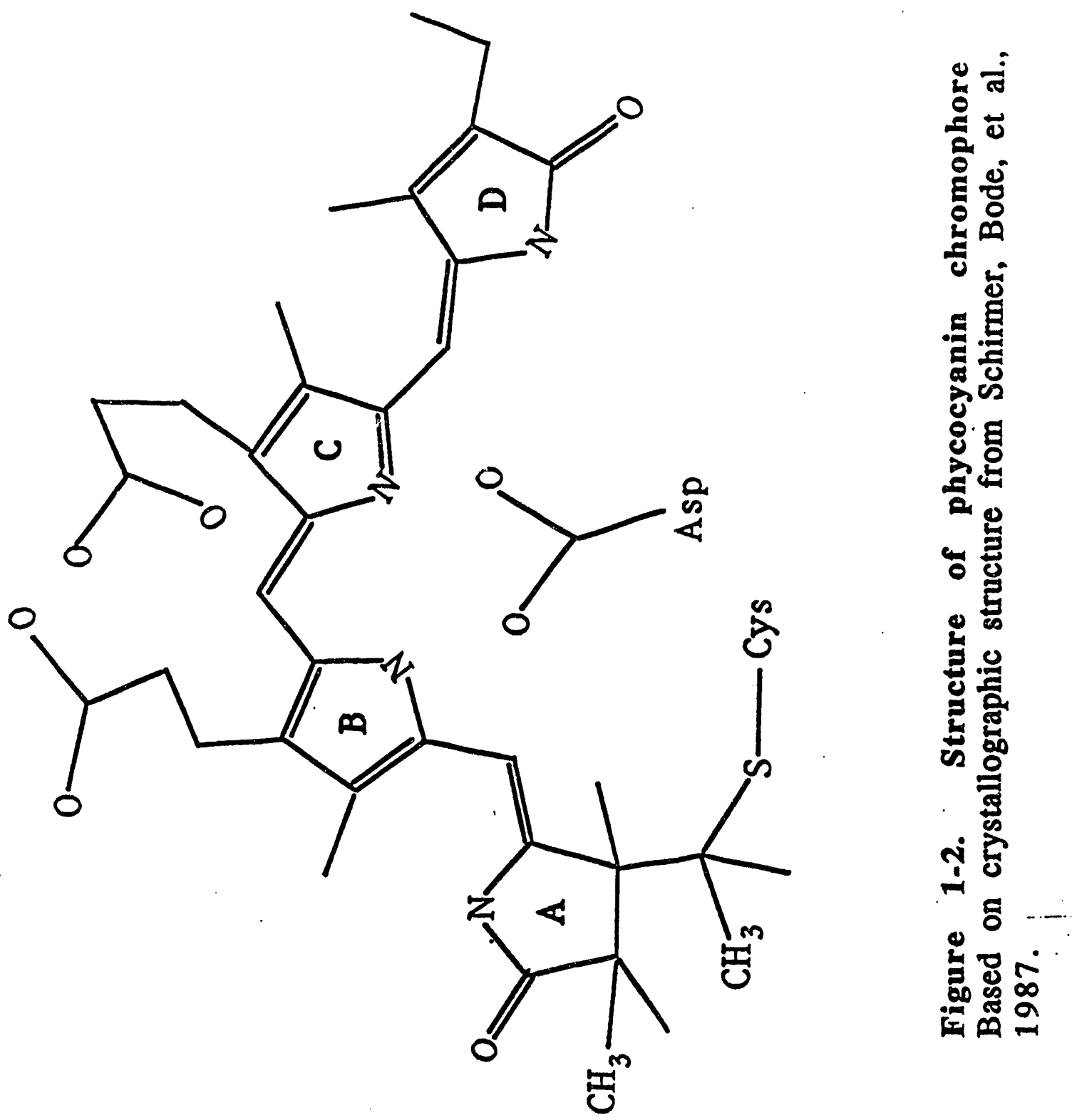
The chromophore in the denatured protein is acts in a similar manner (Scheer, 1986). This is well illustrated with the denaturation of any of the phycobiliproteins with urea at low $\mathrm{pH}$; the chromoprotein becomes very dull in color with the amount of emission significandly decreasing (Guard-Friar and MacColl, 1984). The decrease in emission is due to increased competition from radiationless deactivation as discussed above, and a concomitant decrease in lifetime would also be expected.

\section{Core Substructure}

As with the rod substructure, a hierarchy of structure exists within the cylinders of the core. Each cylinder is composed of four trimers of chromoprotein with each trimer containing three heterodimers, and each heterodimer is composed of an $\alpha$ and a $\beta$ subunit. Colorless linker polypeptides once again are involved in the ordering of the substructure as illustrated in the model shown in Figure 1-3. The small linker found in the core has also been shown to influence the spectral properties of the chromoproteins (Füglistaller, Mimuro et al., 1987).

The trimers of chromoproteins found in the core are mostly allophycocyanin (AP) with some unusual phycobiliproteins in lesser quantities. $\mathrm{AP}$ is composed of an $\alpha \beta$ heterodimer with each subunit containing only one chromophore. As with PC, the only chromophore found in the core is that shown in Figure 1-3; the protein environment apparently has a great influence on the spectral properties of the chromophore since AP trimers absorb maximally at $650 \mathrm{~nm}$ and fluoresce at $660 \mathrm{~nm}$, significantly red-shifted from 


\section{Composition of Rods}

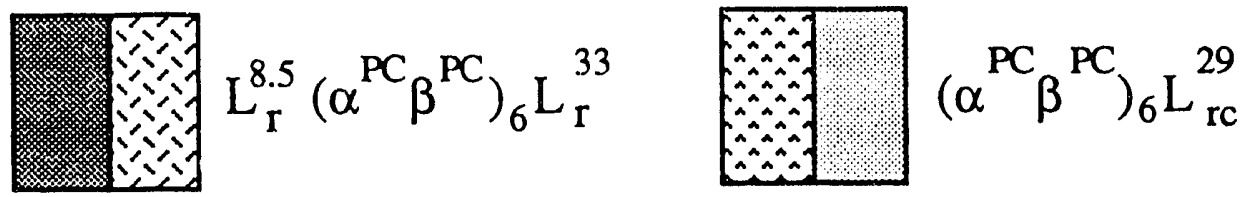

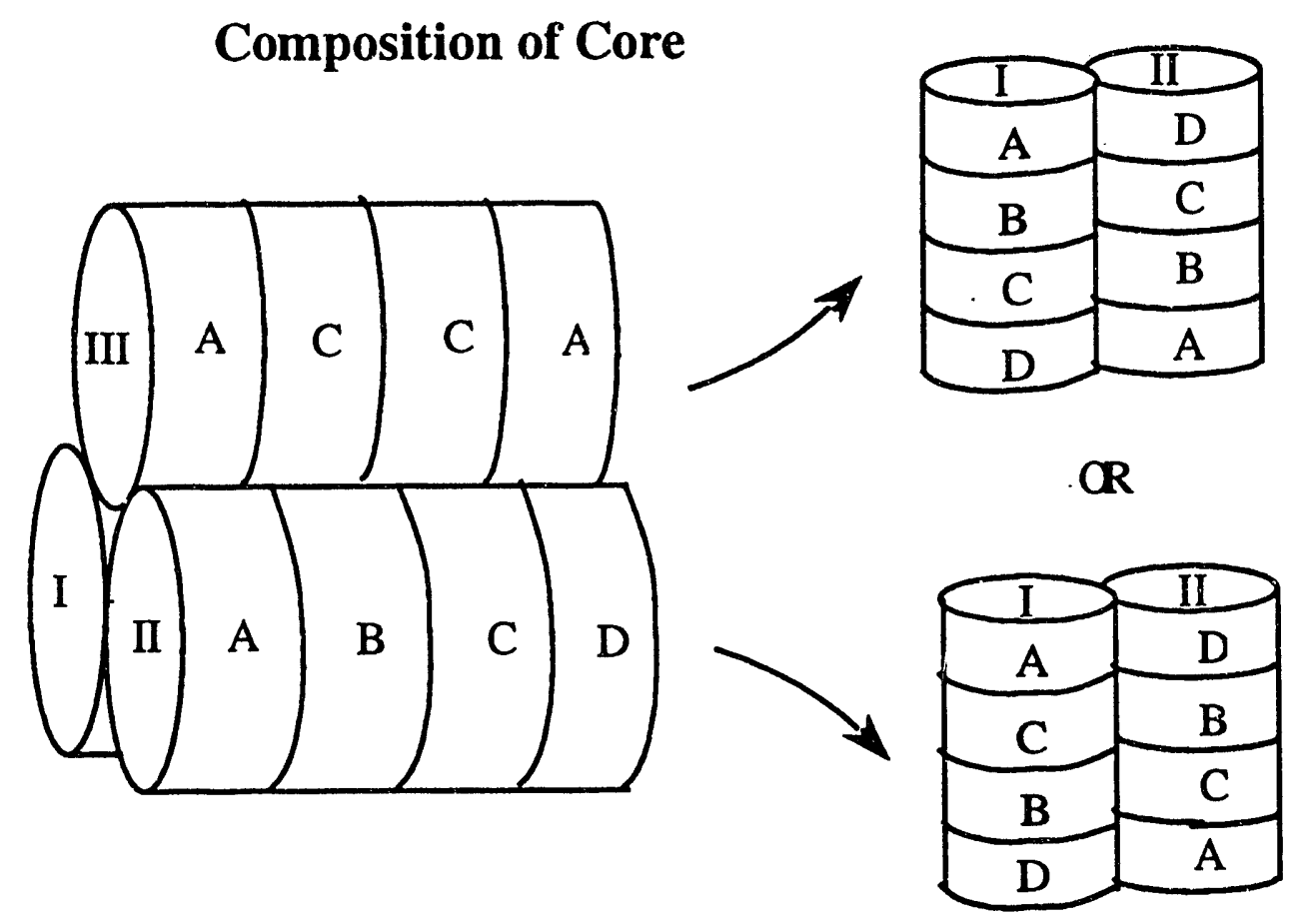
$\mathrm{A}\left(\alpha^{\mathrm{AP}} \beta \mathrm{AP}_{3}\right)_{c} \mathrm{~L}^{8}$
B $\left(\alpha^{\mathrm{AP}} \beta^{\mathrm{AP}}\right)_{2} \beta^{18} \mathrm{~L}_{\mathrm{cm}}$
$\mathrm{C}(\alpha \mathrm{AP} \beta \mathrm{AP})_{3}$
D $\left(\alpha^{\mathrm{AP}} \beta{ }^{\mathrm{AP}}\right)_{2}\left(\alpha^{\mathrm{AP}-\mathrm{B}} \beta \mathrm{AP}\right) \mathrm{L}_{c}^{8}$

Figure 1-3. Composition of phycobilisome

The molecular weight of the protein is given as a superscript with the location of the linker polypeptide given as a subscript. r: rod, rc: rod-core, c: core, cm: core-membrane, PC: phycocyanin, AP: allophycocyanin, L: linker polypeptide. The core cylinders next to the membrane are labeled I and II while the apical cylinder is labeled III. The model is from Bryant, 1988. 
that of PC hexamers $\left(\lambda_{\mathrm{abs}}=620, \lambda_{\mathrm{F}}=650 \mathrm{~nm}\right)$. At this point, one can begin to see how the PBS has been optimized for efficient energy transfer; the absorption maxima of the AP trimers overlap the fluorescence maxima of the PC hexamers found in the rods. By making small changes in the absorption spectra of the phycobiliproteins found in the antenna, the cyanobacterium ensures that the energy gets funnelled to its reaction centers quickly with little leakage.

The crystal structure of AP has yet to be determined, but due to significant amounts of sequence homology between $\mathrm{PC}$ and $\mathrm{AP}$, it is expected to be similar to that of PC.

\section{Arrangement of Trimers in the Core}

As noted before, the core is not composed solely of AP trimers; it also contains some trimers with unusual phycobiliproteins similar in amino acid composition to AP subunits but with altered spectral properties. The unusual chromoproteins including $\alpha A P-B, L_{c m}$, and possibly the $\beta^{18}$ subunit are thought to mediate energy transfer between the PBS and chl $a$ of reaction centers. I will refer to the final energy acceptors in the PBS antenna as terminal emitters. A schematic of the PBS core is given in Figure 1-3, but the arrangement of the trimers relative to each other is still under discussion. To facilitate the discussion of the core organization, I will refer to the large, chromophore-containing protein found in the core as $L_{c m}$ and the $\beta^{A P}$-like subunit found with it as the $\beta^{18}$ 
subunit. Of course, the molecular weight of these polypeptides differs somewhat among species.

Extensive work was carried out in A. Glazer's laboratory at the University of California to determine the content and arrangement of core polypeptides using PBS from Synechococcus 6301 AN112, a mutant cyanobacterium containing a stripped-down version of PBS with cores consisting of only two cylinders and rods of only one PC hexamer in length (Lundell, Williams et al., 1981; Lundell, Yamanaka et al., 1981; Yamanaka, Lundell et al., 1982 ; Lundell and Glazer, 1983a; Lundell and Glazer, 1983b; Lundell and Glazer, 1983c). In this very complete study, they dissociated the core cylinders into individual trimers and then separated the trimers using column chromatography and ultracentrifugation. They then carried out SDS-PAGE and isoelectric focusing to identify and quantify the proteins present in the trimers.

In a similar study, using a three cylinder core PBS from Synechocystis 6701, Glazer's laboratory was able to show that most of the work on the two cylinder core PBS from Synechococcus 6301 seems to correspond well to three cylinder core species of PBS (Gingrich, Lundell et al.,1983). The third (apical) cylinder appears to be composed of two $\left(\alpha \mathrm{AP}_{\beta} \mathrm{AP}\right)_{3}$ trimers and two $\left(\alpha \mathrm{AP}_{\beta} \mathrm{AP}_{3}\right)_{3} \mathrm{~L}^{10}$ trimers. Table 1-1 gives the calculated amounts of each polypeptide found in the PBS for Synechococcus sp 7002 based on this work.

A summary of the published spectral properties of each trimer found in the core is given in Table 1-2. The trimers containing $\mathrm{L}_{\mathrm{cm}}$, $\alpha^{\mathrm{AP}-\mathrm{B}}$, and $\beta^{18}$ have emission maxima at $678 \mathrm{~nm}$ while a simple AP 


\section{Table 1-1}

Composition of Synechococcus sp. PCC 7002 Phycobilisome*

Polypeptide

$\begin{array}{ll}\alpha^{\mathrm{PC}} & 72 \\ \beta^{\mathrm{PC}} & 72 \\ \mathrm{~L}_{\mathrm{r}}{ }^{33} & 6 \\ \mathrm{~L}_{\mathbf{r}}{ }^{8.5} & 6 \\ \mathrm{~L}_{\mathrm{r}}{ }^{29} & 6 \\ & \\ \alpha^{\mathrm{AP}} & 32 \\ \beta^{\mathrm{AP}} & 34 \\ \alpha^{\mathrm{AP}-\mathrm{B}} & 2 \\ \beta^{18} & 2 \\ \mathrm{~L}_{\mathrm{c}}^{8} & 6 \\ \mathrm{~L}_{\mathrm{cm}}{ }^{97} & 2\end{array}$

Copies/PBS

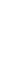




\section{Table 1-2}

Emission Maxima of Isolated Phycobilisome Core Trimers* Complex Copies/PBS

$$
F_{\max }
$$

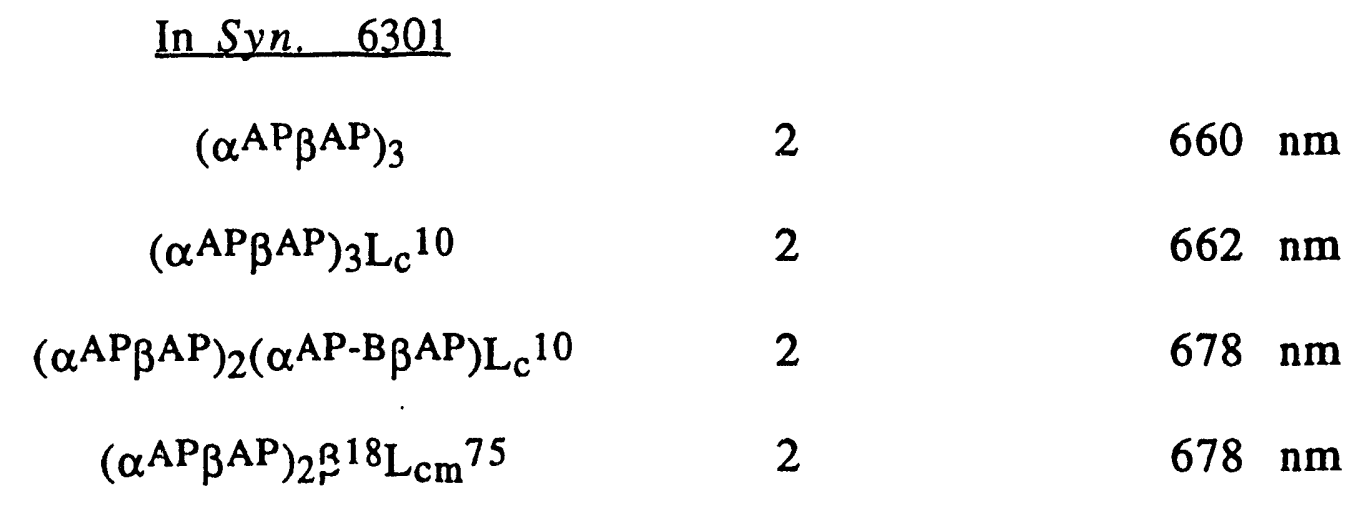

In Syn. 7002

$\left(\alpha^{\mathrm{AP}} \beta \mathrm{AP}_{3}\right.$

4

$660 \mathrm{~nm}$

$\left(\alpha{ }^{A P} \beta^{A P}\right)_{3} L_{c}^{8}$

4

$662 \mathrm{~nm}$

$\left(\alpha^{\mathrm{AP}} \beta \mathrm{AP}\right)_{2}\left(\alpha^{\mathrm{AP}-\mathrm{B}} \beta^{\mathrm{AP}}\right) \mathrm{L}_{c}{ }^{8}$

2

$678 \mathrm{~nm}$

$(\alpha \mathrm{AP} \beta \mathrm{AP})_{2} \beta^{18} \mathrm{~L}_{\mathrm{cm}} 97$

2

$678 \mathrm{~nm}$

* From J.C. Gingrich, D.J. Lundell, and A.N. Glazer, 1983 
trimer emits at $660 \mathrm{~nm}$. The $\beta^{18}$ and $\mathrm{L}_{\mathrm{cm}}$ polypeptides are found in the same trimer, so it is difficult to separate the contributions ofeither chromoprotein to the absorption and emission spectrum of the trimer.

The arrangement of the proteins in the core has been studied by several groups. Glazer's laboratory was successful in developing a relatively stable preparation, the $18 \mathrm{~S}$ preparation, containing only part of the core (Yamanaka, Lundell et al.,1982; Lundell and Glazer, 1983b) In the $18 \mathrm{~S}$ preparation they were able to say that the $\left(\alpha^{\mathrm{AP}} \beta{ }^{\mathrm{AP}}\right)_{3}$ and the $\left(\alpha^{\mathrm{AP}} \beta^{\mathrm{AP}}\right)_{2} \beta^{18} \mathrm{~L}_{\mathrm{cm}}$ trimers were adjacent in the core. Work carried out by other laboratories indicates that trimers containing the small linker polypeptide should be located on the ends of the core cylinders. This includes dissociation studies by Reuter and Wehrmeyer (Reuter and Wehrmeyer, 1988) and Anderson and Eiserling (Anderson and Eiserling, 1986). In addition, de Lorimier and Bryant at Pennsylvania State University were able to show that the $8 \mathrm{kD}$ polypeptide found in core trimers $\mathrm{A}$ and $\mathrm{D}$ shown in Figure 1-3 is structurally related to a $8.5 \mathrm{kD}$ protein found capping the rods (Bryant, 1988; de Lorimier, Bryant et al., 1990). Using this information, the only allowed arrangement for the apical core cylinder is shown in Figure 1-3 with the two $\left(\alpha^{\mathrm{AP}} \beta \mathrm{AP}\right)_{3}$ trimers in the interior on the core and the $\left(\alpha^{A P} \beta_{\beta} A P\right)_{3} L_{c} 8$ on the outside.

The cylinders found adjacent to the membrane could be arranged in two different ways. First, the trimers containing the small capping linker (trimers $A$ and $D$ in Figure 1-3) need to be on 
the outside. This would make the trimers containing $(\alpha \mathrm{AP} \beta \mathrm{AP})_{3}$ (trimer $\mathrm{C}$ ) and $(\alpha \mathrm{AP} \beta A \mathrm{P})_{2} \beta^{18} \mathrm{~L}_{\mathrm{cm}}$ (trimer $\mathrm{B}$ ) adjacent as required, but is trimer $A$ adjacent to trimer $B$ or trimer $C$ ? The two arrangements are shown in Figure 1-3.

One other thing to consider is the arrangement of the bottom cylinders relative to each other. Are they arranged in a parallel or anti-parallel fashion? The anti-parallel arrangement is preferred, based on the arrangement of the $\mathrm{L}_{\mathrm{cm}}$ polypeptide, and its efficiency in binding the core together. It has been proposed that the $\mathrm{L}_{\mathrm{cm}}$ polypeptide acts in binding the core trimers together (Bryant, 1988; Capuano, Braux et al., 1991). If the basal cylinders are arranged in an anti-parallel fashion, one $L_{c m}$ protein will be present in each half of the core, and the $\mathrm{L}_{\mathrm{cm}}$ should be able to bind that half of the core trimers together in an efficient manner. In the parallel arrangement both $\mathrm{L}_{\mathrm{cm}}$ polypeptides would be present in one-half of the core. This makes the binding of the core trimers in the other half of the core more difficult to envision. The $\mathrm{L}_{\mathrm{cm}}$ polypeptide would need to have binding domains the entire length of the core making the parallel arrangement more inefficient. The schematic in Figure 1-3 gives the preferred anti-parallel arrangement.

The functions of the unusual polypeptides ( $\alpha^{A P}-B, \beta^{18}$, and $L_{c m}$ ) found in the core are somewhat ill-defined at this time. I will describe their isolation along with their physical and spectral properties in the following paragraphs. 
The $\beta 18$ subunit

The $\beta^{18}$ was the last core polypeptide to be identified; it was isolated from Synechococcus 6301 AN112 using two dimensional mapping (SDS-polyacrylamide electrophoresis followed by isoelectric focusing) (Yamanaka, Lundell et al., 1982). It had never been identified before because the molecular weight of the polypeptide is very similar to that of $\alpha^{\mathrm{AP}}(18.3 \mathrm{kD}$ versus $18.2 \mathrm{kD}$ ), and it ran with the $\alpha^{\mathrm{AP}}$ band on SDS-PAGE. The $\beta^{18}$ polypeptide from Synechococcus 6301 was found to carry one phycocyanobilin chromophore, and it was judged to be present in roughly the same concentration as $\alpha$ AP-B.

The $\beta^{18}$ subunit was shown to be in the same core trimer as the large linker polypeptide in the $18 \mathrm{~S}$ core preparation (Lundell and Glazer,1983b). Using ${ }^{14}$ C-labeled PBS from Synechococcus 6301, Lundell and Glazer found that the trimer contained the ratio of $\beta^{18}$ : large linker polypeptide: $\beta \mathrm{AP}: \alpha \mathrm{AP}$ of $1: 1: 2: 2$ giving a core trimer of $\left(\beta^{A P} \alpha^{A P}\right)_{2} \beta^{18} L^{75}$. They were successful in isolating the $\beta^{18}$ subunit from the other polypeptides using preparative isoelectric focusing. The absorption spectrum of the purified subunit closely resembles that of isolated AP subunits with an $\lambda_{\text {abs }}$ of $620 \mathrm{~nm}$. Lundell and Glazer attempted to form AP trimers containing the subunit, but the $\beta^{18}$ subunit appeared to inhibit the formation of trimers. Based on this evidence they postulated that the $\beta^{18}$ subunit somehow mediates the interaction between the large linker polypeptide and the AP subunits in the trimer. 
The $\beta^{18}$ subunit has also been isolated from a few other species including Mastigocladus laminosus (Füglistaller, Rümbeli et al., 1984; Rümbeli, Wirth et al., 1987) and Synechococcus sn 7002 (Bryant, de Lorimier et al., 1990). Füglistaller sequenced the $M$. laminosus subunit and reported that the sequence homology of $\beta^{18}$ resembles that of the $\beta^{A P}$ subunit for amino acids $1-138(62 \%$ homology), but the final amino acids from 139 - 171 show very little homology with $\beta A P$. Bryant reported that the absorption spectrum of $\left(\alpha^{A P} \beta^{18}\right)_{1-3}$ is slightly red-shifted from that of the AP trimer. It is difficult to predict how the $\beta^{18}$ spectral properties will be affected upon its incorporation into a trimer with normal AP and $\mathrm{L}_{\mathrm{cm}}$.

\section{The $\alpha$ AP-B subunit}

The $\alpha$ AP-B subunit was first isolated using column chromatography in 1975 by Glazer and Bryant (Glazer and Bryant,1975; Ley, Butler et al., 1977). It was immediately identified as a likely terminal emitter in the PBS core, because iis spectral properties were red-shifted from those of AP. The polypeptide was originally isolated in a ( $\alpha \mathrm{AP}-\mathrm{B} \beta \mathrm{AP})$ trimer, but further work has shown that the substitution of only one $\alpha^{\mathrm{AP}}$ subunit with $\alpha^{\mathrm{AP}-\mathrm{B}}$ is required to give the trimer red-shifted spectral properties (Lundell and Glazer, 1981). AP trimers containing one $\alpha^{\mathrm{AP}-\mathrm{B}}$ subunit fluoresce at 678 $\mathrm{nm}$, versus $660 \mathrm{~nm}$ for a simple AP trimer, with approximately $85 \%$ of the emission coming from the $\alpha^{\text {AP-B }}$ subunit. Only two copies per 
PBS of the subunit were found in Synechococcus 6301 AN112 (Lundell and Glazer, 1983c).

The $\alpha$ AP-B subunit was found to be somewhat smaller than $\alpha^{\mathrm{AP}}$ $(17,800 \mathrm{~d}$ versus $18,200 \mathrm{~d}$ in Syn 6301) with a more basic isoelectric point. Isolated $\alpha$ AP-B also has altered absorption and fluorescence spectra compared to $\alpha^{A P}$ with $\lambda_{a b s}$ of $645-650 \mathrm{~nm}$ and $\lambda_{F}$ of $672 \mathrm{~nm}$, while the $\alpha^{A P}$ subunit maxima are at $617 \mathrm{~nm}$ and $642 \mathrm{~nm}$ respectively. (Lundell and Glazer, 1981; Lundell and Glazer, 1983c; Yamanaka, Lundell et al., 1982). The quantum yield of fluorescence of the isolated $\alpha$ AP-B subunit was also found to be much smaller than that of the isolated $\alpha \mathrm{AP}$ subunit, 0.05 versus 0.2 , even though the same chromophore is thought to be in both proteins (Lundell and Glazer, 1981). The $\alpha^{A P-B}$ subunit also has been isolated from other ol ganisms including Mastigocladus laminosus (Suter, Füglisialler et al., 1987) and Rhodella violacea (Reuter, Nickel et al., 1950). There appears to be a great deal of similarity in the amino acid sequence and spectral characteristics of the subunits from the various organisms.

\section{The $L_{c m}$ Polypeptide}

The $\mathrm{L}_{\mathrm{cm}}$ protein is probably the most interesting polypeptide found in the PBS core, but it also has proved to be the most difficult to study since it is very vulnerable to proteolysis (Yamanaka, Lundell et al., 1982; Gingrich, Lundell et al., 1983). The protein was first isolated with chromophore intact in 1981 by Redlinger and Gantt (Redlinger ar.' Gantt, 1981). It was previously believed 
to be colorless since the techniques used in the isolation somehow bleached the chromophore. This protein is also believed to contain the same phycocyanobilin chromophore found in AP and PC (Lundell, Yamanaka et al., 1981).

The $\mathrm{L}_{\mathrm{cm}}$ polypeptide was successfully isolated from other proteins in the core using a denaturing technique, SDS-PAGE. The protein can be renatured to give an $\lambda_{\mathrm{abs}}=665 \mathrm{~nm}$ and $\lambda_{\mathrm{F}}=676 \mathrm{~nm}$ (Lundell, Yamanaka et al., 1981). The integrity of the renatured protein was questioned, because the quantum yield was found to be 0.16 , lower than that of most phycobiliproteins. Work using core fractionation methods has shown that an isolated trimer of $\left(\alpha^{A P_{\beta} A P}\right)_{2} \beta^{18} L_{c m}$ has a $\lambda_{F}=678 \mathrm{~nm}$, but it is difficult to separate out the individual effects of $L_{c m}$ and $\beta^{18}$ within the trimer.

The role of the $\mathrm{L}_{\mathrm{cm}}$ polypeptide must be more than simply that of terminal emitter in the core since it is about $100 \mathrm{kD}$ in size. Originally, the protein was believed to anchor the PBS complex to the photosynthetic membrane; now it appears that the large polypeptide in the core participates in the binding of the rods to the core. I will try to bring together a lot of data on the role of the $L_{c m}$ polypeptide in the next few paragraphs.

A very complete study was carried out on the $L_{c m}$ protein found in Anabaena variabilis by Isono and Katoh (Isono and Katoh, 1987). Using a variety of methods they were able to show that very little of the large linker polypeptide is accessible to solvent in intact PBS. First, they tried binding fluorescence probes to intact PBS or intact cores. The probes bound minimally to the linker polypeptide in the intact systems but showed enhanced binding to the $\mathrm{L}_{\mathrm{cm}}$ in 
dissociated PBS. Second, they took advantage of the preferential digestion of $\mathrm{L}_{\mathrm{cm}}$ with chymotrypsin. In intact PBS the large polypeptide was reduced in molecular weight as judged by SDSPage from $115 \mathrm{kD}$ to $105 \mathrm{kD}$, while for intact core it went from 115 $\mathrm{kD}$ to $84 \mathrm{kD}$. Isono and Katoh also digested a subcore preparation called a $14.5 \mathrm{~S}$ APC preparation in which $\mathrm{L}_{\mathrm{cm}}$ was reduced in molecular weight from $115 \mathrm{kD}$ to $42 \mathrm{kD}$ over a thirty minute incubation with chymotrypsin. In none of the digestions did the "far-red fluorescence" spectral properties alter. Isono and Katoh also carried out antibody studies in which antibodies raised against $\mathrm{L}_{\mathrm{cm}}$ did not react with intact PBS or core but did react with the 14.5 $S$ subcore preparation. They concluded that most of the polypeptide is embedded inside the PBS core with parts becoming exposed upon the loss of rods. They proposed that the large linker polypeptide is involved in the binding of AP trimers within the core.

Based on their chymotrypsin digestion studies of the polypeptide, Isono and Katoh proposed that the polypeptide from Anabaena variabilis is composed of four domains of $10,42,42$, and $21 \mathrm{kD}$ respectively. The $10 \mathrm{kD}$ domain which is removed from intact PBS possibly is the part of the polypeptide that binds the PBS to the photosynthetic membrane. The second domain $(42 \mathrm{kD})$ is the part of the polypeptide that binds the $14.5 \mathrm{~S}$ subcore preparation together and contains the chromophore. The third domain (42 kD) is involved in binding the rest of the core while the fourth domain $(21 \mathrm{kD})$ binds the rods to the core. 
Better understanding of the role of $\mathrm{L}_{\mathrm{cm}}$ came with the sequencing of the polypeptide. In recent years much work has been done in determining the amino acid sequences of the $\mathrm{L}_{\mathrm{cm}}$ polypeptide in different organisms. The sequence has been determined for Calothrix sp PCC 7601 (Houmard, Capuano et al., 1990), Synechococcus 7002 (Bryant, 1991) and Synechococcus 6301 (Capuano, Braux et al., 1991). For all of the proteins studied the sequences can be separated into three types of domains: linker, phycobiliprotein, and lengths of amino acids with no homology connecting the linker and phycobiliprotein domains. For example, in Calothrix sp PCC 7601 the $\mathrm{L}_{\mathrm{cm}}$ polypeptide contains one domain from amino acids 18-237 that is homologous to a phycobiliprotein subunit, especially in terms of the binding pocket. Four other sequence domains are very similar to rod linker proteins, while the segments connecting the different domains have no known homology and are of differing lengths. Similar conclusions were made with the sequences from Synechococcus 7002 and Synechococcus 6301, although the number of sequences homologous with rod linker polypeptides differs with the type of phycobilisome core found in that species. The $\mathrm{L}_{\mathrm{cm}}$ can be described as being composed of a phycobiliprotein domain starting at the $\mathrm{N}$ terminal end followed by two, three, or four linker domains. The number of linker domains appears to correlate with the number of cylinders contained in the PBS core (Capuano, Braux et al., 1991).

Based on this information it appears that the large polypeptide found in the core plays a dual role in both binding the rod and core cylinders together and accepting energy from chromophores in the 
core for transfer to chl $a$ pigments. One could also speculate that the large linker polypeptide might also hold the PBS onto the thylakoid membrane, but no transmembrane sequences characteristic of membrane binding have yet been identified in the sequenced proteins.

\section{The Project}

To further elucidate the roles played by the unusual polypeptides in the core $\left(\alpha A P-B, \beta^{18}\right.$, and $\left.L_{c m}\right)$ we studied some mutants that were produced in Bryant's laboratory at Pennsylvania State University (Zhou, 1991). Bryant has been successful in cloning, sequencing, and characterizing all 11 polypeptides found in Synenchococcus 7002 PBS (Bryant, 1991). In Bryant's laboratory several different core mutations of Synechococcus 7002 were created using both interposon mutagenesis and/or point mutations.

The mutants I studied included the apcF mutant which is missing $\beta^{18}$, the apcD mutant missing $\alpha^{\mathrm{AP}-\mathrm{B}}$, the double mutant of apcDF, the apcE/C186S mutant which is missing the chromophorebinding cysteine at position 186 , and the double mutant apcDE/C186S. The apcE/C186S and apcDE/C186S mutants proved to be especially interesting because they contain a modified chromophore on $\mathrm{L}_{\mathrm{cm}}$ instead of losing the chromophore entirely.

Earlier work was done on the apcD mutant by Maxson in this laboratory (Maxson, Sauer et al., 1989); I have reproduced some of that work along with expanding the study on the apcD mutant. I have a relatively complete set of data on all of the mutants including steady state absorption and emission and time-resolved 
emission on whole cells, intact PBS, and isolated $18 \mathrm{~S}$ preparations; I am only missing the time-resolved emission data on the apcDE/C186S $18 \mathrm{~S}$ preparation.

The deletion mutants of $a p c D, a p c F$ and $a p c D F$ were created by interrupting the gene of interest with an antibiotic resistance marker using recombinant DNA methods (Bryant, 1988). First, the gene had to be cloned, sequenced, and identified. Next, the DNA for that gene was expressed in $E$. coli, and the gene was interrupted or replaced using a sequence of DNA that encodes for antibiotic resistance using homologous recombination DNA techniques. (Golden, Brussian et al., 1987). The altered DNA was then added back into the cyanobacterium, which is transformable by exogenously added homologous DNA; Synenchococcus. 7002 is a strain of cyanobacteria that can incorporate foreign DNA with homologous sequences into its own DNA. The cells with the altered DNA were selected for by antibiotic resistance. DNA from the selected cells was then sequenced to ensure that the antibiotic resistance marker had been incorporated into the DNA at the correct position.

In the following chapters I will discuss the spectral properties of the whole cells, intact PBS, and core preparations from the mutant and wild-type strains. From these data I will try to assign spectral maxima to the polypeptides, and identify their roles in the antenna complexes. 
References for Chapter 1

Anderson, L. K. and F. A. Eiserling (1986). J. Mol. Biol., 191, 441-451.

Braslavsky, S. E., A. R. Holzwarth, and K. Schaffner (1983). Angew. Chem. Int. Ed. Engl., 22, 656-674.

Bruce, D., S. Brimble, and D. A. Bryant (1989). Biochim. Biophys. Acta, 974, 66-73.

Bryant, D. A. (1988). Genetic analysis of phycobilisome biosynthesis, assembly, structure, and function in the cyanobacterium Synechococcus sp. PCC 7002, In S. E. Stevens, Jr., and D. A. Bryant (eds.) Light-Energy Transduction in Photosynthesis: Higher Plant and Bacterial Models. The American Society of Plant Physiologists, Rockville, MD (USA) 62-93.

Bryant, D. A., R. de Lorimier, G. Guglielmi, and S. E. Stevens, Jr. (1990). Arch. Microbiol., 153, 550-560.

Bryant, D. A. (1991). Cyanobacterial phycobilisomes: progress toward complete structural and functional analysis via molecular genetics. In L. Bogorad \& I. K. Vasil (eds.), Cell Culture and Somatic Cell Genetics of Plants., Vol 7B, Academic Press, Inc., London 257300.

Capuano, V., A. Braux, N. Tandeau de Marsac, and J. Houmard (1991). J. Biol. Chem., 266, 7239-7247.

Clement-Metral, J. D. and E. Gantt (1983). FEBS Lett., 156, 185-92.

de Lorimer, R., G. Giglielmi, D. A. Bryant, and S. E. Stevens, Jr. (1990). Arch. Microbiol., 153, 541-549.

de Lorimer, R., D. A. Bryant, and S. E. Stevens, Jr. (1990). Bichim. Biophys. Acta, 1019, 29-41.

Deisenhofer, J., O. Epp, K. Miki, R. Huber, and H. Michel (1984). J. Mol. Biol., 180, 385-398.

Dilworth, M. F. and E. Gantt (1981). Plant Physiol., 67, 608-12. 
Füglistaller, P.,R. Rümbeli, F. Suter, and H. Zuber (1984). HoppeSeyler Z Physiol Chem, 365, 1085-1096.

Füglistaller, P., M. Mimuro, F. Suter, and H. Zuber (1987). Biol. Chem. Hoppe-Seyler, 368, 353-367.

Gantt, E. (1980). Int. Rev. Cytol., 66, 45-80.

Gantt, E. (1981). Ann. Rev. Plant Physiol., 32, 327-47.

Gingrich, J. C., D. J. Lundell, and A. N. Glazer (1983). J. Cell. Biochem., $22,1-14$.

Glauser, M., W. Sidler, and H. Zuber (1993). Photochem. Photobiol., 57: 344-351.

Glazer, A. N. and D. A. Bryant (1975). Arch. Microbiol., 104, 15-22.

Glazer, A. N., R. C. Williams, G. Yamanaka, and H. K. Schachman (1979). Proc. Natl. Acad. Sci. USA, 76, 162-166.

Glazer, A. N. (1983). Ann. Rev. Biochem., 52, 125-57.

Glazer, A. N. (1984). Biochim. Biophys. Acta, 768, 29-51.

Glazer, A. N. (1985). Ann. Rev. Biophys. Chem., 14, 47-77.

Glazer, A. N. (1989). J. Biol. Chem., 264, 1-4.

Golden, S. S., J. Brusslan, and R. Haselkorn (1987). Genetic engineering of the cyanobacterial chromosome, In R. Wu and $\mathrm{L}$. Grossman (eds.), Methods in Enzymology., Vol 153, Academic Press, Inc., New York 215-231.

Guard-Friar, D. and R. MacColl, (1984). Arch. Biochem. Biophys., 230, 300-305.

Houmard, J., V. Capuano, M. V. Colombano, T. Coursin, and N. Tandeau de Marsac (1990). Proc. Natl. Acad. Sci. USA, 87, 21522156. 
Isono, T. and T. Katoh (1987). Arch. Biochem. Biophys., 256, 317324.

Katoh, T. and E. Gantt (1979). Biochim. Biophys. Acta, 546, 383-393.

Kura-Hotta, M., K. Satoh, and S. Katoh (1986). Arch. Biochem. Biophys., 249, 1-7.

Ley, A. C.,W. L. Butler, and D. A. Bryant (1977). Plant Physiol., 59, 974-80.

Lundell, D. J., R. C. Williams, and A. N. Glazer (1981). J. Biol. Chem., $256,3580-3592$.

Lundell, D. J., G. Yamanaka, and A. N. Glazer (1981). J. Cell. Biol., 91, 315-319.

Lundell, D. J. and A. N. Glazer (1981). J. Biol. Chem., 256, 1260012606.

Lundell, D. J and A. N. Glazer (1983a). J. Biol. Chem., 258, 87088713.

Lundell, D. J. and A. N. Glazer (1983b). J. Biol. Chem., 258, 894-901.

Lundell, D. J. and A. N. Glazer (1983c). J. Biol. Chem., 258, 902-908.

Manodori, A. and A. Melis (1985). FEBS Lett., 181, 79-82.

Maxson, P., Sauer, K., J. Zhou, D. A. Bryant, and A. N. Glazer (1989). Biochim. Biophys. Acta 977: 40-51.

Mullineaux, C. and A. R. Holzwarth (1990). FEBS Lett., 260, 245-248.

Mullineaux, C. W. (1992). Biochim. Biophys. Acta, 1100, 285-292.

Porter, G., C. J. Tredwell, G. R. W. Searle, and J. Barber (1978). Biochim. Biophys. Acta, 501, 232-245.

Redlinger, T. and E. Gantt (1981). Plant Physiol., 68, 1375-1379. 
Reuter, W. and W. Wehrmeyer (1988). Arch. Microbiol., 150, 534540.

Reuter, W., C. Nickel, and W. Wehrmeyer (1990). FEBS Lett., 273, $155-158$.

Rümbeli, R., M. Wirth, F. Suter, and H. Zuber (1987). Biol. Chem. Hoppe-Seyler, 368, 1-9.

Scheer, H. (1986). Excitation Transfer in Phycobiliproteins, In L. A. Staehelin, and C. J. Arntzen (eds), Encyclopedia of Plant Physiology., Vol 19, Springer-Verlag, Berlin 327-337.

Schirmer, T., W. Bode, and R. Huber (1987). J. Mol. Biol., 196, 677695.

Stevens, S. E., D. L. Balkwill, and D. A. M. Paone (1981). Arch. Microbiol., 130, 204-212.

Suter, F., P. Füglistaller, D. J. Lundell, A N. Glazer, and H. Zuber (1987). FEBS Lett., 217, 279-282.

Tandeau de Marsac, N. and G. Cohen-Bazire (1977). Proc. Natl. Acad. Sci. USA, 74, 1635-1639.

Witt, H. T., N. Krauss, W. Hinrichs, I. Witt, P. Fromme, and W. Saenger (1992). Three-dimensional crystals of photosystem I from Synechococcus $s p$. and X-ray structure analysis at $6 \AA$ resolution, In N. Murata (ed.), Research in Photosynthesis. IXth International Congress on Photosynthesis. Vol 1, Kluwer Academic Publishers, Dordrecht, 521-528.

Yamanaka, G., A. N. Glazer and R. C. Williams (1978). J. Biol. Chem., $253,8303-8310$.

Yamanaka, G., D. J. Lundell, and A. N. Glazer (1982). J. Biol. Chem., $257,4077-4086$.

Yu, M. H. and A. N. Glazer (1982). J. Biol. Chem., 257, 3429-3433.

Zhou, J. (1991). Ph. D. Thesis, Pennsylvania State University. 


\section{Chapter 2. Materials and Methods}

\section{The Cultures}

The wild-type and mutant strains of Synechococcus sp. PCC 7002 were obtained from D.A. Bryant's laboratory at Pennsylvania State University (Zhou, 1991). The cultures were grown using Medium At (see Table 2-1) with $200 \mu \mathrm{E} \mathrm{m}^{-2} \mathrm{~s}^{-1}$ of white fluorescent light at approximately $32{ }^{\circ} \mathrm{C}$. The cultures were aerated with an approximate $5 \%(\mathrm{v} / \mathrm{v}) \quad \mathrm{CO}_{2} / \mathrm{N}_{2}$ gas mixture with constant stirring. Doubling times for the various strains are given in Table 2-2 (Bryant, personal communication).

Continuous stock cultures used to provide starting stock were grown in $125 \mathrm{ml}$ Erlenmeyer flasks under the same temperature and light conditions as above but without $\mathrm{CO}_{2}(\mathrm{~g})$. The stock cultures were grown with the antibiotics listed in Table 2-1 to prevent reversion to wild-type while the larger cultures were generally grown without the antibiotics. The large cultures were harvested during the end of the log phase of growth which generally was 7 to 10 days for a 1.51 culture.

\section{Preparation of Intact Phycobilisomes}

The preparation for intact PBS was taken from Maxson's thesis with slight modifications (Maxson, 1988) and is described in the following paragraphs. Cells near the end of the log phase of growth were concentrated by centrifugation in a Dupont GS-3 Rotor for $5 \mathrm{~min}$ at $8500 \mathrm{rpm}$. The cells were resuspended in growth 
Table $2-1$

Medium A+ Recipe

\begin{tabular}{|c|c|c|c|}
\hline Chemical & $\begin{array}{l}\text { Amount } \\
(\mathrm{g} / \mathrm{l}) \mathrm{a}\end{array}$ & $\begin{array}{l}\text { Concentration } \\
\text { of Stock }(\mathrm{g} / \mathrm{l}) \mathrm{a}\end{array}$ & $\begin{array}{l}\text { Volume }(\mathrm{ml}) \text { of } \\
\text { Stock per } 1 \text { liter } \\
\text { of Medium }\end{array}$ \\
\hline $\mathrm{NaNO}_{3}$ & 1.0 & 500 & 2.0 \\
\hline $\mathrm{NH}_{4} \mathrm{Cl}$ & 0.15 & 150 & 1 \\
\hline $\mathrm{K}_{2} \mathrm{HPO}_{4} \cdot 3 \mathrm{H}_{2} \mathrm{O}$ & 0.050 & 50 & 1 \\
\hline $\mathrm{CaCl}_{2} \cdot 2 \mathrm{H}_{2} \mathrm{O}$ & 0.23 & 230 & 1 \\
\hline Salt Solution & & & 100 \\
\hline $\mathrm{MgSO}_{4}$ & 2.44 & 24.4 & \\
\hline $\mathrm{NaCl}$ & 18 & 180 & \\
\hline $\mathrm{Ka}$ & 0.6 & 6 & \\
\hline $\mathrm{Na}_{2} \mathrm{EDTA}^{\mathrm{b}}$ & 0.030 & 30 & 1 \\
\hline $1.0 \mathrm{M}$ Tris, $\mathrm{pH} 8$ & $8.5 \mathrm{mM}$ & $1.0 \mathrm{M}$ & 8.5 \\
\hline $\mathrm{FeCl}_{3} \cdot 6 \mathrm{H}_{2} \mathrm{O}$ & $4.0 \mathrm{mg} / 1$ & 4.0 & 1 \\
\hline Trace Metals & & & 1 \\
\hline $\mathrm{H}_{3} \mathrm{BO}_{3}$ & $34.2 \mathrm{mg} / 1$ & 34.2 & \\
\hline $\mathrm{MnCl}_{2} \cdot 4 \mathrm{H}_{2} \mathrm{O}$ & $4.3 \mathrm{mg} / 1$ & 4.3 & \\
\hline $\mathrm{ZnSO}_{4} \cdot 7 \mathrm{H}_{2} \mathrm{O}$ & $0.63 \mathrm{mg} / \mathrm{l}$ & 0.63 & \\
\hline $\mathrm{Na}_{2} \mathrm{MoO}_{4} \cdot 2 \mathrm{H}_{2} \mathrm{O}$ & $3.9 \mathrm{mg} / 1$ & 3.9 & \\
\hline $\mathrm{CuSO}_{4} \cdot \mathrm{SH}_{2} \mathrm{O}$ & $30 \mu g / 1$ & 0.03 & \\
\hline $\mathrm{CoCl}_{2} \cdot 6 \mathrm{H}_{2} \mathrm{O}$ & $12.2 \mu \mathrm{g} / 1$ & 0.0122 & \\
\hline Vitamin $B^{12}$ & $5 \mu g / 1$ & $50 \mathrm{mg} / \mathrm{l}$ & 0.1 \\
\hline \multicolumn{4}{|l|}{ Antibiotics } \\
\hline$\overline{\text { Kanamycinc }}$ & $100 \mathrm{mg} / \mathrm{l}$ & 80 & 1.25 \\
\hline Ampicillind & $5 \mathrm{mg} / 1$ & 5 & 1 \\
\hline Erythromycine & $20 \mathrm{mg} / 1$ & 10 & 2 \\
\hline
\end{tabular}

a amounts in $g / l$ unless specified otherwise

b Can substitute with $1 \mathrm{ml}$ of $0.1 \mathrm{M}$ EDTA pH 8

c Add Kanamycin to all mutant cultures

d Add Ampicillin to apcE/C186S and apcDE/C186S cultures

: Add Erythromycin to apcDF and apcDE/C186S cultures 
Table 2-2

Doubling Times of Mutants

\begin{tabular}{|c|c|c|}
\hline Strain & $\begin{array}{l}\text { High Light } \\
\text { Intensity }^{\mathrm{a}}\end{array}$ & $\begin{array}{l}\text { Low Light } \\
\text { Intensityb }\end{array}$ \\
\hline Wild-Type & $4.3 \mathrm{~h}$ & $11.1 \mathrm{~h}$ \\
\hline apcF & $5.1 \mathrm{~h}$ & $14.0 \mathrm{~h}$ \\
\hline apcDF & $5.4 \mathrm{~h}$ & $16.7 \mathrm{~h}$ \\
\hline apcD & $4.2 \mathrm{~h}$ & $11.4 \mathrm{~h}$ \\
\hline apcE/C186S & $20.4 \mathrm{~h}$ & $57.6 \mathrm{~h}$ \\
\hline
\end{tabular}


medium and centrifuged at $12000 \mathrm{rpm}$ for $5 \mathrm{~min}$ in a Dupont SS-34 Rotor to determine an accurate wet weight of cells. The cells were resuspended to $0.12 \mathrm{~g} \mathrm{ml}^{-1}$ with $0.75 \mathrm{M} \mathrm{NaKHPO}_{4} \mathrm{pH} 7.8$ (Buffer A) containing $1 \mathrm{mM} \mathrm{NaN} 3$ and approximately $20 \mu \mathrm{M}$ phenylmethylsulfonyl fluoride (PMSF, Sigma). The PMSF should be added just prior to using, because it degrades rapidly in water-based solvents.

The suspension was homogenized to remove large clumps of cells and then French pressed four times at $1.4 \mathrm{kbar}(20,000 \mathrm{psi})$ at $4{ }^{\circ} \mathrm{C}$. The ruptured cells were immediately incubated with $1.2 \%$ $(w / w)$ Triton $\mathrm{X}-100$ for $30 \mathrm{~min}$ at room temperature with vigorous stirring; the detergent concentration is critical to the quality of the preparation obtained. Unbroken cells and large cell fragments were removed by centrifuging for $30 \mathrm{~min}$ at $16,000 \mathrm{rpm}$ in a Dupont SS34 rotor at room temperature.

The suspension separated into three phases upon centrifugation; unbroken cells and large cell membranes pelleted to the bottom of the tube, chlorophyll and detergent collected at the top of the tube, and the phycobilisomes were found in the blue supernatant below the green chlorophyll detergent layer. The blue supernatant was carefully removed from the oiner components in the centrifuge tube and loaded onto a sucrose step gradient composed of $7.0 \mathrm{ml}$ each of $1.0 \mathrm{M}, 0.8 \mathrm{M}, 0.6 \mathrm{M}, 0.4 \mathrm{M}$, and $0.2 \mathrm{M}$ sucrose in Buffer A in a Beckman SW-28 or SW-27 centrifuge tube. The tubes were spun for $16 \mathrm{~h}$ at $21,000 \mathrm{rpm}$ and $15{ }^{\circ} \mathrm{C}$ with no brake in a Beckman ultracentrifuge. 
A typical sucrose gradient is shown in Figure 2-1 with the intact phycobilisomes found in the $0.6 \mathrm{M}$ sucrose layer. More free phycobiliprotein was found in the $0.4 \mathrm{M}$ sucrose layer for the mutants than for the wild-type preparations. The intact PBS were removed from the gradient after removing all of the upper bands to minimize contamination. All spectroscopy was carried out within $16 \mathrm{~h}$ after the protein was removed from the gradients.

\section{Preparation of the $18 \mathrm{~S}$ Core Particle}

The following procedure for preparation of the $18 \mathrm{~S}$ core preparation is a combination of several different procedures taken from Glazer's and Bryant's laboratories (Yamanaka, Lundell et al., 1982; Gingrich, Lundeil et al.,1983; Bryant, de Lorimier et al.,1990). Cells were harvested near the end of the log phase of growth and were concentrated using a Dupont GS-3 rotor for $5 \mathrm{~min}$ at $8500 \mathrm{rpm}$. The cells were resuspended using a minimum of growth medium and centrifuged a second time using a Dupont SS 34 rotor for 5 min at $12,000 \mathrm{rpm}$ to obtain an accurate cell wet weight. The cells were resuspended to $0.12 \mathrm{~g} \mathrm{ml}^{-1}$ in $50 \mathrm{mM}$ Tris buffer at pH 7.8 vith 0.8 $\mathrm{M} \mathrm{Na}_{2} \mathrm{SO}_{4}, 1 \mathrm{mM} \mathrm{NaN}$, and $20 \mu \mathrm{M}$ PMSF (Buffer B).

The cells were homogenized and French pressed four times at $1.4 \mathrm{kbar}(20,000 \mathrm{psi})$ and $4{ }^{\circ} \mathrm{C}$. The suspension was incubated with $1.2 \%(w / w)$ Triton $X-100$ for $30 \mathrm{~min}$ at room temperature with vigorous stirring. The suspension was then centrifuged for $30 \mathrm{~min}$ at $16,000 \mathrm{rpm}$ and $15^{\circ} \mathrm{C}$ in a Dupont SS-34 rotor. After centrifugation the suspension separated into three different components: a solid pellet composed of unbroken cells and cell membranes, a 


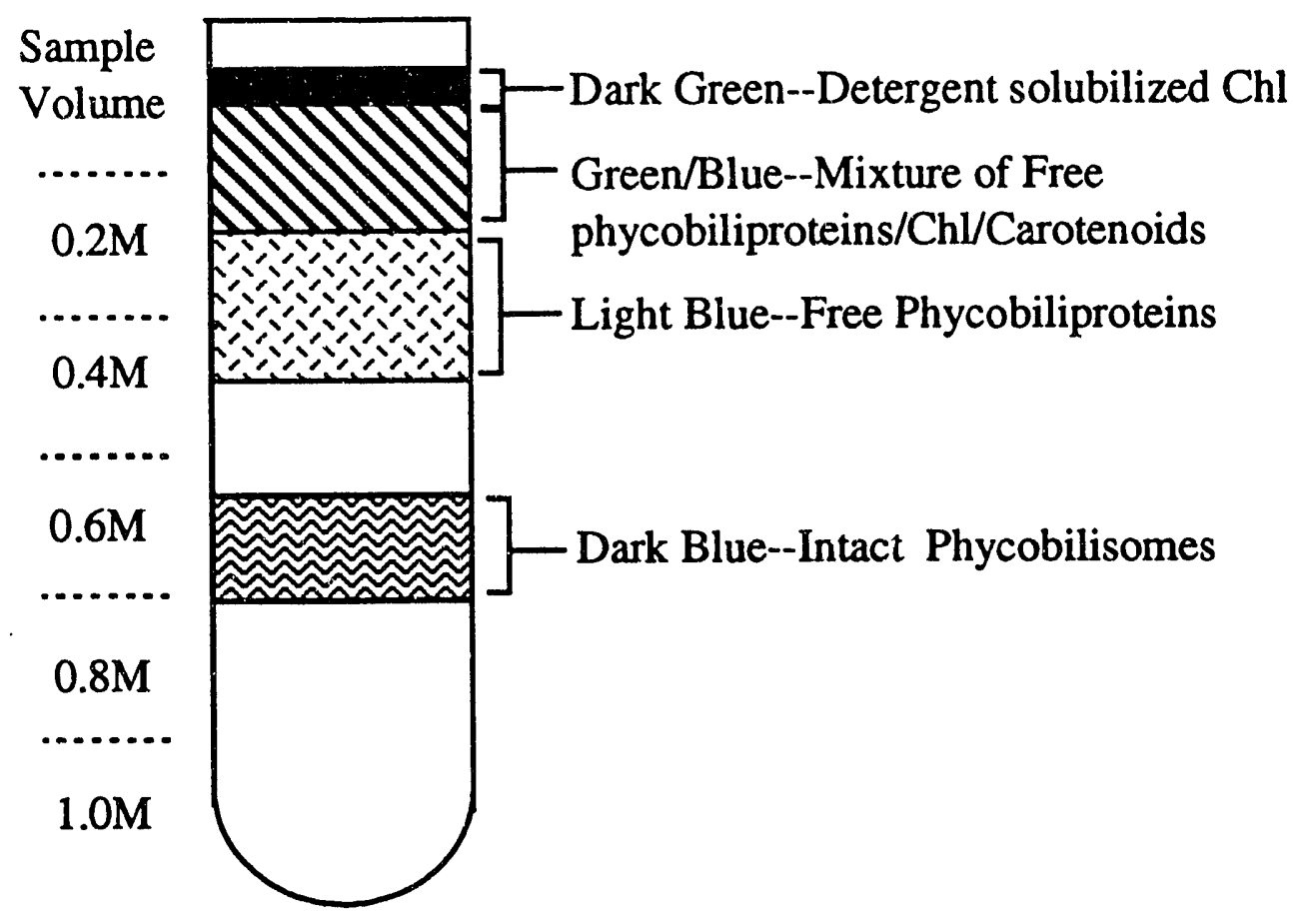

Figure 2-1. Sucrose Step Gradient from Phycobilisome Preparation.

Typical gradient profile in SW-27 tube. Intact PBS are located in the $0.6 \mathrm{M}$ sucrose layer. Free phycobiliproteins are found in the $\mathbf{0 . 4}$ and $0.2 \mathrm{M}$ sucrose. 
green top layer made up of chlorophyll and detergent, and a blue middle layer containing all of the phycobiliproteins. The blue supernatant was carefully removed from the gradient with special care to minimize contamination from the chlorophyll layer and was loaded onto a sucrose step gradient.

The sucrose step gradient was made up of $3 \mathrm{ml}$ of $2.0 \mathrm{M}, 5 \mathrm{ml}$ of $1.0 \mathrm{M}$, and $7 \mathrm{ml}$ each of $0.75 \mathrm{M}$ and $0.5 \mathrm{M}$ sucrose in Buffer $\mathrm{B}$. The gradients were centrifuged for $2-4 \mathrm{~h}$ in a Beckman 50.2Ti rotor at $45,000 \mathrm{rpm}$ and $15^{\circ} \mathrm{C}$ with no brake.

The gradient profile is shown in Figure 2-2a with the intact PBS found in the three dark blue bands found in the 0.75 and $1.0 \mathrm{M}$ sucrose layers. Using steady-state fluorescence and absorption spectroscopy no significant differences were found in the composition of the PBS within the three bands, so all of the bands were combined for the following steps. Significant amounts of chlorophyll were found in the $1.0 \mathrm{M}$ sucrose layer, but all the contaminating chlorophyll appeared to precipitate during the following steps.

After intact PBS were removed from the gradients they were exchanged into $50 \mathrm{mM}$ Tricine buffer at $\mathrm{pH} 7.8$ with $5 \mathrm{mM} \mathrm{CaCl}$, $10 \%$ (v/v) glycerol, $1 \mathrm{mM} \mathrm{NaN}_{3}$, and $20 \mu \mathrm{M}$ PMSF (buffer C) in which the intact PBS partially dissociated. The PBS could be exchanged into the Tricine buffer using either dialysis which takes $18 \mathrm{~h}$ or Bio-Rad 10-DG desalting columns (Richmond, CA) which takes about $2 \mathrm{~h}$. Next, the dissociated proteins were concentrated using an Amicon PM-30 Ultrafiltration membrane (Amicon 


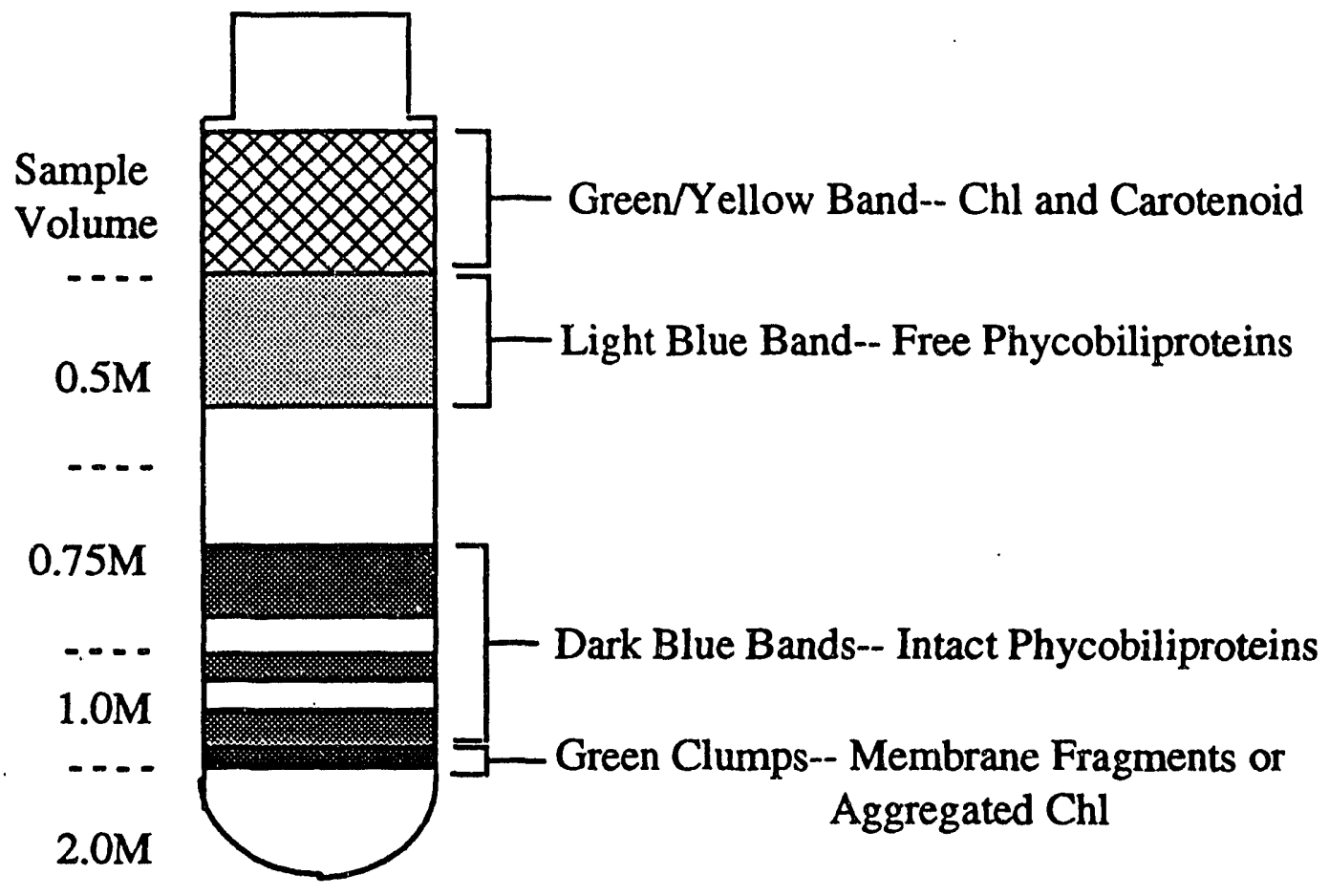

Figure 2-2a. Gradient containing Intact Phycobilisomes from $18 \mathrm{~S}$ Preparation.

Step gradient profile of $18 \mathrm{~S}$ preparation in SW-27 tube. Intact PBS are found in the 0.75 and $1.0 \mathrm{M}$ sucrose steps. Free phycobiliprotein is found in the $0.5 \mathrm{M}$ sucrose step. 
Company, Danvers, MA) at 3.7 bar (55 psi) of pressure until the absorption is $10-15 \mathrm{~cm}^{-1}$ at $630 \mathrm{~nm}$.

Approximately $3 \mathrm{ml}$ aliquots of the concentrated protein were loaded onto linear sucrose gradients composed of $0.9 \mathrm{M}$ to $0.3 \mathrm{M}$ sucrose in Buffer C. The gradients were centrifuged for $11-12 \mathrm{~h}$ at $41,000 \mathrm{rpm}$ in a Beckman 50.2Ti rotor at $15{ }^{\circ} \mathrm{C}$ with no brake. The gradient profile is shown in Figure $2-2 \mathrm{~b}$ with the $18 \mathrm{~S}$ core preparation located in the narrow, lower band. The upper broad band is composed mostly of PC hexamers with $\lambda_{\mathrm{abs}}=628 \mathrm{~nm}$ and $\lambda_{F}$ $=650 \mathrm{~nm}$. The $18 \mathrm{~S}$ core preparation was removed from the gradient using a syringe with a small gauge needle with extra care taken to avoid contamination with the upper broad band.

\section{HPLC of $18 \mathrm{~S}$ preparations}

To determine the composition of the $18 \mathrm{~S}$ preparations HPLC was carried out on wild-type and apcF samples using the protocol outlined in Swanson's paper (Swanson and Glazer, 1990). First, protein samples were prepared for HPLC by dialyzing the protein overnight against $5 \mathrm{mM}$ phosphate buffer, $\mathrm{pH} 7.0$ containing $1 \mathrm{mM} \mathrm{2-}$ mercapto-ethanol. The protein was then concentrated as much as possible using an Amicon PM-30 ultrafiltration membrane with $3.7 \mathrm{bar}$ ( $55 \mathrm{psi}$ ) of pressure, $4{ }^{\circ} \mathrm{C}$. Just prior to injection, the protein was diluted $1: 1$ with $9.0 \mathrm{M}$ urea $\mathrm{pH} 2.0$ and spun for $5 \mathrm{~min}$ in a microcentrifuge to remove any precipitates.

The HPLC chromatography was carried out using the Waters equipment (Waters Chromatography Division, Milford, MA) in A. Glazer's laboratory as described in Swanson's paper. The protein, 


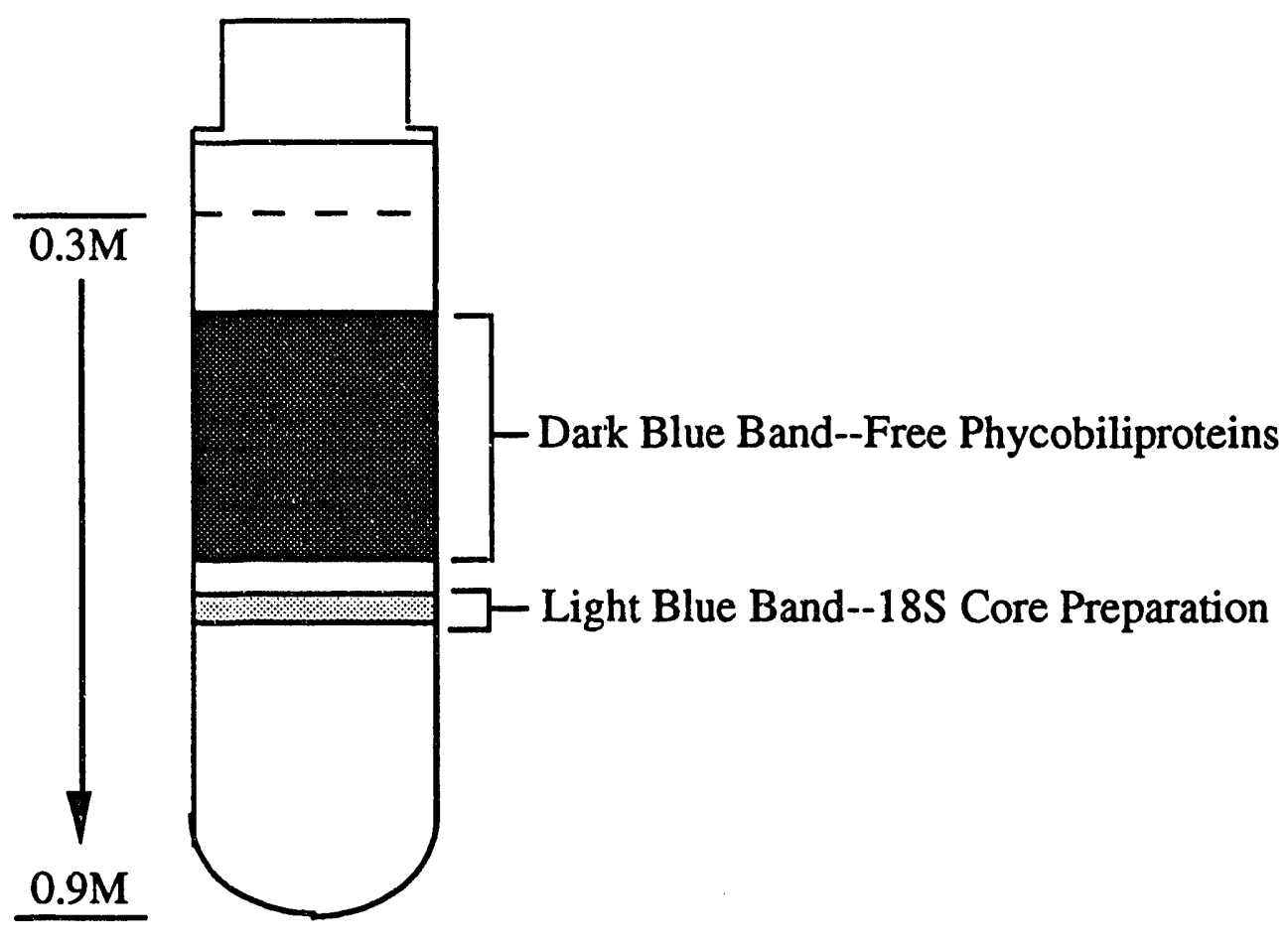

Figure 2-2b. Linear Sucrose Gradient of an $18 \mathrm{~S}$ Preparation.

Typical profile of the linear gradient shown in a 50.2Ti tube.

The $18 \mathrm{~S}$ preparation is located in the narrow, lower band. 
approximately $350 \mu \mathrm{l}$ in volume, is injected in a $\mathrm{C}_{4}$ analytical column ( Hipore RP-304, Bio-Rad, Richmond, CA) previously equilibrated with $65 \%$ water/ $35 \% 2: 1$ acetonitrile: isopropanol everything with $0.1 \%$ trifluoroacetic acid (TFA). The proteins were eluted from the column using the following protocol: $0-2 \mathrm{~min}, 65 \%$ water/ 35\% 2:1 acetonitrile: isopropanol; 2 - $37 \mathrm{~min}$, linear gradient to $30 \%$ water/ $70 \% 2: 1$ acetonitrile:isopropanol; $37-42 \mathrm{~min}$, linear gradient to 2:1 acetonitrile: isopropanol; $42-47 \mathrm{~min}$, linear gradient to $65 \%$ water/ $35 \% 2: 1$ acetonitrile: isopropanol. The flow rate of the column was $1.5 \mathrm{ml} \mathrm{min}^{-1}$, and the absorption of the protein coming off the column was monitored every $3 \mathrm{~s}$ using a Waters 991 photodiode array with $2 \mathrm{~nm}$ resolution.

The polypeptides came off the analytical column in a wellresolved fashion with the elution data from the wild-type preparation shown in Figure 2-3. The wild-type preparation contained five chromoproteins and 2 colorless polypeptides. The order of protein elution was $\mathrm{L}_{\mathrm{cm}}$ at $28 \mathrm{~min}, \beta^{\mathrm{AP}}$ at $28.5 \mathrm{~min}, \alpha^{\mathrm{PC}}$ along with a small amount of colorless polypeptide of greater than $30 \mathrm{kD}$ at $29 \mathrm{~min}, \beta^{\mathrm{PC}}$ at $30.5 \mathrm{~min}, \alpha^{\mathrm{AP}}$ at $33 \mathrm{~min}$, and $\beta^{18}$ at $36 \mathrm{~min}$. The second colorless polypeptide of approximately $30 \mathrm{kD}$ eluted at about $31.5 \mathrm{~min}$.

\section{SDS-PAGE}

One dimensional polyacrylamide gel electrophoresis was carried out on both intact PBS, $18 \mathrm{~S}$ core preparations, and HPLC fractions. The method was based on that of Laemmli (Laemmli, 1970) and 


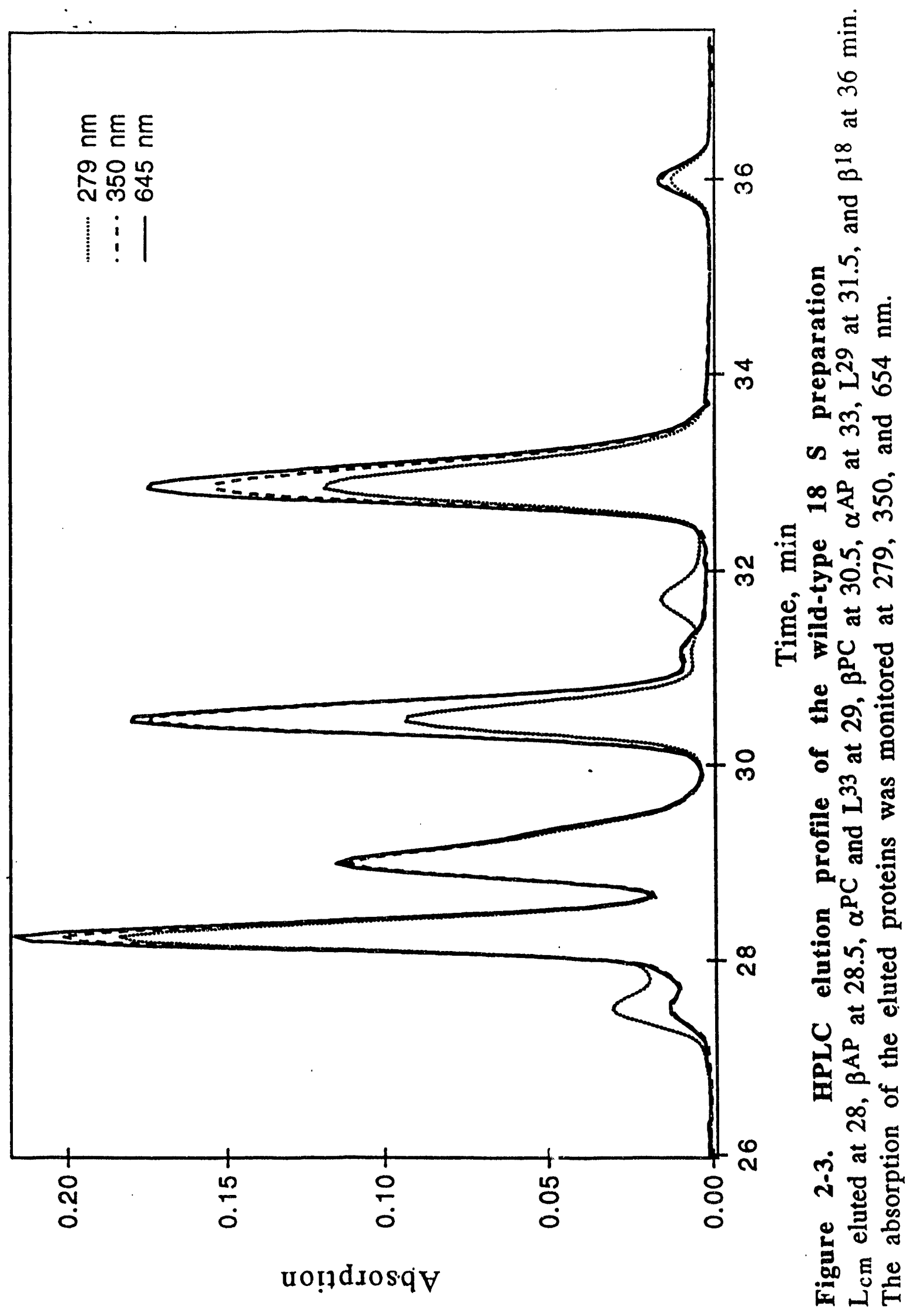


was carried out as outlined in Maxson's thesis. The equipment used was from Hoeffer Scientific Instruments (San Francisco, CA). The stock solutions used are given in Table 2-3 with the recipe used for a $15 \%$ running gel with a $5 \%$ stacking gel given in Table 2-4.

The gels were cast using two $16 \times 18 \mathrm{~mm}$ glass plates separated by spacers of the appropriate width and held upright in a casting stand. The glass plates were carefully cleaned using water and ethanol to eliminate any fingerprints, and everything was handled using latex gloves.

The solutions for the running and stacking gels were made up just prior to pouring the gel, with the initiators added last. When pouring both gels special care was taken to eliminate any air bubbles. Special care needs to be taken with the acrylamide since it is a neurotoxin.

The running gel was allowed to polymerize for at least $90 \mathrm{~min}$ before adding the stacking gel. In the interim, a small amount of water was carefully layered on the top of the running gel to ensure an even, bubble-free surface at the interface. The water was removed before pouring the stacking gel. The stacking gel was poured, and a mold for the sample wells was carefully inserted in the gel to create the sample lanes. Once the stacking gel polyr.erized (after $30 \mathrm{~min}$ ), the mold was carefully removed and the wells were rinsed with tank buffer.

Protein samples (containing approximately $40 \mu \mathrm{g}$ of protein) were prepared by mixing equal volumes of protein solution and $20 \%(w / v)$ trichloroacetic acid. The mixture was kept at $4{ }^{\circ} \mathrm{C}$ for a minimum of one hour and then centrifuged to recover the precip- 
Table 2-3

Solutions Needed For SDS-PAGE

Monomer Solution (Stored at $4^{\circ} \mathrm{C}$ in Dark)

Acrylamide

Bis-Acrylamide

$\mathrm{H}_{2} \mathrm{O}$

Tris

$\mathrm{H}_{2} \mathrm{O}$

Tris

$\mathrm{H}_{2} \mathrm{O}$

SDS

$\mathrm{H}_{2} \mathrm{O}$

Initiator

(Made Fresh with each gel)
Ammonium persulfate $\mathrm{H}_{2} \mathrm{O}$
$58.4 \mathrm{~g}$

$1.6 \mathrm{~g}$

to $200 \mathrm{ml}$

$36.3 \mathrm{~g}$

to $200 \mathrm{ml}$

$3.0 \mathrm{~g}$

to $50 \mathrm{ml}$

$50 \mathrm{~g}$

to $500 \mathrm{ml}$

2X Treatment Buffer

( Stored at $-20^{\circ} \mathrm{C}$ ) Tris

$2.5 \mathrm{ml}$ of Stacking Gel

Buffer

SDS

$4.0 \mathrm{ml}$ of $10 \%$ SDS

Glycerol

Solution

2-mercaptoethanol

$2.0 \mathrm{ml}$

$1 \%$ Bromophenol Blue $20 \mu 1$

Solution

$\mathrm{H}_{2} \mathrm{O}$

to $10 \mathrm{ml}$ 
Table 2-3 (cont)

Tank Buffer

(Made Fresh with each gel)

Tris

Glycine

SDS

$12.0 \mathrm{~g}$

$57.6 \mathrm{~g}$

$40 \mathrm{ml}$ of $10 \%$ SDS

Solution

$\mathrm{H}_{2} \mathrm{O}$ to 4.01

Stain Stock

Coomassie Blue R-250 $2.0 \mathrm{~g}$

$\mathrm{H}_{2} \mathrm{O}$

to $200 \mathrm{ml}$

Stain

Coomassie Blue R-250 $62.5 \mathrm{ml}$ of Stain Stock

Methanol

$250 \mathrm{ml}$

Acetic Acid

$50 \mathrm{ml}$

$\mathrm{H}_{2} \mathrm{O}$

to $500 \mathrm{ml}$

Destaining Solution I

Methanol

Acetic Acid

$\mathrm{H}_{2} \mathrm{O}$

Destaining Solution II

Acetic Acid

Methanol

$\mathrm{H}_{2} \mathrm{O}$

$140 \mathrm{ml}$

\section{Molecular Weight}

Standards

(Heated for $5 \mathrm{~min}$ at $100^{\circ} \mathrm{C}$, stored at $-20^{\circ} \mathrm{C}$ )

Bio-Rad Standard

Protein Mixture

Buffer

$100 \mu \mathrm{l}$ of $2 \mathrm{X}$

Treatment Buffer

$\mathrm{H}_{2} \mathrm{O}$
$100 \mathrm{ml}$

to 2.01

$10 \mu 1$

$100 \mathrm{ml}$

to 1.01

$90 \mu 1$ 
Table $2-4$

Recipe for 15\% Running Gel/ 5\% Stacking Gel

\begin{tabular}{|c|c|c|}
\hline Stock Solution ${ }^{2}$ & $15 \%$ Running Gel & $5 \%$ Stacking Gel \\
\hline Monomer Solution & $7.5 \mathrm{ml}$ & $1.0 \mathrm{ml}$ \\
\hline Running Gel Buffer & $4.0 \mathrm{ml}$ & \\
\hline Stacking Gel Buffer & & $1.5 \mathrm{ml}$ \\
\hline $\mathrm{H}_{2} \mathrm{O}$ & $3.3 \mathrm{ml}$ & $3.4 \mathrm{ml}$ \\
\hline $10 \%$ SDS Solution & $0.15 \mathrm{ml}$ & $0.06 \mathrm{ml}$ \\
\hline Initiator & $75 \mu l$ & $60 \mu l$ \\
\hline TEMED & $5 \mu \mathrm{l}$ & $3 \mu 1$ \\
\hline
\end{tabular}

a Composition of stock solutions given in Table 2-3 
itate. The precipitate was resuspended in $20 \mu \mathrm{l}$ of $2 \mathrm{X}$ treatment buffer (recipe given in Table 2-3) and loaded into the sample wells using a $200 \mu \mathrm{l}$ pipetman. Empty sample wells were loaded with 20 $\mu l$ of $2 X$ treatment buffer. After loading the wells, tank buffer was carefully layered on top to exclude air.

The gel was run at a constant current of $20 \mathrm{~mA}$ until the tracking dye ran off the bottom. The gel was then removed from the casting glass plates and stained with the Coomassie Blue Stain overnight. The gel was destained in two steps; first, a rapid destaining with Destain solution I for 1- $2 \mathrm{~h}$, and then, slow destaining with Destain solution II overnight. After destaining the gel was stored in deionized water until it could be dried under vacuum.

\section{Spectroscopy Sample Preparation}

For absorption spectroscopy, samples of PBS and $18 \mathrm{~S}$ core preparations were taken directly off the sucrose gradients. The preparations were generally diluted to less than $1 \mathrm{~cm}^{-1}$ at the absorption maximum using Buffer A containing $1.0 \mathrm{M}$ sucrose for the PBS preparations, and Buffer C containing 0.9 M sucrose for the $18 \mathrm{~S}$ preparations. I have found that both preparations are generally more stable if they are diluted using buffer containing a high concentration of sucrose.

Whole cell samples were prepared by centrifuging cells near the end of the $\log$ phase of growth for $5 \mathrm{~min}$ at $10,000 \mathrm{rpm}$ in a Dupont SS-34 rotor at room temperature. The cells were immediately 
resuspended in fresh growth medium or $0.75 \mathrm{M}$ phosphate buffer pH 7.0 such that the absorption of the samples was less than $2 \mathrm{~cm}^{-1}$ at $630 \mathrm{~nm}$.

Steady state fluorescence samples of PBS and $18 \mathrm{~S}$ core preparations for room temperature work were diluted to an absorption of less than $0.12 \mathrm{~cm}^{-1}$ at the excitation wavelength using the appropriate buffer in $1.0 \mathrm{M}$ sucrose. Samples of whole cells were diluted to less than $0.15 \mathrm{~cm}^{-1}$ at $590 \mathrm{~nm}$ using fresh growth medium or $0.75 \mathrm{M}$ phosphate buffer, $\mathrm{pH} 7$.

Time-resolved fluorescence spectroscopy samples were prepared as for steady state fluorescence. Fresh samples of PBS and $18 \mathrm{~S}$ core preparations were prepared approximately every hour to minimize sample degradation with special care taken to keep the same concentration of protein.

Samples for low temperature spectroscopy were prepared by diluting a minimum volume of sample in $2.0 \mathrm{M}$ sucrose $(\mathrm{H}$. Scheer, personal communication). The sample was put under house vacuum for approximately $10 \mathrm{~min}$ prior to freezing to remove most of the gases present. Then the sample was frozen in a $1 \mathrm{~cm}$ disposable plastic cuvette without any precooling by simply dropping the cuvette into a square optical dewar containing liquid nitrogen. The sucrose concentration is critical; the best glasses are obtained with the highest concentration of sucrose. For some of the $18 \mathrm{~S}$ preparations, I actually concentrated the protein before freezing in the sucrose solution. Other cryoprotectants were tried including PEG-4000 (Mimuro, Yamazaki et al., 1989) which gave a 
snow and 50\% glycerol which caused immediate uncoupling of proteins in intact PBS as judged by fluorescence spectroscopy.

\section{Steady-State Spectroscopy}

Absorption spectroscopy was carried out on an AVIV 14 DS spectrophotometer with uv-vis-nir capability (AVIV, Lakewood, NJ) or on a Varian 2300 spectrophotometer (Varian, Houston, TX) interfaced with a Macintosh SE using Labview 2 (National Instruments Corp., Austin, TX). Slight modifications were made on the AVIV instrument to obtain intact cell absorption spectra with minimal scattering problems (Myers, Graham et al.,1980). Both reference and sample PMTs were covced with a single layer of translucent paj $\mathrm{r}$, and the cuvette holders were placed as close to the PMT as possible. The spectra obtained were corrected by subtracting the absorption present at $720 \mathrm{~nm}$.

Steady-state fluorescence spectra were taken on a Spex Fluorolog fluorimeter (Spex Industries, Edison, NJ) interfaced with an Epson computer. Excitation spectra and quantum yield measurements were corrected for lamp intensity using 1,1',3,3,3',3'-hexamethylindotricarbocyanine perchlorate (HITC, $8 \mathrm{~g} \mathrm{l}^{-1}$ in acetonitrile, Exciton, Dayton, $\mathrm{OH}$ ) as a quantum reference counter (Nothnagel, 1987) with a Hamamatsu R928 photomultiplier (Hamamatsu Industries, Japan). 
Time-Resolved Fluorescence Spectroscopy

Time-resolved fluorescence spectroscopy was carr $\downarrow$ out using the reverse-timing, single-photon counting system ( $F$; sre 2-4) described in Mukerji's thesis (Mukerji, 1990). It is ve\% similar to the standard single-photon-counting instrument found the literature. An excellent discussion of single-photon countin ${ }_{\xi}$ san also be found in a book by $\mathrm{O}^{\prime}$ Connor and Phillips ( $\mathrm{O}^{\prime}$ Connor and hillips, 1984). I will give a brief description of our instrument. in in the following paragraphs.

A Spectra-Physics (Mountain View, CA) model 2040 ion mode-locked laser pumps a dye laser (Spectra-Physics, del 375) at $82 \mathrm{MHz}$ and $0.5 \mathrm{~W}$ with a pulse duration of 100-120 Approximately $100 \mathrm{~mW}$ of the $\mathrm{Ar}$ ion beam is split off to a phc iode to provide a trigger puise for the cavity dumper driver be the beam enters the dye laser. The dye laser output is cav dumped (Spectra-Physics, Model 344) at a frequency of $4 \mathrm{MHz}$ wi 5 to 25 $\mathrm{mW}$ of power and a pulse duration of $6 \mathrm{ps}$. Part of the $\mathrm{d}$ beam is split off to provide a stop pulse for the time-to-amplitud converter $(t a c)$ while the rest enters the sample box and tes the sample fluorescence.

The sample fluorescence is collected at $90^{\circ}$ to the exciti 1 beam using a camera lens (Nikon) to focus the photons on the ac iv monochromator (SA Instruments, New Jersey) and then to Hamamatsu MCP-PMT, model R2809U-11 with channels 6 , in diameter. No more than 4000 counts per second are allowe in the MCP-PMT during data collection to prevent multiple photo: vents and to prolong the lifetime of the detector. The MCP-PMT ovides 
TAC Time-to-Amplitude Convertor MCA MultiChannel Analyzer

M Mirror

BS Beam Splitter

PD Photodiode

S Sample

L Lens
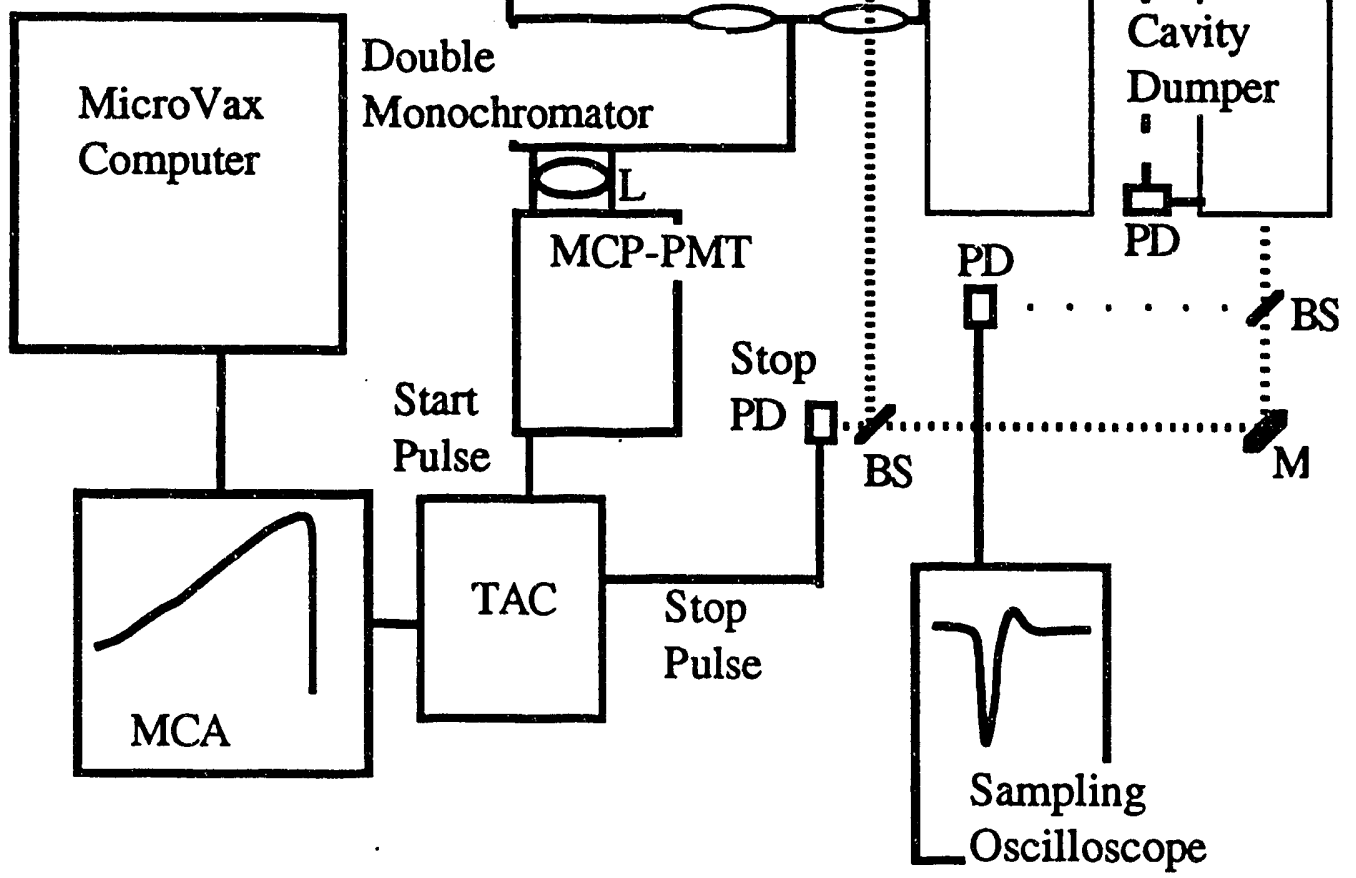

Figure 2-4. Instrumental Setup for Single Photon Timing Laser System. 
a pulse for each photon it 'sees'; the pulse is amplified with a Hewlett Packard amplifier, discriminated (Tennelec), and sent to the tac.

The tac starts a voltage ramp with the input of the MCP-PMT pulse and stops the ramp with the input of the stop pulse from the photodiode. The voltage which contains the information about the length of time between the start and stop photons is then sent to a multichannel analyzer (Northern Tracor, Madison, WI), and a count is added to the bin or channel corresponding to that voltage. This entire process is repeated thousands of times during the course of the experiment until there is a maximum of 10,000 counts in the peak channel. It can take from $5 \mathrm{~min}$ to $2 \mathrm{~h}$ to collect a decay depending upon the fluorescence yield of the sample, the length of the lifetimes involved, and the width of the channels on the multichannel analyzer.

The histogram that is built up using the 1024 channels of the multichannel analyzer is transferred to the VAX for data analysis. An instrument response function (irf) is collected just prior to or immediately after each decay measurement using dilute non-dairy creamer as a scattering solution. The width of the irf varies from 45 ps to 70 ps depending upon aperture of the camera lens and monochromator slit widths. The narrower irfs show more structure and are harder to deconvolute for a good fit. The time resolution of the instrument is generally considered to be one-fifth the width of the irf.

Fine adjustments are made to the dye laser and cavity dumper using a fast-triggering oscilloscope (Tektronic) to view the pulse. 
The time window of the tac can be adjusted from a minimum of 5 ps per channel to $\mu$ s per channel. For my experiments it is set at 10 to $15 \mathrm{ps}$ per channel giving a total time window of 10 to $15 \mathrm{~ns}$. The excitation wavelength can range from $580 \mathrm{~nm}$ to $640 \mathrm{~nm}$ with Rhodamine 590 dye or $610 \mathrm{~nm}$ to $715 \mathrm{~nm}$ with DCM dye (Exciton, $\mathrm{OH})$.

\section{Data Analysis of Time-Resolved Fluorescence Decays}

The kinetics of phycobiliprotein excited states is expected to be fit by a sum of exponentials according to modeling done by Holzwarth (Holzwarth, Wendler et al., 1987). The fluorescence decays were fit to a sum of exponentials using a software program kindly provided by A. Holzwarth. The program uses a semilinear Marquardt algorithm (Marquardt, 1963). The program calculates the sum of exponentials and convolutes that function with an instrument response function, and the results of the convolution are compared to the experimental decay and a reduced $\chi^{2}$ is calculated. The program repeats the iterative reconvolution procedure until the $\chi^{2}$ value is at a minimum. The instrument response function, or irf, used in the data analysis is collected just prior to or immeditately after the experimental decay using a scattering solution of nondairy creamer.

Figure 2-5 shows an example of a typical decay with its accompanying irf; the arrows indicate the start and end of the data fit. The data were fit to two, three, or four exponentials as shown in Figure 2-6. The goodness of a fit is judged not only by the $\chi^{2}$ value 


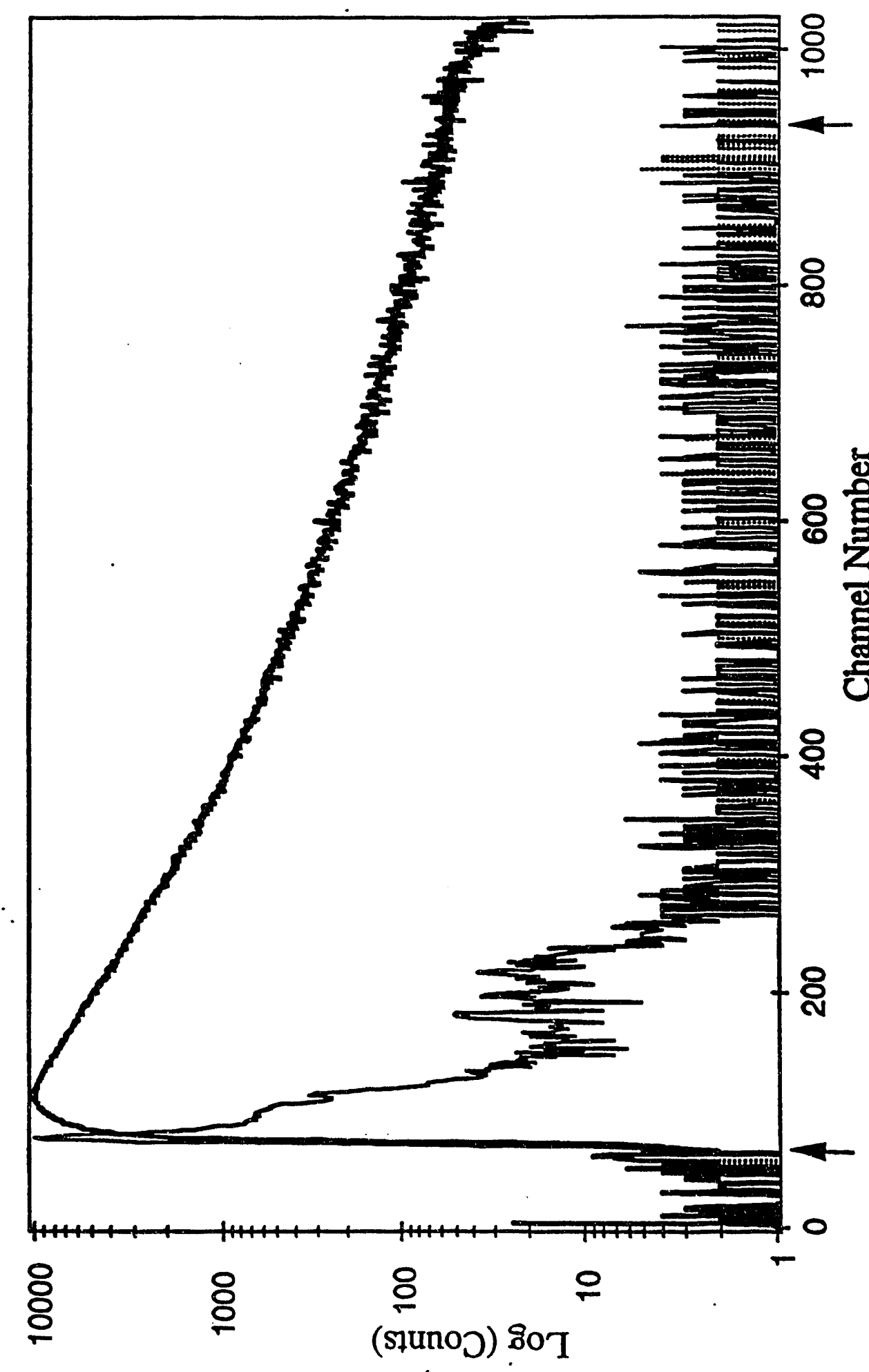

过

$\dot{U} \equiv$

d an

约

王宅

.

运是

๙ิ

उ롱

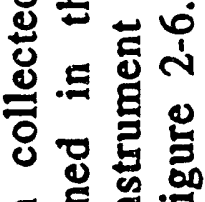

도…

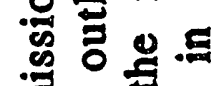

웡

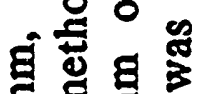

ฮ

กิ้

额慁是

త్

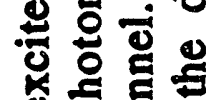

ชิ

등

舟

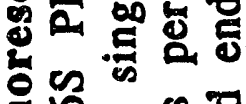

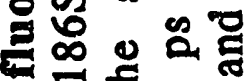

-

ส

ô

응용요

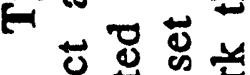

过 过 ஸ. ง ○远的总

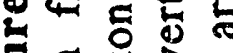

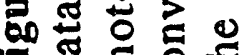
国䓃苍 
which should be less than 1.2 but also by the appearance of the residuals. The residuals should not have any systematic deviations present as shown in the two component fit and, to a lesser extent, the three component fit in Figure 2-6. The appearance of the early part of the residual is especially critical; if the sum of exponentials used to fit the data is missing a very fast component the early part of the residual plot will show the fit deviating wildly from the experimental data.

Other factors are also important for a good fit. First, the laser system should be very stable before collecting data such that the pulse shape doesn't vary between the irf and the decay. Also, the bias level on the tac has a strong influence on the fit; we have found that the bias level on our tac must be set within a narrow range otherwise the tac displays nonlinear behavior, and the resulting decays give residuals with almost sinewave-like features.

A third factor to consider is the width of the irf; when the irf is greater than 60 ps the program is able to fit the experimental data with lower $\chi^{2}$ values. When the apertures and slits are closed to give narrower irfs the fits worsen. This effect could be due to two different factors; either the wider irf tends to mask any small laser instabilities, or the fitting program just isn't as efficient at fitting the prominent features present in the narrower irfs. Also, it's possible that the laser is more unstable when the pulse width is made as small as possible. All of these factors need to be considered prior to data collection when designing the experiment.

The data fits shown in Figure 2-6 show some of the problems of interpreting the fit. The two-component fit is obviously not a good 
2 Components

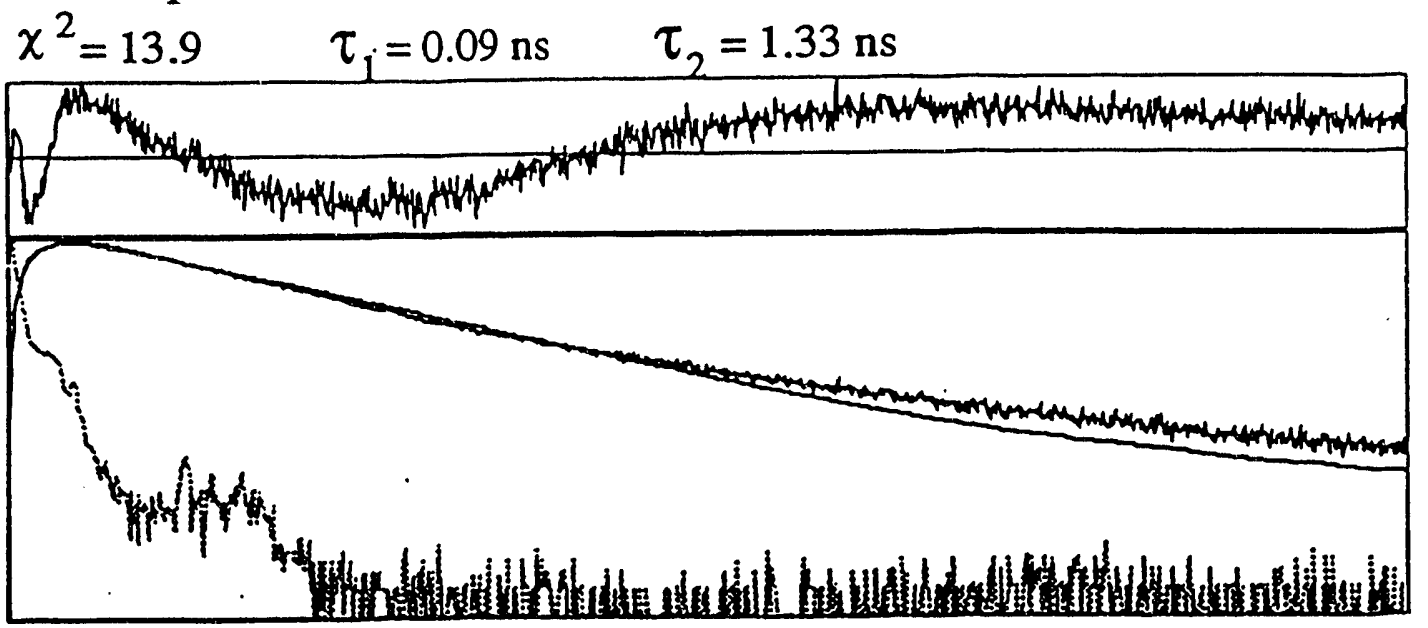

\section{Components}

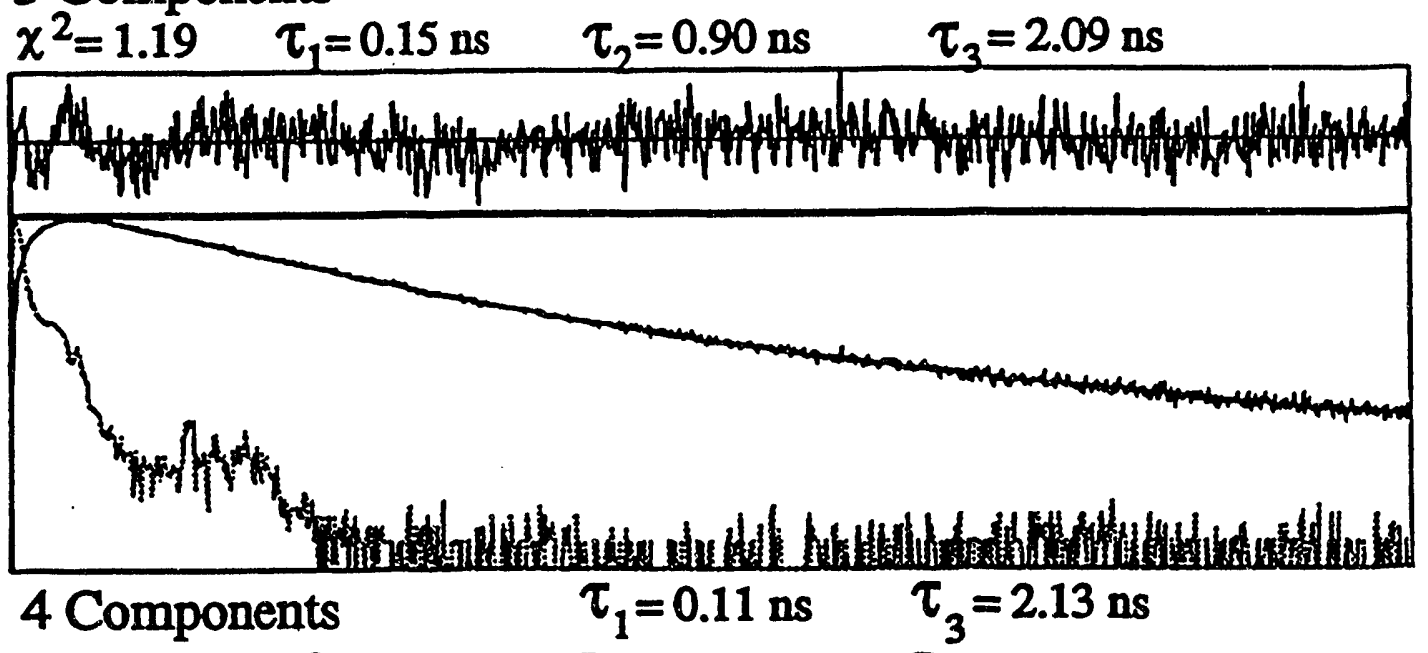

$$
\chi^{2}=1.16 \quad \tau_{2}=0.93 \mathrm{~ns} \quad \tau_{4}=0.07 \mathrm{~ns}
$$

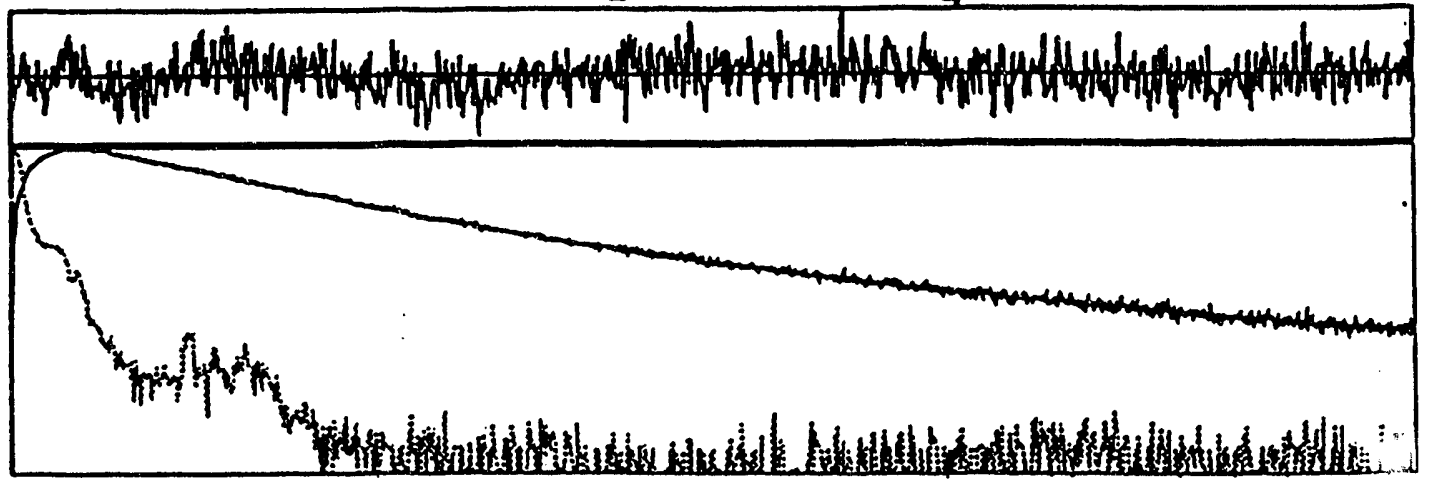

Figure 2-6. Typical exponential fits to data

2,3 , or 4 exponential fits to data displayed in Figure 2-5. 
fit to the decay, as shown by both the $\chi^{2}$ value and the residual. The three-component fit is indistinguishable from the four component fit in terms of the $\chi^{2}$ value, but the residual isn't quite as good for the three-component fit. At times, it becomes very difficult to differentiate between three and four lifetimes; I always go with the minimum number of components if there is no obvious improvement with the addition of one more lifetime.

For more meaningful data analysis, global analysis is generally carried out in which all of the decays collected for the sample are fit simultaneously. Typically, sets of decays are taken with one excitation wavelength and emission wavelengths spanning the emission spectrum of the sample. Each decay is first fit individually using the iterative deconvolution software to obtain approximate lifetimes. Then the decays are analyzed as a set using a global optimization algorithm provided by $\mathrm{A}$. Holzwarth. The algorithm assumes that the fluorescent lifetimes are independent of emission wavelength, with the amplitude of the lifetime varying with wavelength (Holzwarth, Wendler et al., 1987; Knorr and Harris, 1981; Knutson, Beechem et al., 1983). This reduces the number of parameters allowed to vary during the fit, so the fits should be more meaningful. The global software is also better at finding the very short lifetimes present, especially when the lifetime has a small or zero amplitude at many wavelengths. Global analysis also works better for finding risetimes or negative amplitudes.

Risetimes are present when energy is transferred from one pigment pool to another pool at a lower excited state energy; the 
fluorescence of the second pool is delayed by a time consistent with the lifetime of the first pigment pool. I will present all the global analysis work in the form of decay-associated-spectra or DAS. DAS show the amplitude or yield of the individual components at each wavelength examined. 


\section{References for Chapter 2}

Bryant, D. A., R. de Lorimier, G. Guglielmi, and S. E. Stevens, Jr. (1990). Arch. Microbiol. 153: 550-560.

Gingrich, J. C., D. J. Lundell and A. N. Glazer. (1983). J. Cell. Biochem. 22: $1-14$.

Holzwarth, A. R., J. Wendler and G. W. Suter. (1987). Biophys. J. 51: $1-12$.

Knorr, F. J. and J. M. Harris (1981). Anal. Chem. 53: 272-276.

Knutson, J. R., J. W. Beechem and L. Brand. (1983). Chem. Phys. Lett. 102: 501-507.

Laemmli, U. K. (1970). Nature 227: 680-685.

Marquardt, J. (1963). J. Soc. Ind. Appl. Math 11: 431-441.

Maxson, P. (1988). Ph. D. Thesis, University of California, Berkeley. Lawrence Berkeley Laboratory Report, LBL-26163.

Mimuro, M., I. Yamazaki, N. Tamai and T. Katoh. (1989). Biochim. Biophys. Acta 973: 153-162.

Mukerji, I. (1990). Ph. D. Thesis, University of California, Berkeley. Lawrence Berkeley Laboratory Report, LBL-30136.

Myers, J., J. R. Graham and R. T. Wang. (1980). Plant Physiol. 66: 1144-1149.

Nothnagel, E. A. (1987). Anal. Biochem. 163: 224-237.

O'Connor, D. V. and D. Phillips (1984). Time-Correlated Single Photon Counting. London, Academic Press.

Swanson, R. V. and A. N. Glazer (1990). Anal. Biochem. 188: 295299. 
Yamanaka, G., D. J. Lundell and A. N. Glazer. (1982). J. Biol. Chem. 257: $4077-86$.

Zhou, J. (1991). Ph. D. Thesis, Pennsylvania State University. 
Chapter 3. Discussion of the Whole-Cell Results

I will start by first examining the steady state and timeresolved spectroscopy of healthy intact cells from the different strains. In this chapter I will compare the efficiency of energy transfer to the reaction centers in the wild-type and mutant cells. I will try to address the following questions in this chapter. Does the amount of phycobiliprotein present in the cells relative to $\mathrm{chl} a$ change because of the mutation? Do the antennae from the mutants 'leak' more energy than the wild-type PBS? Are the energy transfer kinetics from the PBS to the reaction centers altered with the mutation?

\section{Absorption Spectroscopy}

Absorption spectroscopy was carried out to quantify the amount of phycobiliprotein present in the cells relative to chl a. I cannot distinguish between free phycobiliprotein and intact PBS absorption in the cells.

The spectrophotometer's measuring beam is easily scattered from the intact cells, and modifications to the sample box were necessary to obtain relatively scatter-free absorption spectra. The first change made was to cover the windows between the sample and reference holders and the PMT with a translucent paper. The translucent paper causes the measuring light to be more diffuse on the PMT; this makes the position of the measuring beam on the detector less critical to the measurement. In a paper by Myers, Graham, and Wang, they use an opaque Plexiglas in this manner, 
but I found a single layer of translucent paper (weighing paper in this case) to work well (Myers, Graham et al., 1980). Alternatively, opal glass can be placed between the sample and the detector, but the corrected background turned out to be more linear with the translucent paper. The opal glass probably scattered the light too much.

The second modification to the spectrophotometer's sample box was to move the sample holders as close as possible to the PMT to minimize the loss of light due to scattering. I also corrected for background scatter by subtracting the absorption at $720 \mathrm{~nm}$, a portion of the spectrum where there should be little absorption (Myers, Graham et al., 1980). Without the changes to the sample box mentioned above the absorption spectra had huge backgrounds due to light scatter with an $1 / \lambda^{2}$ function.

Corrected absorption spectra for whole cells are shown in Figure 3-1a,1b. The cultures were one week old, and all appeared to be healthy and in the $\log$ phase of growth. The cultures were grown simultaneously under the same light and with the same $\mathrm{CO}_{2}$ and $\mathrm{N}_{2}$ mixture. All of the absorption spectra contained two peaks; peak I at $631-633 \mathrm{~nm}$ and peak II at $677-680 \mathrm{~nm}$. The absorption maxima from Figures 3-1a,1b are given in Table 3-1.

As mentioned previously, one of the goals of these experiments was to calculate the relative amount of phycobiliprotein to chl $a$. I was interested in seeing if some of the mutations would cause cells to produce relatively more chl $a$ to compensate for inefficient PBS energy transfer. The amount of phycobiliprotein present in the cells is shown by the $630 \mathrm{~nm}$ peak while the chl $a$ concentration is 


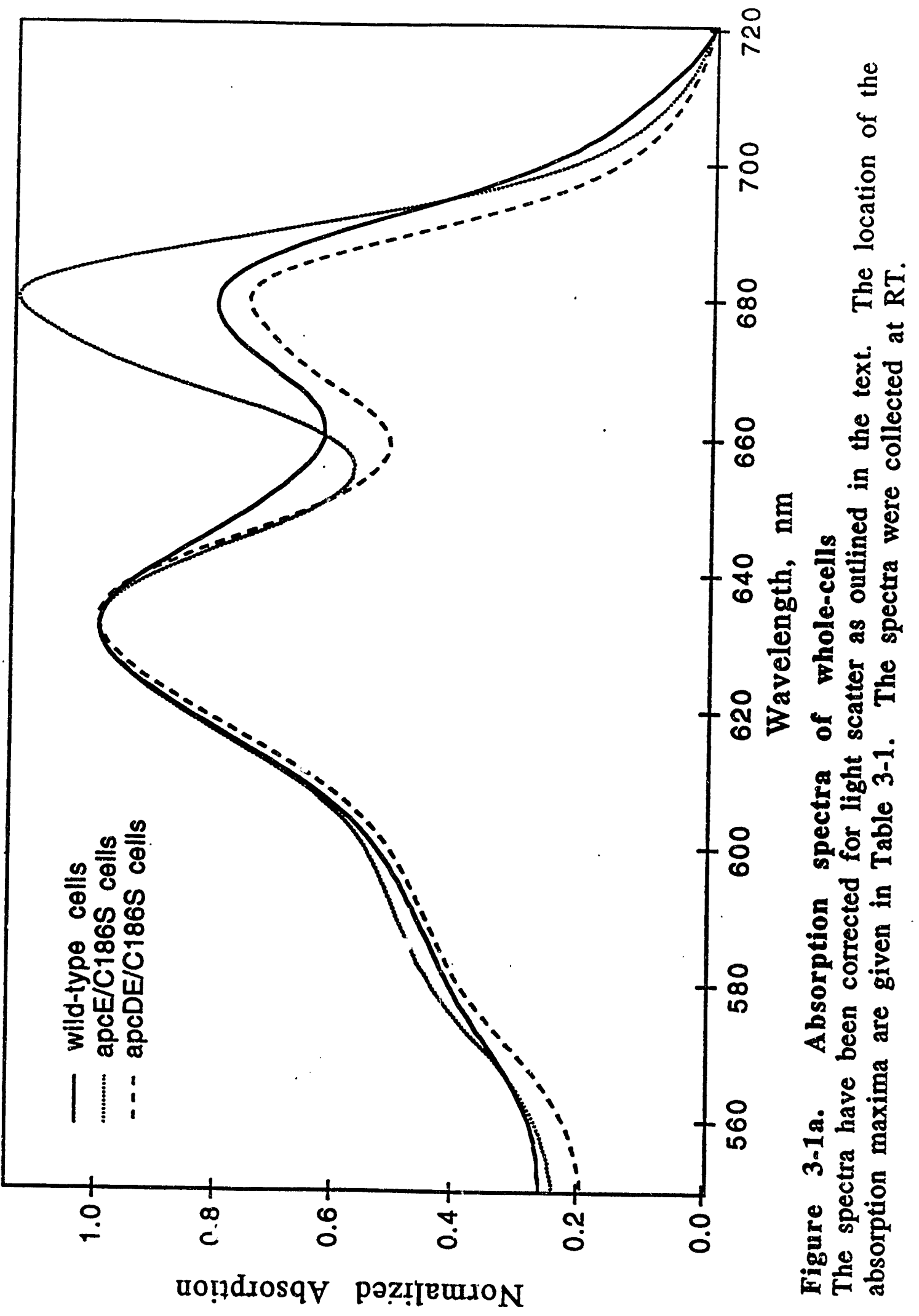




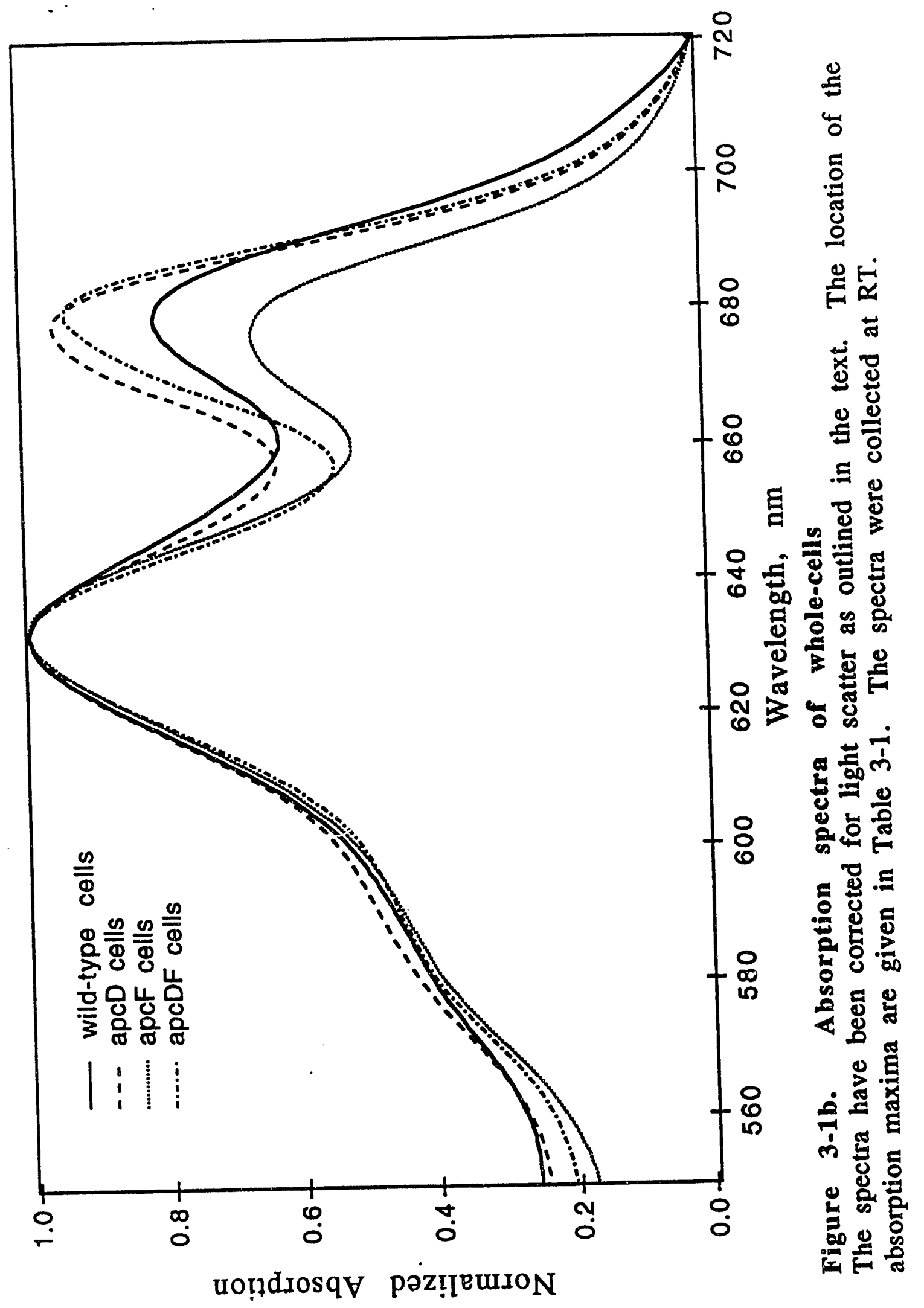


Table 3-1

Spectral Data for the Intact Cells

\begin{tabular}{cccc} 
& Absorption Maxima & Emission Maxima \\
\cline { 2 - 3 } wild-type cells & $632 \mathrm{~nm}$ & $657 \mathrm{~nm}$ \\
apcD cells & $679 \mathrm{~nm}$ & & \\
& $632 \mathrm{~nm}$ & $659 \mathrm{~nm}$ \\
apcF cells & $678 \mathrm{~nm}$ & & \\
& $633 \mathrm{~nm}$ & $659 \mathrm{~nm}$ \\
apcDF cells & $677 \mathrm{~nm}$ & & \\
& $632 \mathrm{~nm}$ & & $651 \mathrm{~nm}$ \\
apcE/C186S cells & $680 \mathrm{~nm}$ & & \\
& $632 \mathrm{~nm}$ & $650 \mathrm{~nm}$ \\
apcDE/C186S cells & $680 \mathrm{~nm}$ & $705 \mathrm{~nm}$ \\
& $633 \mathrm{~nm}$ & $653 \mathrm{~nm}$
\end{tabular}


shown by the $680 \mathrm{~nm}$ peak in the absorption spectrum. As shown in Figures 3-1a,1b, there isn't any apparent trend in the relative amounts of each type of antenna complex present.

I found that the ratios of the two peaks fluctuated with experiment in all of the strains including that of the wild-type. I attribute this fluctuation to changing culture conditions, with the number and composition of the PBS to depend upon the culture age. For example, if the culture has been growing for several days and the cells are starting to get relatively thick, the amount of light available per cell will decrease due to shading. The Synechococcus sp. PCC cyanobacterium possesses a mechanism for adjusting the rod length to compensate for the variable light conditions (de Lorimier, Smith et al., 1992). At low light intensities the rod length increases, which results in a higher 630 to $680 \mathrm{~nm}$ absorption ratio. Also, work done by various laboratories show that the phycobiliproteins can be used as carbon and nitrogen sources during periods of cell starvation (Bryant, 1991; Stevens, Balkwill et al., 1981).

It is hard to correct for the differences in phycobiliprotein concentrations due to cell shading and nutrient availability, because some of the mutants have much different doubling times from that of the wild-type strain, see Table 2-2. Therefore, even though the different strains are grown under the same lights and in the same medium, the composition of the antenna can be quite different depending upon the stage of growth of the culture. I will keep this limitation in mind when discussing the spectral data for the intact cells in this chapter. 
Steady State Emission

With steady state emission spectroscopy one should be able to judge the efficiency of energy transfer from the PBS antenna to the PSII reaction center in whole-cells. If energy transfer to the reaction center is not occurring, the fluorescence yield of the PBS in the cells should increase; photochemistry is no longer successfully competing with fluorescence. There are some difficulties in interpreting the data. If the amount of uncoupled phycobiliprotein fluctuates during the growth of the culture, the fluorescence quantum yield of the cell also fluctuates. I define uncoupled phycobiliprotein to be either intact PBS uncoupled from reaction centers or dissociated hexamers or trimers of phycobiliprotein. Intact PBS uncoupled from reaction centers fluoresces from 650 to $680 \mathrm{~nm}$, depending upon the strain as shown in Chapter 4. Dissociated PC hexamers fluoresce around $650 \mathrm{~nm}$, while AP trimers emit at $660 \mathrm{~nm}$.

Relative quantum yields and corrected emission spectra were obtained for wild-type and mutant cells. The cells were illuminated at $580 \mathrm{~nm}$ to excite the PC in the rods while directly exciting only a small amount of the chl $a$ present.

As with the absorption spectra, the appearance of the emission spectrum and the quantum yield of all of the strains varied with culture age. The $\mathrm{apcD}$, $\mathrm{apcF}$, and apcDF fluorescence yields were comparable to that of the wild-type cells while the apcDE/C186S and apcE/C186S yields were roughly $150 \%$ of wild-type. There does appear to be some fluctuation in the amount of uncoupled PBS or dissociated phycobiliprotein present in the cells. The quantum 
yields of the mutants relative to that of the wild-type cells differed considerably with experiment. As an aside, the absolute fluorescence yields of the cells are considerably less than those of the isolated PBS in the wild-type, apcD, apcF, and apcDF strains. Most of the energy collected by the PBS in these strains goes to the reaction centers to carry out photochemistry.

The emission spectra are shown in Figures $3-2 a, 2 b$, and they are normalized at the emission maximum. The location of the emission maxima are given in Table 3-1. The emission spectra for the different strains were obtained under identical conditions with the same exciting light intensity. I treated the cells in exactly the same way during the experiment, so the relative number of closed reaction centers present in each strain should not differ if the process of energy transfer within the PBS is unchanged. To give the reader some idea of how much the emission spectrum can fluctuate with culture age and growing conditions, I have overlaid normalized wild-type emission spectra from several different experiments in Figure 3-3. The emis?ion spectra are from wild-type cultures between 4 and 8 days. There didn't seem to be any consistent trends with culture age. The intensity of the exciting light did vary somewhat with each experiment, since the spectra were taken at different times during the lifetime of the fluorimeter lamp.

Keeping these limitations in mind, I feel confident that the apcD, apcF, and apcDF spectra shown in Figure $3-2 b$ are indistinguishable from that of the wild-type while the apcDE/C186S and apcE/C186S spectra are different. The $\mathrm{apcD}$, apcF, apcDF, and wild-type emission spectra display two emission bands at approximately 650 


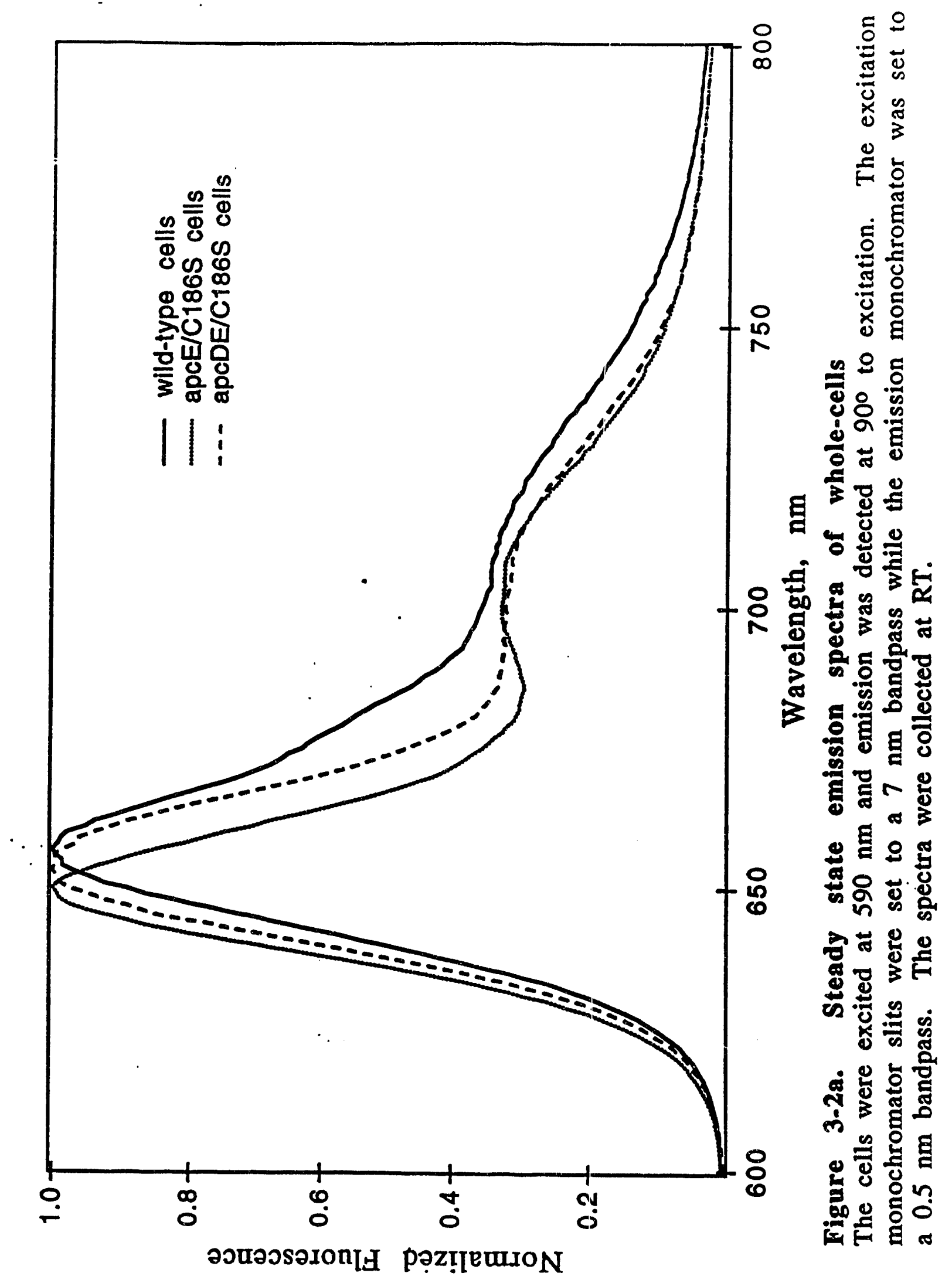




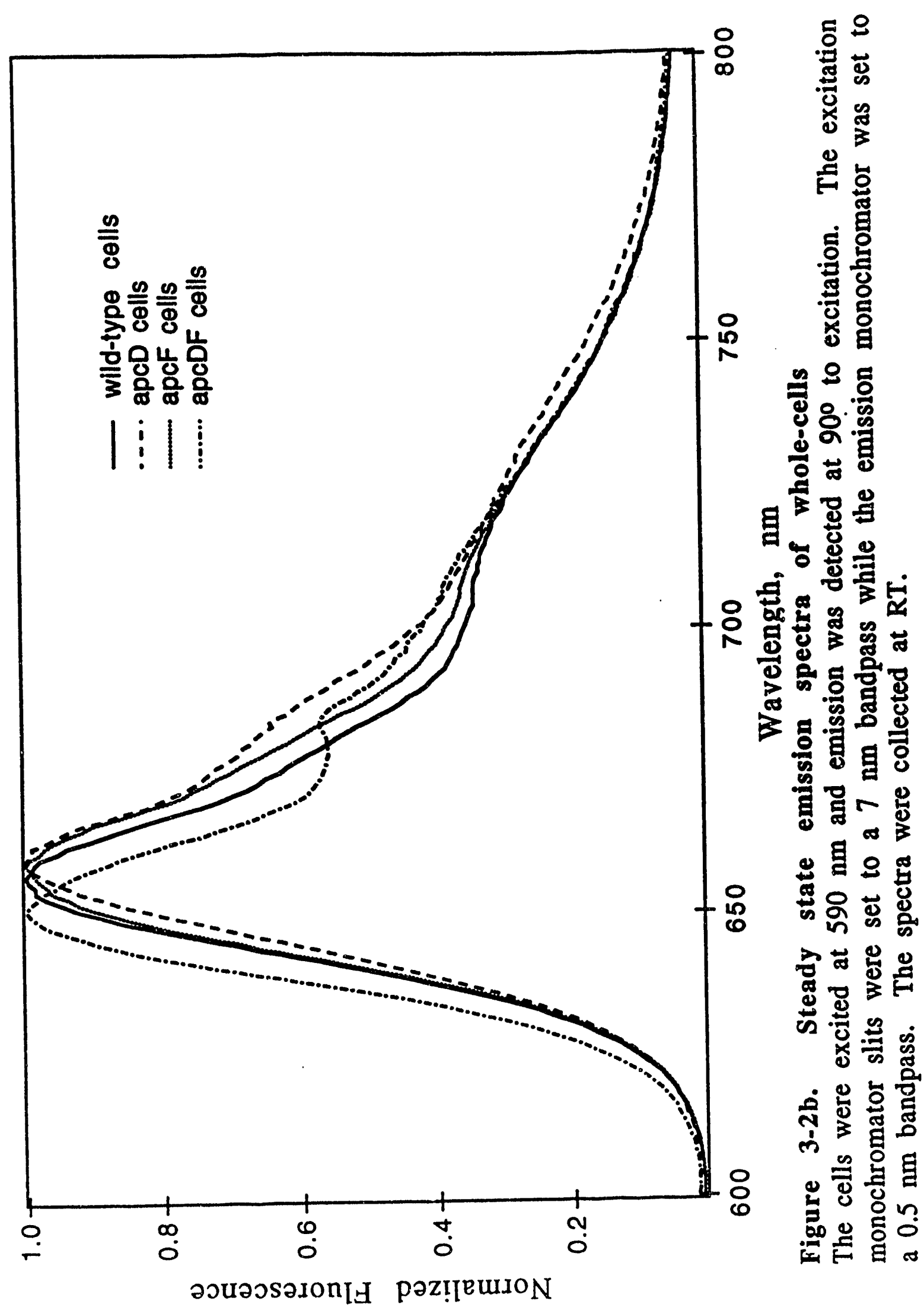




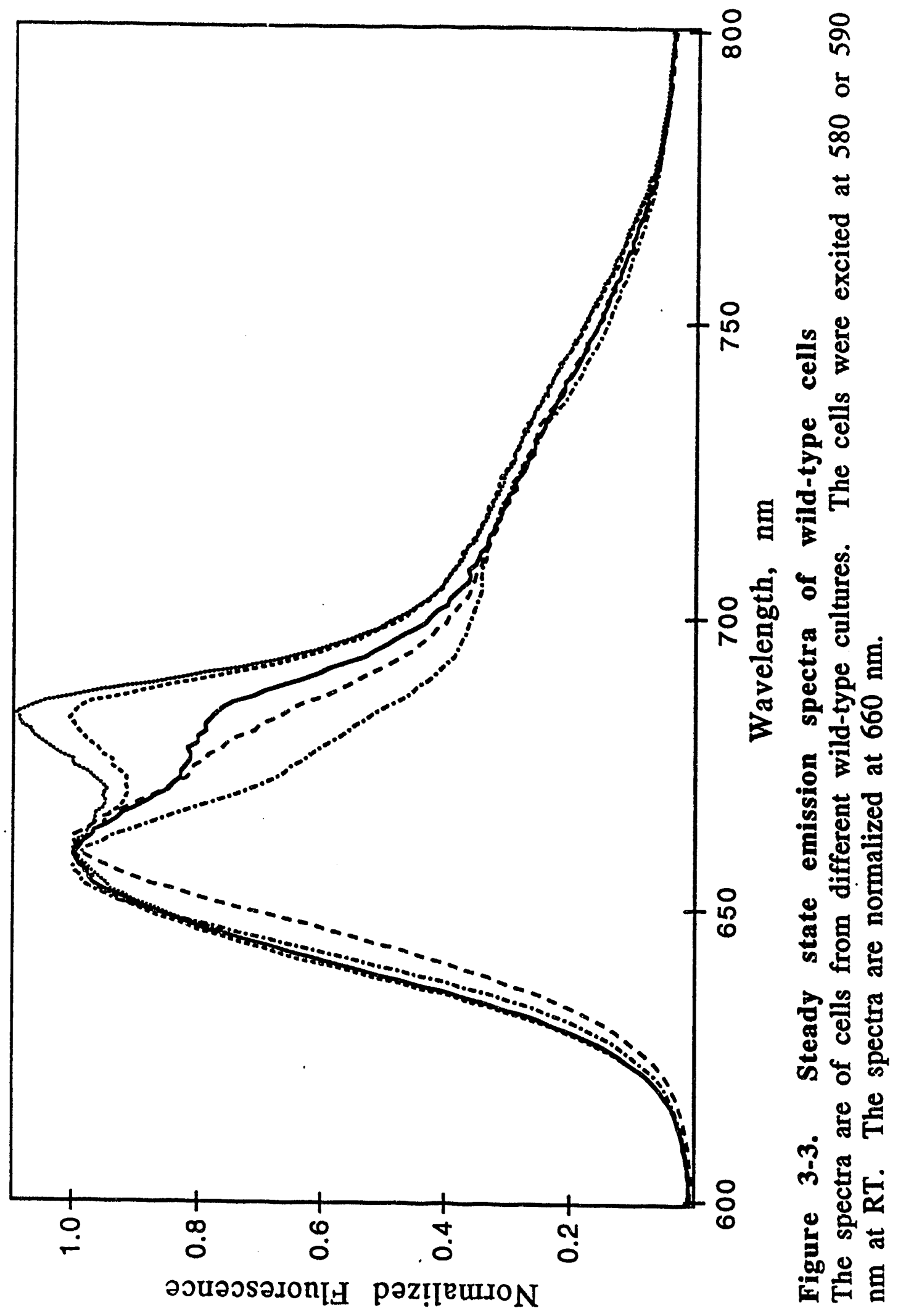


to $660 \mathrm{~nm}$ and $680 \mathrm{~nm}$. The first band at 650 to $660 \mathrm{~nm}$ is due mostly to the emission of dissociated phycobiliproteins with some contribution from intact PBS uncoupled from the reaction centers. Nothing else in the cell should fluoresce there. The amounts of dissociated phycobiliproteins present in the cells from the apcD, apcF, and apcDF mutants are roughly that of the wild-type as shown by the quantum yields. The second emission band at $680 \mathrm{~nm}$ must be due to a combination of chl $a$ fluorescence and emission from terminal emitters in uncoupled PBS.

In Figure 3-2a the emission spectra of the apcDE/C186S and apcE/C186S strains are displayed with the emission spectrum of the wild-type cells. The $680 \mathrm{~nm}$ emission shoulder in the wild-type spectrum is absent in the apcDE/C186S and apcE/C186S spectra. I have never observed any significant $680 \mathrm{~nm}$ emission in any of the whole-cells emission spectra I've taken with the apcDE/C186S and apcE/C186S mutants. Another difference between the apcE/C186S and wild-type emission spectrum is the presence of a $705 \mathrm{~nm}$ emitter in the apcE/C186S spectrum. This emission is due to the modified chromophore described in Chapter 1. The $705 \mathrm{~nm}$ emission band is not very prominent in the apcE/C186S spectrum, since it occurs at the same place in the spectrum as emission from some low lying excited states of PC (PC hexamers emit above 690 $\mathrm{nm}$ in addition to the main PC emission band at $650 \mathrm{~nm}$ ) along with chl $a$ emission. The presence of the modified chromophore is more prominent in the emission spectrum from the apcE/C186S PBS, as shown in the next chapter. Emission from the modified 
chromophore seems to be missing in the apcDE/C186S spectrum; it possibly is obscured by the PC and chl $a$ emission in the cells.

\section{Time-Resolved Fluorescence}

In a simpler world, one might be able to assign fluorescence lifetimes for the pigment pools present in the intact cells using time-resolved fluorescence. The fluorescence from a pigment pool will predominate at a certain place in the emission spectrum: 650 $\mathrm{nm}$ for PC, $660 \mathrm{~nm}$ for AP, and $680 \mathrm{~nm}$ for terminal emitters, PSII reaction centers, and chl $a$ The lifetimes of phycobiliproteins that transfer energy to reaction centers are much shorter than the emission decay lifetimes measured for the uncoupled phycobiliproteins. Energy transfer between coupled chromophores competes strongly with emission. Typical emission lifetimes for free phycobiliproteins are around $1.5 \mathrm{~ns}$; when the chromophores transfer energy to lower excited states their measured emission lifetimes decrease to less than $0.15 \mathrm{~ns}$. Energy transfer to a pigment pool can be seen by the appearance of a risetime or negative amplitude in the decay-associated spectrum, which indicates a delay in the appearance of emission from that pigment pool.

I will present the time-resolved fluorescence decay data as decay-associated spectra (DAS). The DAS are obtained from simultaneously analyzing the fluorescence decays from several different emission wavelengths using global analysis. With global analysis one obtains lifetimes and the relative yield of each lifetime 
component at different emission wavelengths. The lifetimes of the chromophores are assumed to be constant across the spectrum.

The risetime at $680 \mathrm{~nm}$ due to energy transfer to the reaction centers or terminal emitters is an important indicator of the efficiency of energy transfer. A longer risetime indicates less efficient transfer from outlying pigments to the terminal emitters because it is takes longer for the energy to reach the terminal emitters. I am making the assumption that small changes in the amount of PC present are not detected within the time resolution of the instrument.

The cells were excited at $580 \mathrm{~nm}$, and fluorescence decays were collected every $10 \mathrm{~nm}$ over the emission spectrum. The emission was collected for almost $12 \mathrm{~ns}$ after the exciting pulse. The DAS obtained from global analysis are presented in Figures 3-4a to 3-4f. A minimum of 5 lifetime components are necessary to fit the fluorescence decays for every strain.

The components resolved in the wild-type decays are very similar to those reported by other laboratories for dark-adapted cells of Synechococcus 6301 and Synechocystis sp. PCC 6803 (Mullineaux and Holzwarth, 1991; Bittersmann and Vermaas, 1991; Mullineaux, Bittersmann, et al., 1990). Based on their work, I assign the $0.050 \mathrm{~ns}$ component to AP and PC chromophore pools which are coupled by energy transfer to the terminal emitters. The $0.050 \mathrm{~ns}$ component emits in the blue region of the spectrum. The $0.16 \mathrm{~ns}$ component must be due to phycobiliproteins which transfer energy to the reaction center based upon this component's resolved 


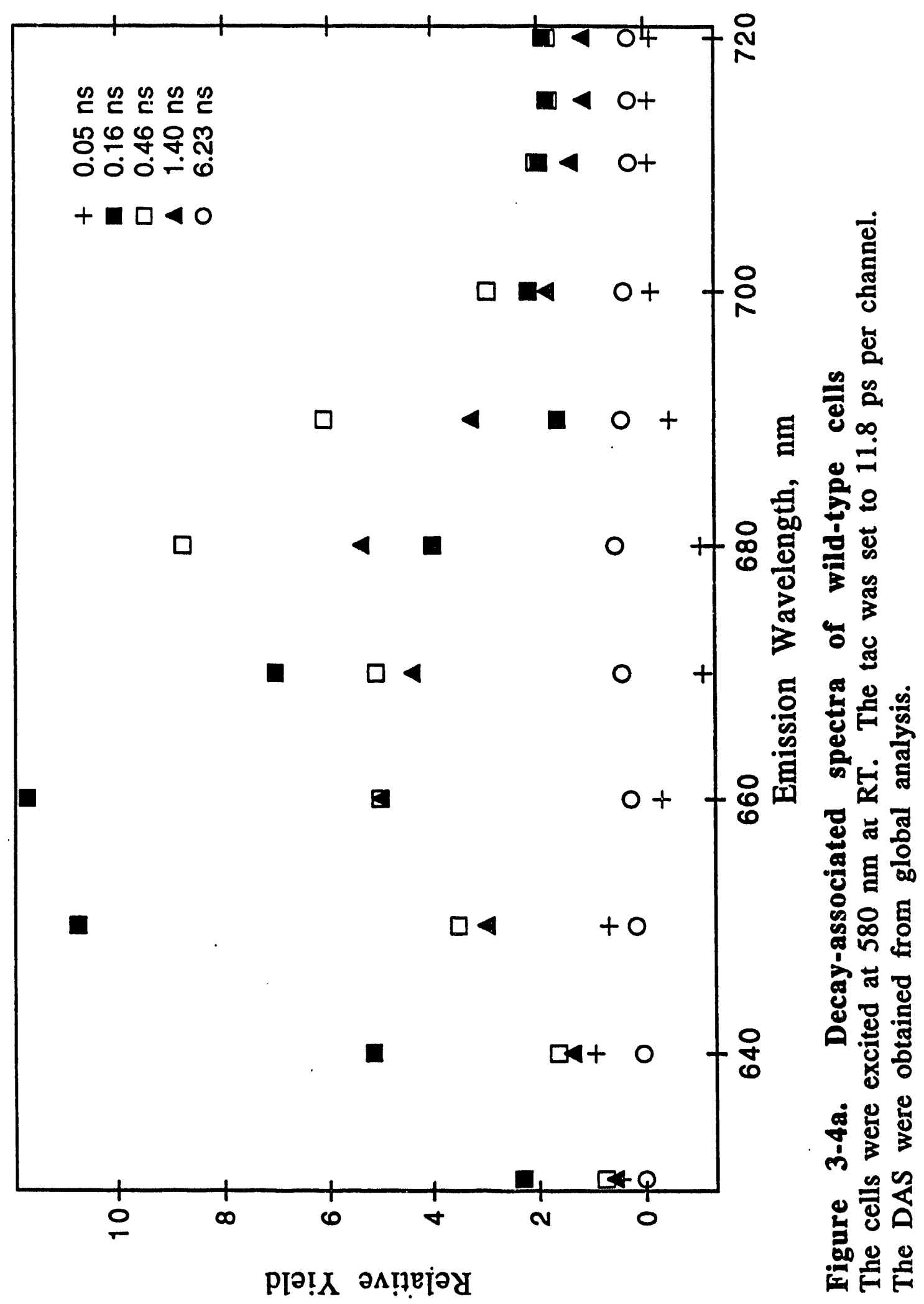




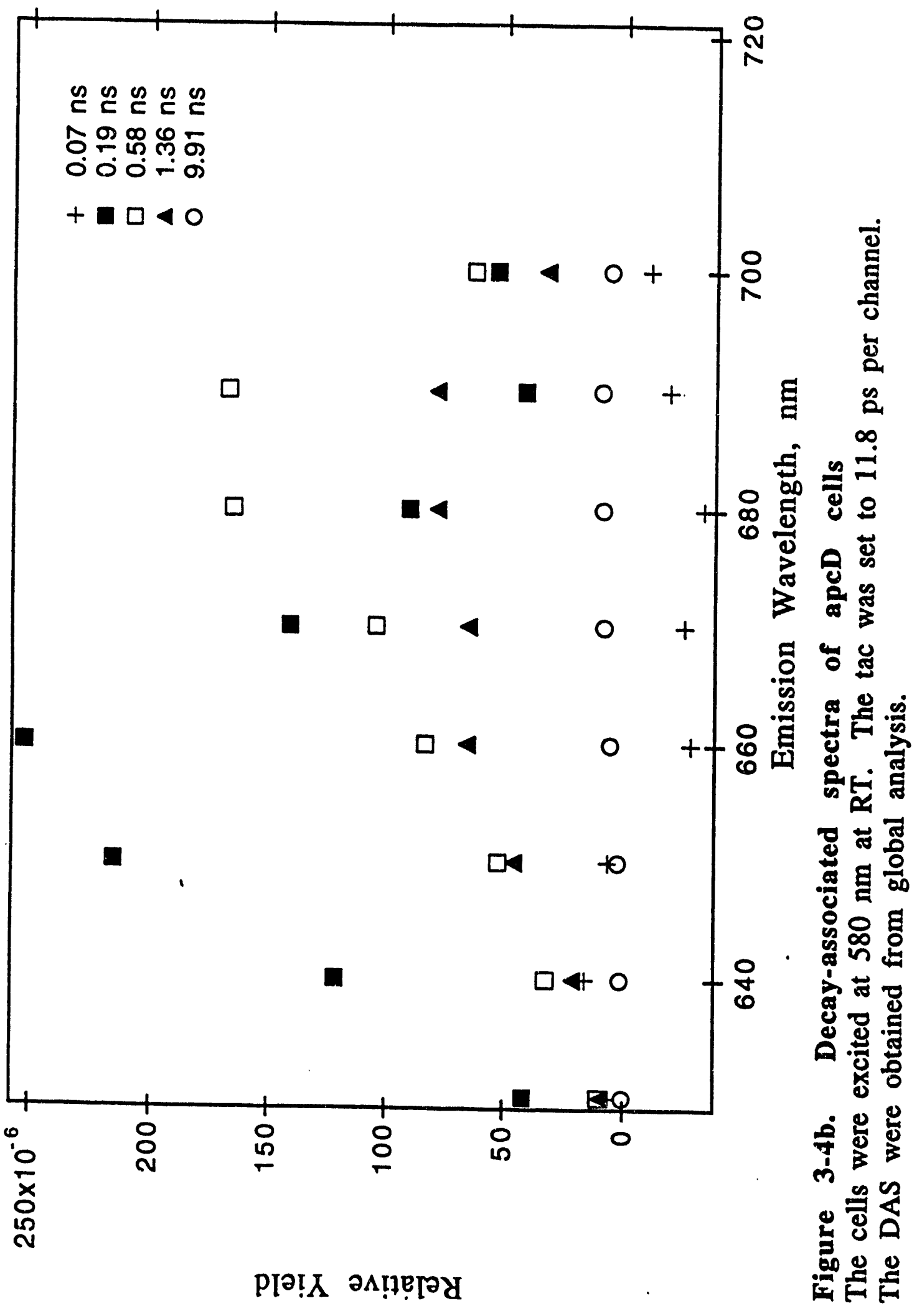




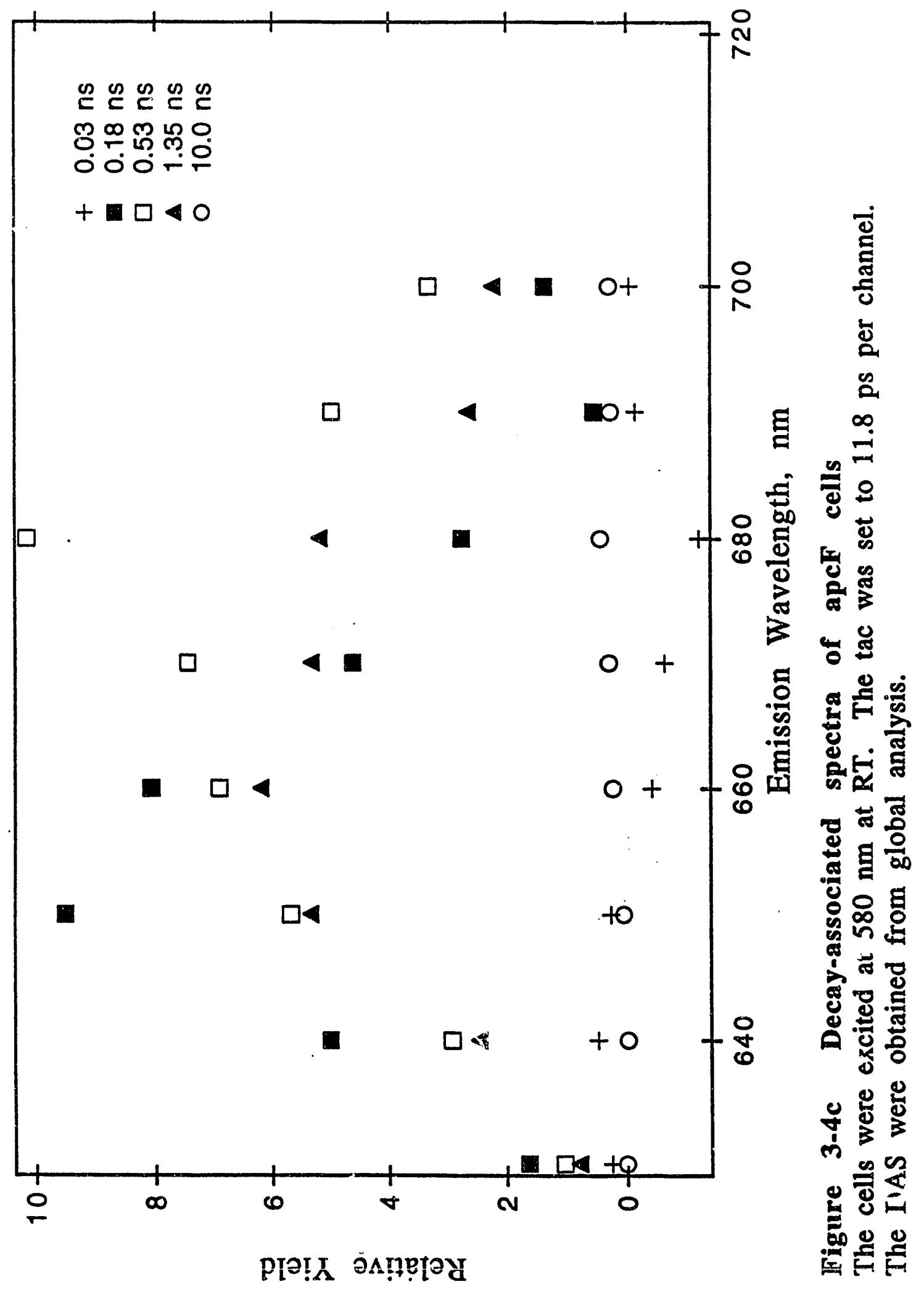




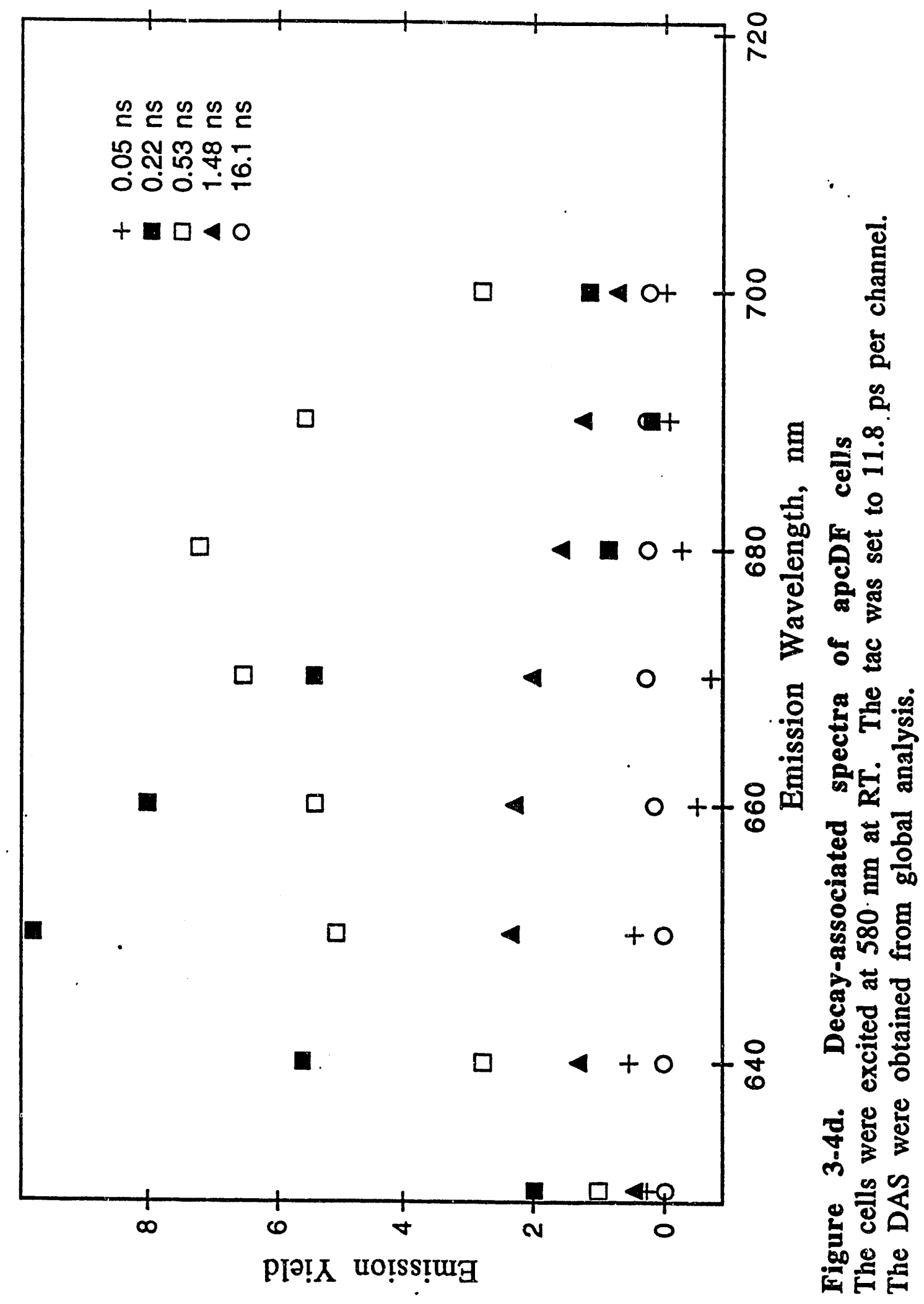




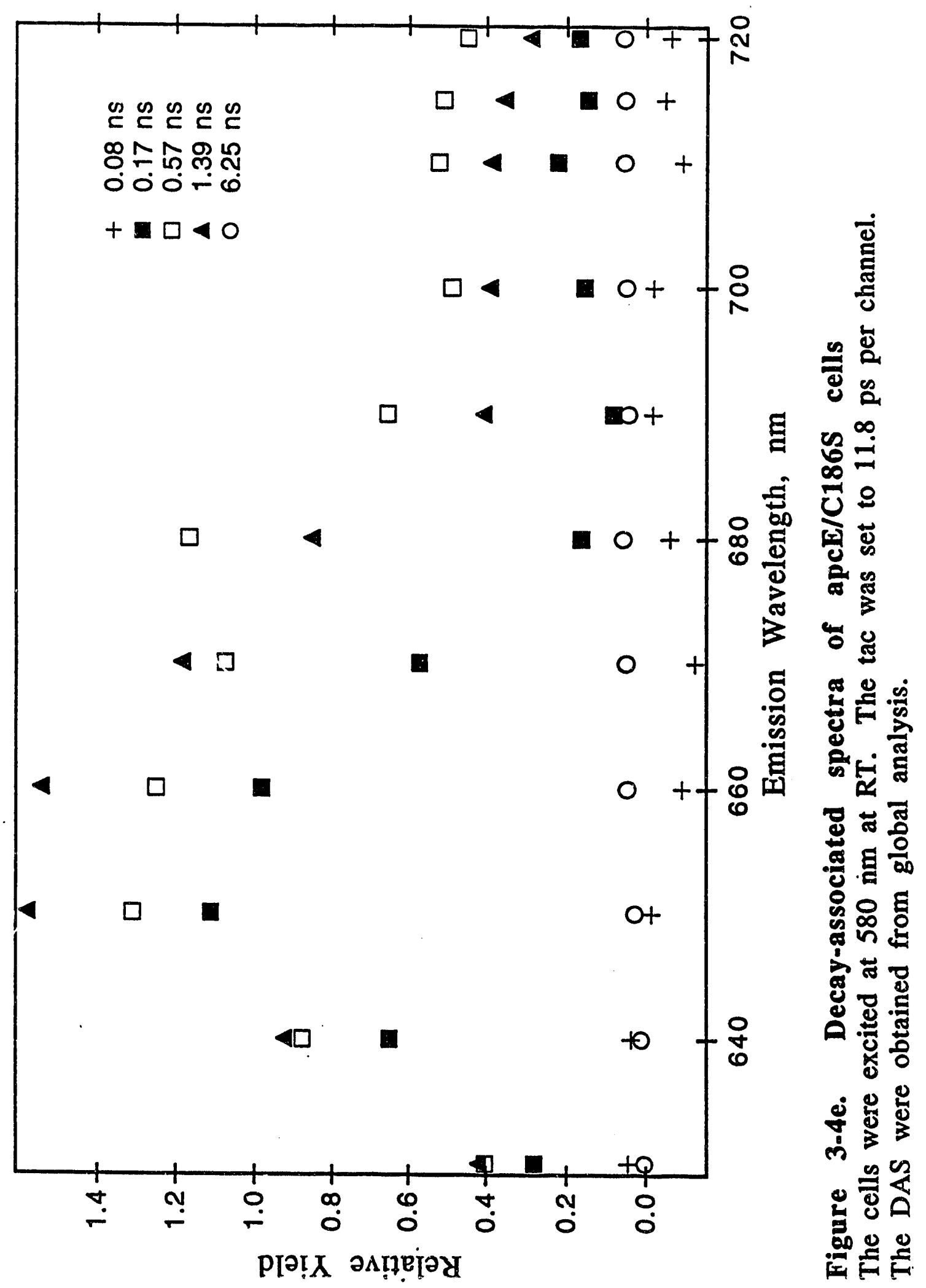




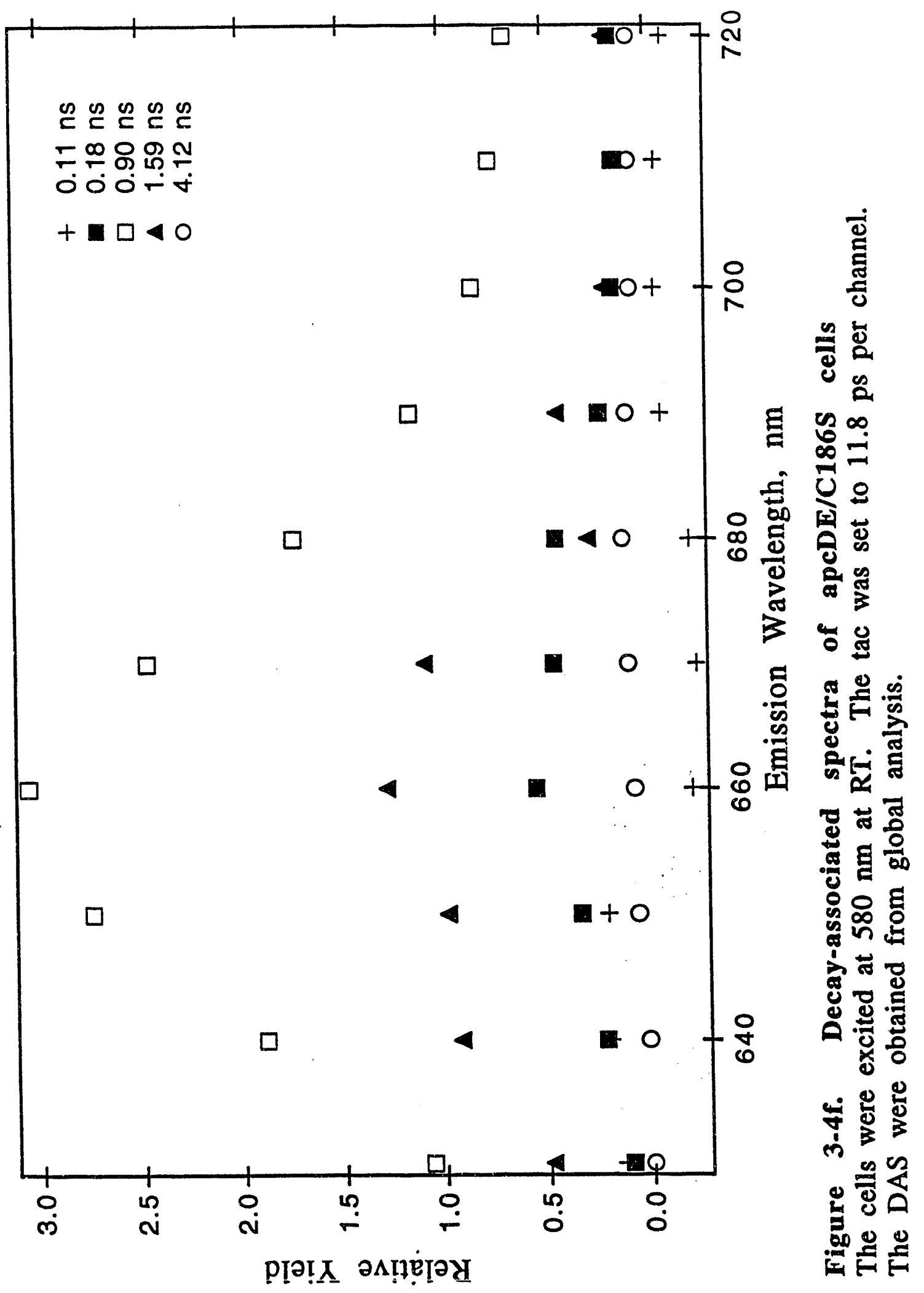


emission spectrum. The very long-lived component of $10 \mathrm{~ns}$ which contributes very little to the total yield is probably from free chl $a$.

The $0.5 \mathrm{~ns}$ component was also reported in all of the studies, and Mullineaux and Holzwarth (1991) attribute it to the PSII reaction center based on studies done with closed and open reaction centers. The remaining component in the wild-type DAS of $1.4 \mathrm{~ns}$ is assigned to both dissociated phycobiliprotein and to intact PBS uncoupled from the reaction centers. All of these assignments were made using the resolved emission spectra of the individual components along with the DAS spectra from isolated PBS shown in Chapter 4. For example, the $1.4 \mathrm{~ns}$ component, which is associated with emission from 640 to $690 \mathrm{~nm}$, is very similar to the $1.5 \mathrm{~ns}$ component found in the DAS for intact wild-type PBS.

Both Bittersmann and Mullineaux resolve a very fast component of $0.020 \mathrm{~ns}$ with very low yield, which they attribute to the PC pigment pool (Mullineaux and Holzwarth, 1991; Bittersmann and Vermaas, 1991). I was not able to resolve this component, possibly because I was collecting only one-fourth the number of counts per decay. Contributions from the PC pigment pool are included in the 0.05 ns component.

From the assignments I've described above, the most important components in the decays are the $0.16 \mathrm{~ns}$ and $0.05 \mathrm{~ns}$ components attributed to coupled chromophores within the intact PBS and the 1.4 ns component assigned to uncoupled phycobiliprotein.

As shown in Figure 3-4b,4c, and $4 d$, the DAS obtained for the apcD, apcF, and apcDF cells are indistinguishable from that of the wild-type within the resolution of the experiment. The $\mathrm{apcD}$, $\mathrm{apcF}$, 
and apcDF cells do not appear to contain any more free phycobiliprotein than the wild-type cells.

The DAS obtained for the apcE/C186S and apcDE/C186S cells are considerably different from that of the wild-type. In the apcE/C186S DAS, two risetimes are present: one at about $680 \mathrm{~nm}$ similar to that found in the wild-type DAS, and one at $710 \mathrm{~nm}$. The risetime at $710 \mathrm{~nm}$ is due to energy transfer to the modified chromophore from higher energy pigments. As with the steady state emission data, there is no evidence for the presence of the modified chromophore in the apcDE/C186S cells. The risetimes found at $680 \mathrm{~nm}$ for the apcDE/C186S and apcE/C186S cells are slightly longer than that found in the wild-type. Energy transfer from the outlying chromophores to the reaction centers or terminal emitters is not happening as efficiently in the apcDE/C186S and apcE/C186S mutants.

The apcE/C186S DAS displays a 0.57 ns component that emits at $680 \mathrm{~nm}$ and that can be attributed to the PSII reaction centers; the reaction center appears to function in the apcE/C186S cells. The apcDE/C186S DAS does not show any comparable component that can be assigned to the PSII reaction center. In fact, there is very little emission at $680 \mathrm{~nm}$ shown in the apcDE/C186S DAS. The only component in the apcDE/C186S DAS to show any appreciable fluorescence yield at $680 \mathrm{~nm}$ is a $0.9 \mathrm{~ns}$ component, which I attribute to intact uncoupled PBS.

The apcDE/C186S DAS does contain the 0.18 ns component, which is analogous to the $0.16 \mathrm{~ns}$ component that $\mathrm{I}$ attribute to chromophores within the PBS coupled to the reaction centers, but it 
is a much smaller percentage of the total yield of the cells. Apparently, energy transfer to the PSII reaction center from the apcDE/C186S PBS is considerably impaired.

Mullineaux et al. reported that closed reaction centers in Synechococcus 6301 cells had a longer fluorescence lifetime, $0.56 \mathrm{~ns}$ compared to $0.48 \mathrm{~ns}$ with open reaction centers (Mullineaux, Bittersmann et al., 1990). It seems unlikely that the lifetime of closed reaction centers would increase to $0.90 \mathrm{~ns}$ in Synechococcus sp. PCC 7002. I would rather attribute the $0.90 \mathrm{~ns}$ component in the apcDE/C186S DAS to uncoupled, intact PBS. In Chapter 4, I will show that apcDE/C186S PBS fluorescence decays with a $1.1 \mathrm{~ns}$ component. I find no trace of fluorescence from the PSII reaction center in the apcDE/C186S clata, but if it is a very small percentage of the total yield of the cell then I would not resolve the reaction center emission.

The other significant difference between the apcDE/C186S and apcE/C186S DAS and the wild-type DAS is the larger relative yields of components which can be assigned to either dissociated phycobiliproteins or uncoupled, intact PBS. In the apcE/C186S DAS, a 1.39 ns component which appears to be due to emission from a combination of both dissociated phycobiliprotein and uncoupled PBS contributes a much larger portion of the total fluorescence yield compared to the wild-type DAS. The apcDE/C186S DAS has a 1.59 ns component which I attribute to dissociated phycobiliprotein and a 0.90 ns component which I assigned to uncoupled PBS. The shorter component is due to the chromophores coupled to the modified chromophore present in the apeDE/C186S PBS, as I will 
show in Chapter 4. These two decay components contribute almost all of the emission present in the apcDE/C186S cells.

\section{Conclusions}

There are no significant differences in the ratio of phycobiliprotein to chl $a$ concentrations found in the different strains as shown in the absorption spectra. None of the mutants appear to make larger amounts of $\operatorname{chl} a$ to compensate for impaired PBS.

The $a p c D$, apcF, and apcDF strains were indistinguishable from the wild-type using steady state and time-resolved fluorescence spectroscopy. Cells from these mutants have similar quantum yields along with similar concentrations of free phycobiliprotein and uncoupled PBS. The PBS from these mutant transfer energy to $680 \mathrm{~nm}$ emitters as efficiently as the wild-type PBS. This conclusion is consistent with the doubling times measured for the apcD, apcF, and apcDF mutants, as shown in Table 2-2. The mutants all had doubling times similar to that of the wild-type strain.

Cells from the apcDE/C186S and apcE/C186S strains display different steady state and time-resolved emission spectra from the wild-type cells. These mutants contain larger amounts of dissociated phycobiliprotein and uncoupled PBS along with PBS that display impaired energy transfer to the $680 \mathrm{~nm}$ emitters (both the reaction centers and the terminal emitters from the PBS). All of these factors contribute to the greater fluorescence yield of cells from the apcE/C186S and apcDE/C186S mutants relative to the wild-type. 
Energy transfer from the PBS to the PSII reaction center occurs in the apcE/C186S cells but only on a small scale in the apcDE/C186S cells. None of the resolved components in the apcDE/C186S DAS could be attributed to the reaction center. From the time-resolved fluorescence data, the apcDE/C186S cells appear to be especially impaired. Only small amounts of energy are transferred to the PSII reaction centers from the antenna.

Both the DAS and steady state emission spectra for the apcE/C186S cells show the presence of a modified chromophore that emits at approximately $710 \mathrm{~nm}$. The modified chromophore emission does not appear to be present in the apcDE/C186S spectra. Energy transfer from PC and AP in the antenna to the modified chromophore occurs at the same rate as energy transfer to the 680 $\mathrm{nm}$ emitters, as shown by the risetimes at 710 and $680 \mathrm{~nm}$ in the apcE/C186S DAS.

The doubling time of the apcE/C186S strain is five times that of the wild-type strain. The impaired growth of the apcE/C186S strain can be attributed to three things. First, the apcE/C186S cells contain more dissociated phycobiliproteins and uncoupled PBS which the cells waste energy making. Second, energy transfer is less efficient in the PBS that are coupled to the reaction centers. Third, energy that would normally carry out photochemistry is being funneled away from the PSII reaction centers to the modified chromophore to be lost as fluorescence.

I don't have a doubling time for the apcDE/C186S strain from Bryant for the conditions given in Table 2-2, but I know that this strain grows at a significantly slower rate. From my emission data I 
attribute the slower growth rate to be due to the decrease in energy transfer from the PBS to the reaction centers. The apcDE/C186S cells also appear to contain more dissociated phycobiliproteins.

One question which I haven't address yet is what is the composition of the PBS from the mutants. The apcD PBS is missing the $\alpha^{A P-B}$ subunit, the apcF PBS is missing the $\beta^{18}$ subunit, and the apcDF PBS is missing both $\alpha^{A P-B}$ and $\beta^{18}$. What happens in the PBS core when polypeptides are missing? Do water molecules just fill the space, or does a polypeptide similar in amino acid composition substitute for the missing protein? In the next chapters I will present data which indicate that a bluer-absorbing protein substitutes for the $\beta^{18}$ polypeptide, and probably the $\alpha^{A P-B}$ protein also. I suggest that an $\alpha^{\mathrm{AP}}$ subunit replaces the $\alpha \mathrm{AP}-\mathrm{B}$ subunit and a $\beta^{A P}$ subunit replaces the $\beta^{18}$ subunit. This suggestion is based on the similarity between the amino acids sequences for the $\alpha \mathrm{AP}-\mathrm{B}$ and $\alpha \mathrm{AP}$ proteins and the $\beta^{18}$ and $\beta^{\mathrm{AP}}$ proteins (Glazer and Bryant, 1975; Füglistaller, Rümbeli et al., 1984). Since the proteins have similar amino acids sequences, it seems reasonable to assume that their structures will also be similar. Therefore, the proteins are possibly interchangeable to a certain extent within the PBS core. I will discuss this point further in the next chapters.

The apcDE/C186S and apcE/C186S mutants did not turn out as originally expected. The substitution of a serine for the chromophore-binding cysteine on the $\mathrm{L}_{\mathrm{cm}}$ was expected to cause the deletion of the chromophore from the polypeptide, but this obviously did not occur. The chromophore is present in a modified form. It absorbs and emits light at lower energies than the 
chromophore normally bound to the $\mathrm{L}_{\mathrm{cm}}$ protein, as I will show in the following chapters.

It seems reasonable to assume that the modified chromophore is being held noncovalently in the binding pocket of the $\mathrm{L}_{\mathrm{cm}}$ polypeptide. The spectral properties of the modified chromophore is more similar to that of the bound chromophores found in the phycobiliproteins then to the spectral properties of the free chromophore (Braslavsky, Holzwarth, et al., 1983). As I will show in the next chapter, emission from the modified chromophore decays with a time constant of approximately 1 ns. Braslavsky et al. found that the emission from biliverdin dimethyl esters decayed with lifetimes of less than 50 ps.

I have found that the modified chromophore is easily lost from the PBS core when the PBS is partially dissociated, but it seems relatively stable within the intact PBS. The modified chromophore is possibly $3(\mathrm{Z})$-phycocyanobilin, the immediate precursor to the bound pigment as reported by Rhie and Beale (Rhie and Beale, 1992). 3(Z)-phycocyanobilin has a double bond on the carbon that, in the bound chromophore, binds to the cysteine group of the protein.

The addition of this extra double bond in the modified chromophore makes it more conjugated than the bound pigment found on the wild-type $\mathrm{L}_{\mathrm{cm}}$. The increased conjugation decreases the energy gap between the lowest excited state of the molecule and the ground state, thus shifting the absorption spectrum of the chromophore further to the red. I will discuss spectral properties of the modified chromophore more in the next chapter. 
References for Chapter 3

Bittersmann, E. and W. Vermaas (1991). Biochim. Biophys. Acta 1098: $105-116$.

Braslavsky, S. E., A. R. Holzwarth, and K. Schaffner (1983). Angew. Chem. Int. Ed. Engl. 22: 656-674.

Bryant, D. A. (1991). Cyanobacterial Phycobilisomes: Progress toward Complete Structural and Functional Analysis via Molecular Genetics, In L. Bogorad and I. K. Vasil (eds.), Cell Culture and Somatic Cell Genetics of Plants., Vol 7B, Academic Press, Inc., Orlando, FL 257-300.

de Lorimier, R. M., R. L. Smith and S. E. Stevens, Jr. (1992). Plant Physiol. 98: 1003-1010.

Füglistaller, P.,R. Rümbeli, F. Suter, and H. Zuber (1984). HoppeSeyler Z Physiol Chem, 365: 1085-1096.

Glazer, A. N. and D. A. Bryant (1984). Arch. Microbiol. 104: 15-22.

Mullineaux, C. W., E. Bittersmann, J. F. Allen and A. R. Holzwarth. (1990). Biochim. Biophys. Acta 1015: 231-242.

Mullineaux, C. W. and A. R. Holzwarth (1991). Biochim. Biophys. Acta 1098: 68-78.

Myers, J., J. R. Graham and R. T. Wang (1980). Plant Physiol. 66: 1144-1149.

Rhie, G. and S. I. Beale (1992). J. Biol. Chem. 267: 16088-16093.

Stevens, S. E., Jr., D. L. Balkwill and D. A. M. Paone (1981). Arch. Microbiol. 130: 204-212. 


\section{Chapter 4. Discussion of PBS Results}

In the last chapter the spectroscopy of the whole cells was presented to examine how the mutations in the PBS affected energy transfer to the reaction centers. In this chapter, I will explore the spectral properties of the isolated intact PBS. I am making the assumption that the isolated PBS have spectral properties identical to PBS in the cells except that the terminal emitters in the PBS core should now simply fluoresce instead of transferring energy to chl $a$.

The PBS were easily isolated on sucrose density gradients as discussed in Chapter 2. The amount of free phycobiliprotein present on the gradient increased in order of wild-type < apct, apcD, apcDF < apcE/C186S < apcDE/C186S. I had some problems isolating intact PBS from the apcDE/C186S mutant; most of the phycobiliprotein isolated on the sucrose gradient was dissociated into smaller structures such as rods or hexamers.

I showed in the previous chapter that the amount of dissociated phycobiliprotein present in the intact cells for the $\mathrm{apcD}$, apcF, and apcDF mutants was similar to that of the wild-type while the apcE/C186S and apcDE/C186S cells contained more dissociated phycobiliproteins and/or uncoupled PBS. It also appears that the PBS from the $a p c D, a p c F$, and apcDF mutants are more unstable under the conditions used for their isolation than the wila-type PBS. I diluted concentrated PBS removed from the gradients using $0.75 \mathrm{M}$ phosphate buffer at $\mathrm{pH} 7.8$ with $1.0 \mathrm{M}$ sucrose. The addition of sucrose to the dilution buffer increased the stability of 
the preparations, and the change in solvent did not affect the emission spectrum obtained from the PBS.

\section{Absorption}

I compared the absorption spectra of PBS from the wild-type and mutant strains to quantify changes in the composition of the PBS with each mutation. I make the assumption initially that the absorbances of the phycobiliproteins are additive. In other words, the mutations have not affected the absorption of other proteins in the PBS core. None of the missing chromophores are involved in excitonic coupling. For example, the absence of the $\beta^{18}$ subunit does not affect the absorption spectrum of an adjacent AP monomer in the trimer. If this assumption is correct, the differences that I expect are small, on the order of 0.005 a.u. in the normalized spectrum.

The absorption spectra of the PBS are given in Figures 4-1a,1b. All of the spectra have absorption maxima at $630 \mathrm{~nm}$. The apcDE/C186S, apcE/C186S and apcDF PBS absorb slightly less at approximately $600 \mathrm{~nm}$ and above $650 \mathrm{~nm}$. The apcD and apcF PBS are virtually indistinguishable from the wild-type.

Small variations in the $600 \mathrm{~nm}$ absorption are possibly due to changes in PC content or to differences in core composition. As I mentioned earlier, the amount of PC in the rods can vary somewhat with light conditions (de Lorimier, Smith et al., 1992), and these changes will appear in the absorption spectrum. The absorption spectrum of AP trimers also contains a shoulder at $600 \mathrm{nnin}$, so the changes in the PBS core composition will influence the AP 


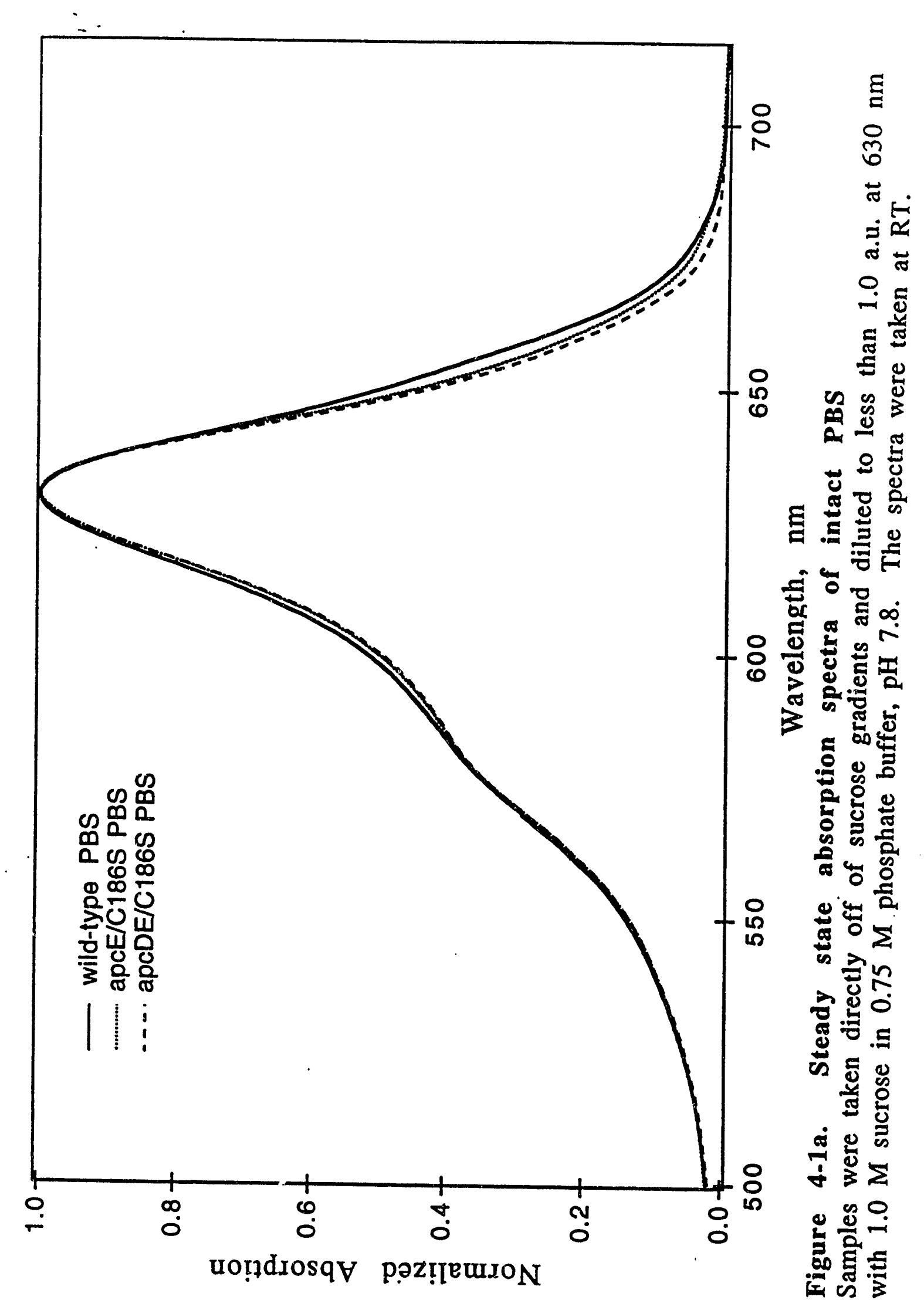




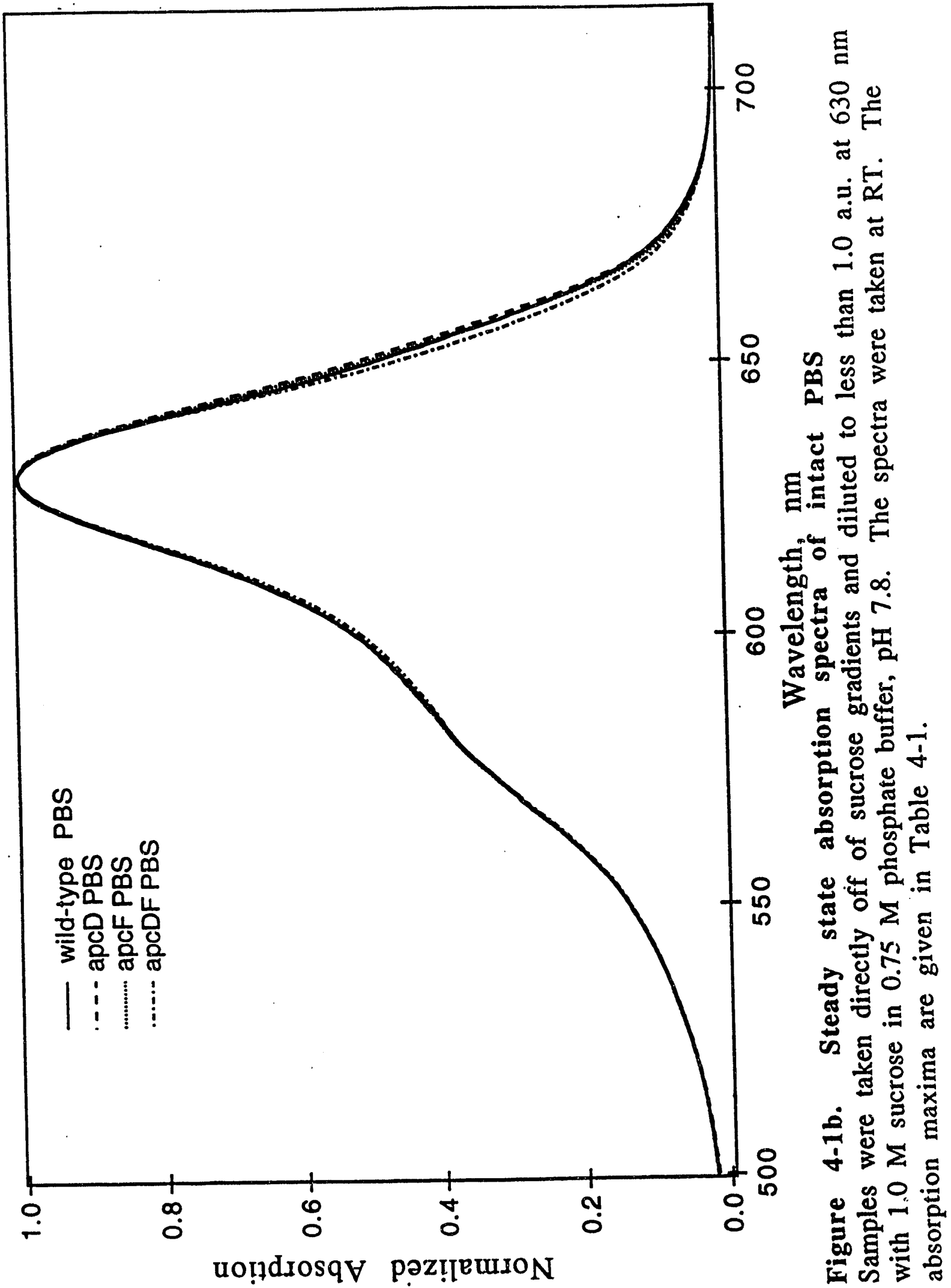


absorption shoulder at $600 \mathrm{~nm}$ along with the absorption maximum at $650 \mathrm{~nm}$.

I calculated absorption difference spectra for all of the mutants by normalizing the area under the absorption curves and then subtracting the absorption of the PBS from the mutant from that of the wild-type PBS, as shown in Figures 4-2a,2b. With this subtraction procedure I make the assumption that the number of chromophores present is unchanged from wild-type to mutant. The net change in absorption has to be zero with this procedure.

The $\mathrm{L}_{\mathrm{cm}}$ and $\alpha$ AP-B chromoproteins have been assigned absorption maxima at $675 \mathrm{~nm}$ (Mimuro, Yamazaki et al., 1989; Lundell, Yamanaka et al., 1981; Glazer and Bryant, 1975). AP trimers absorb at $650 \mathrm{~nm}$ with a $600 \mathrm{~nm}$ shoulder, while PC hexamers absorb at about $625 \mathrm{~nm}$. If the absorption spectra of the chromophores are additive, the absence of specific chromophores will leave holes in the absorption spectra of the PBS from the mutants. The holes should appear in the difference spectra.

The difference spectra for the apsE/C186S, apcDE/C186S, and apcDF mutants are very similar in appearance. The PBS from these mutants absorb more than the wild-type at 580 and $630 \mathrm{~nm}$ and less than the wild-type at $655 \mathrm{~nm}$ with a definite shoulder at approximately $675 \mathrm{~nm}$. The apcDE/C186S and apcE/C186S PBS also absorb more than the wild-type PBS at $695 \mathrm{~nm}$. The apcD and apcF difference spectra are almost reversed in appearance from the other spectra; the apcD and apcF PBS absorb more than the wildtype at approximately $650 \mathrm{~nm}$, and less than the wild-type at 


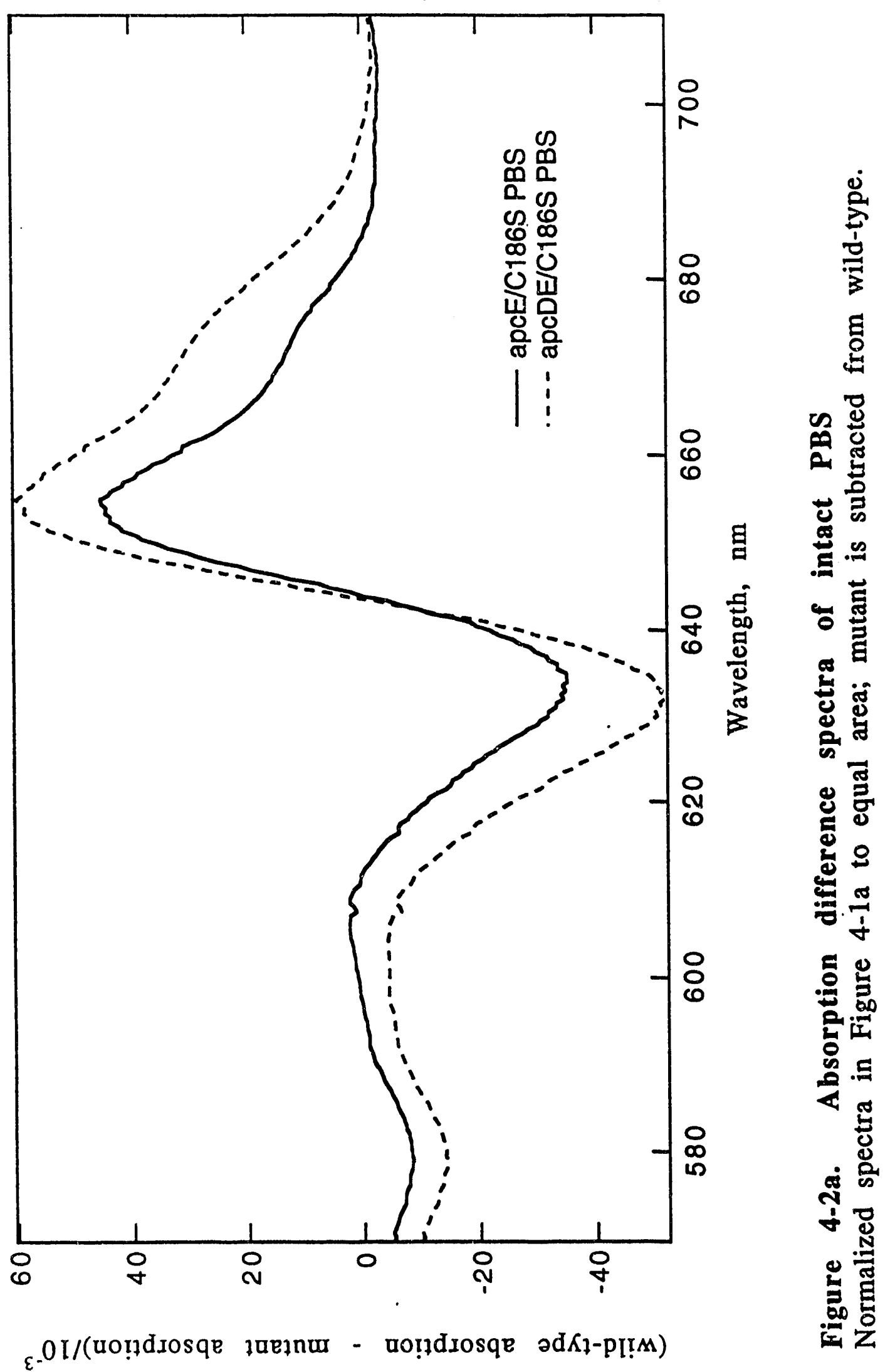




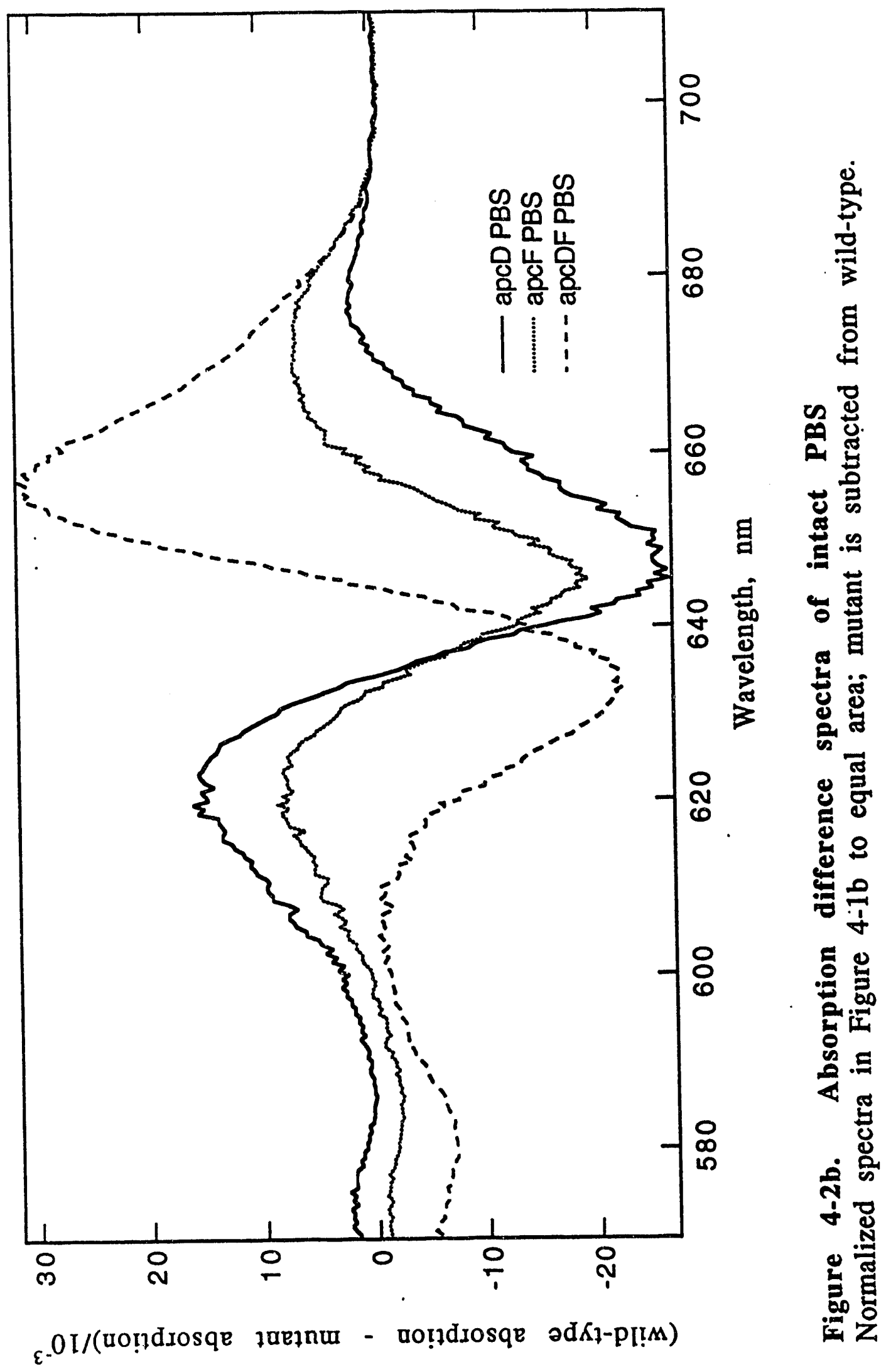


$630 \mathrm{~nm}$ and $670 \mathrm{~nm}$. The apcDE/C186S and apcE/C186S absorption differences are approximately twice that of the other mutants.

I tried several other ways to execute the absorption subtraction. I normalized the spectra at several different wavelengths, and I tried a subtraction method in which the wildtype absorption is slightly greater than the absorption of the PBS from the mutants. None of the methods gave consistent results for the wavelength region from 600 to $650 \mathrm{~nm}$ for any of the strains. The various difference spectra calculated for the apcDE/C186S and apcE/C186S PBS consistently showed that the apcDE/C186S and apcE/C186S PBS absorb more than the wild-type at about 695 and less than the wild-type PBS at about 675. The smaller absorption differences in the rest of the mutants were niore easily distorted by the subtraction procedures; I don't feel confident about any of the differences in the $a p c F$, apcD, and apcDF absorption spectra.

\section{Steady State Fluorescence}

Steady state emission spectra were obtained for all of the strains, as shown in Figures 4-3a,3b. Quantum yields were measured for the PBS from the mutants relative to that of the wildtype, as shown in Table 4-1. The emission spectra of the PBS should enable us to judge the energy transfer efficiency of the isolated antenna. If the chromophores in PBS are not as well coupled, they won't transfer energy as efficiently to the terminal emitter(s). More emission will be seen from PC and AP chromophores. Energy transfer won't compete as well with fluorescence deactivation as in the wild-type antenna. 


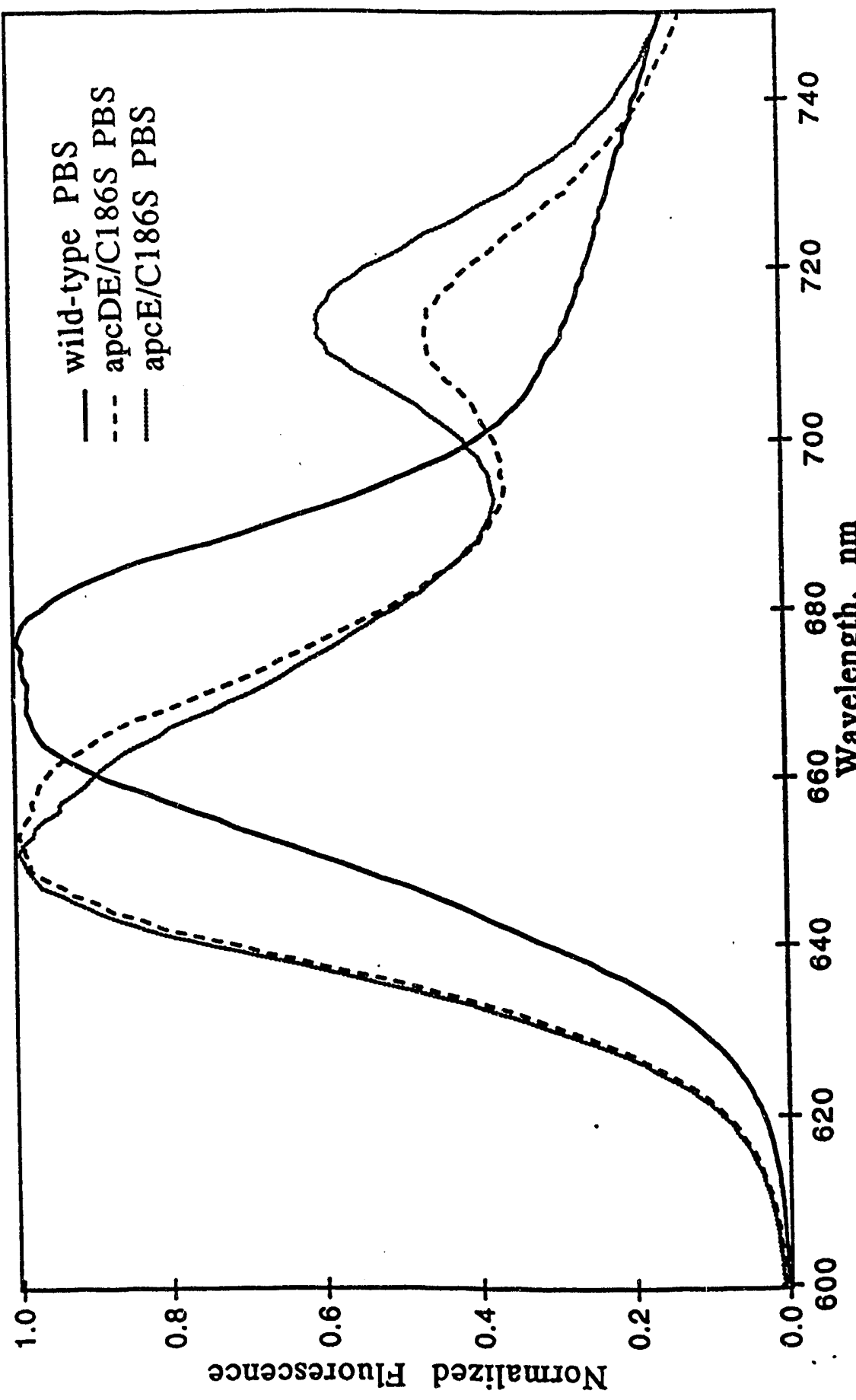

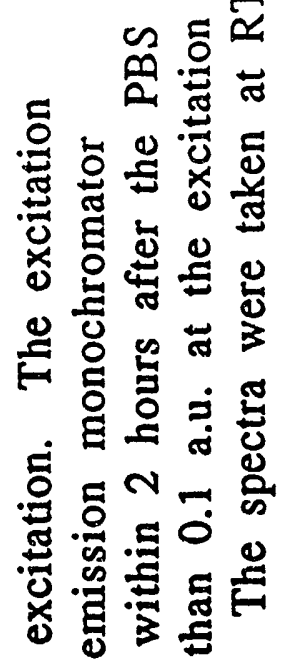

冓

- 요원

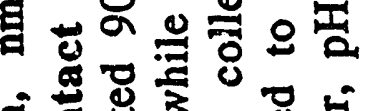

চ艹

엉 응융

๘

हैं क्ष

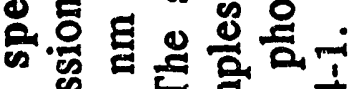

- $m$ E

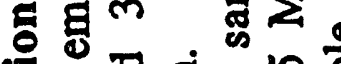

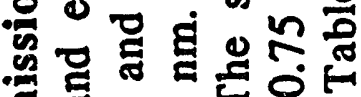

ह

- $00^{\infty} .5 . \Xi$

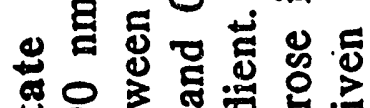

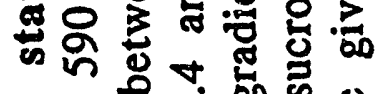

$\Rightarrow \pi 00$ क क

"ृष

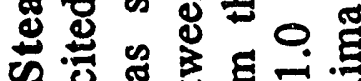

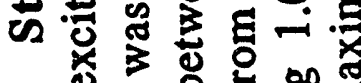
- 仓 ஸे थ 은 등 행.

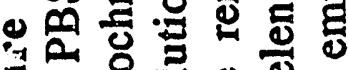
스웡

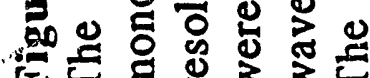

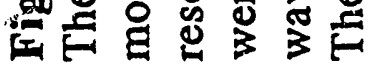




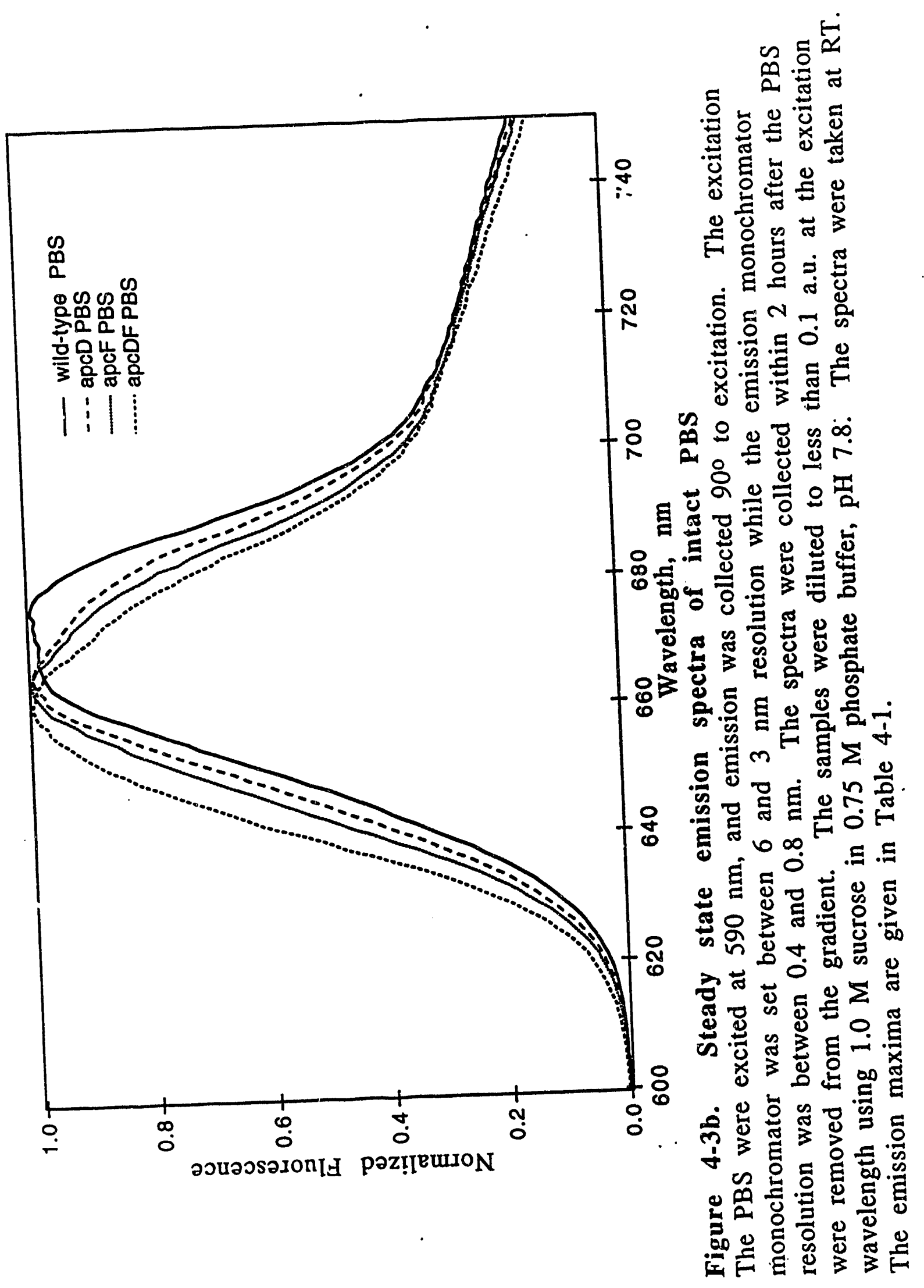


Table $4-1$

Spectral Data on Intact Phybilisomes

\begin{tabular}{cccccc} 
& $\begin{array}{c}\text { Absorption } \\
\text { Maximum }\end{array}$ & $\begin{array}{c}\text { RT } \\
\text { Emission } \\
\text { Maximum }\end{array}$ & $\begin{array}{c}77 \mathrm{~K} \\
\text { Quantum } \\
\text { Yield" }\end{array}$ & $\begin{array}{c}\text { Emission } \\
\text { Maximum }\end{array}$ \\
\cline { 2 - 2 } & Wild-Type & $630 \mathrm{~nm}$ & $676 \mathrm{~nm}$ & 100 & $683 \mathrm{~nm}$ \\
apcF & $630 \mathrm{~nm}$ & $665 \mathrm{~nm}$ & $102 \pm 12$ & $681 \mathrm{~nm}$ \\
apcD & $630 \mathrm{~nm}$ & $666 \mathrm{~nm}$ & $108 \pm 8$ & $683 \mathrm{~nm}$ \\
apcDF & $630 \mathrm{~nm}$ & $661 \mathrm{~nm}$ & $104 \pm 11$ & $681 \mathrm{~nm}$ \\
apcE/C186S & $630 \mathrm{~nm}$ & $651 \mathrm{~nm}$ & $54 \pm 5$ & $682 \mathrm{~nm}$ \\
& & & $714 \mathrm{~nm}$ & & $715 \mathrm{~nm}$ \\
apcDE/C186S & $630 \mathrm{~nm}$ & $653 \mathrm{~nm}$ & $52 \pm 14$ & $678 \mathrm{~nm}$ \\
& & & $712 \mathrm{~nm}$ & & $713 \mathrm{~nm}$
\end{tabular}

\#Quantum yields were calculated at room temperature relative to wild-type PBS. The PBS were excited at $590 \mathrm{~nm}$, and the emission from 600 to $800 \mathrm{~nm}$ was integrated to give the relative yield. The numbers are given in percentage of wild-type yield. A minimum of three measurements were averaged for each strain to give the standard deviation. 
The appearance of more AP and PC emission could also be due to a change in population distribution because fewer $680 \mathrm{~nm}$ emitters are present. Assuming a Boltzmann distribution of populations, more emission will originate from the higher energy states if the number of lower energy states decreases. This conclusion especially holds at room temperature where back transfer is important. Therefore, AP and PC emission will increase in the mutants missing $680 \mathrm{~nm}$ fluorescers.

The steady state emission spectra are shown in Figures $4-3 a, 3 b$. I was interested in seeing the effect of the decrease in the number of terminal emitters on the steady state emission spectrum of the PBS from the mutants. I used the following formula to calculate the relative Boltzmann populations, where $n_{i}$ is the fraction of $i$ type chromophores, $g_{i}$ is the number of $i$ type chromophores, and $\Delta E$ is the energy difference between the $E_{o}$ levels of the chromophores:

$$
\frac{n_{i}}{n_{j}}=\frac{g_{i}}{g_{j}} e^{(-\Delta E / k T)}
$$

The $\mathrm{E}_{\mathrm{o}}$ levels used corresponded to the average of the absorption and emission maxima.

For these calculations I make the assumption that the $\beta^{18}$ chromophore emits at $682 \mathrm{~nm}$ along with the $\alpha$ AP-B and $L_{c m}$ chromoproteins, that the $\beta 18$ and $\alpha$ AP-B subunits are replaced by $\beta \mathrm{AP}$ and $\alpha \mathrm{AP}$ subunits (this assumption had very little effect on the calculations), and that the chromophores are coupled. For the degeneracy of the energy states, I assumed that the wild-type PBS are composed of 324 PC chromophores, 46 AP chromophores, and 6 
terminal emitters. I made some gross assumptions concerning the energy levels of the pigments, because I did not take into account any differences in the spectral properties of chromophores from the same pigment pool. The $E_{0}$ energies used corresponded to $635 \mathrm{~nm}$ for $\mathrm{PC}, 656 \mathrm{~nm}$ for $\mathrm{AP}, 679 \mathrm{~nm}$ for the $680 \mathrm{~nm}$ emitters, and 705 $\mathrm{nm}$ for the modified chromophore present in apcDE/C186S and apcE/C186S PBS. The changes in the emission spectrum from mutant to wild-type PBS are calculated to occur in the absence of any structural perturbations that affect chromophore coupling.

For wild-type PBS, I calculate that $19 \%$ of the steady state emission (emission after equilibration) will originate from PC, $32 \%$ from AP, and $49 \%$ from the $680 \mathrm{~nm}$ emitters. For the apcD and apcF mutants, the calculated percentage of emission from each chromophore type should be $23 \%$ from PC, $38 \%$ from AP, and $39 \%$ from the $680 \mathrm{~nm}$ emitters while the apcDF emission contributions will be $28 \%, 49 \%$, and $23 \%$, respectively.

The calculations for the wild-type emission spectrum qualitatively fit the experimental data. With the decrease in the number of $680 \mathrm{~nm}$ emitters present, the Boltzmann calculations predict a blue-shift in the steady state spectrum for the mutants. This prediction qualitatively fits the experimental data on the apcD, apcF, and apcDF mutants. The emission from the apcDF mutant is shifted further blue than the other mutants as expected.

The Boltzmann calculations did not work well for the apcDE/C186S or apcE/C186S PBS. About $75 \%$ of the emission should be coming from the modified chromophore, but this is obviously not 
the case, as shown in Figure 4-3a. I fit the 650 and $715 \mathrm{~nm}$ emission bands in the apcE/C186S and apcDE/C186S spectra to gaussians to calculate the relative yields of each band. The $715 \mathrm{~nm}$ band contributes only about one-third of the total yield in the apcDE/C186S and apcE/C186S PBS. In the apcDE/C186S and apcE/C186S PBS, most of the emission is originating from PC chromophores.

The emission spectra for the apcDE/C186S and apcE/C186S PBS show a virtual absence of $680 \mathrm{~nm}$ emission along with a strong emission band at $715 \mathrm{~nm}$ attributed to the modified chromophore. Boltzmann calculations indicate that less than $10 \%$ of the total emission in the steady state emission spectrum should be from the $680 \mathrm{~nm}$ emitters in the apcDE/C186S and apcE/C186S mutants if all the chromophores are coupled. The $\beta^{18}$ and $\alpha$ AP-B chromophores should transfer energy to the $715 \mathrm{~nm}$ emitter.

I examined the relative quantum yields of the PBS to see if more structural disorganization is present in the PBS from the mutants. If the chromophores are not being held in optimal conformations, more nonradiative deactivation could occur, and the quantum yield of the antenna will decrease. The relative quantum yields are given in Table 5-1. The yields obtained for the apcD, apcF, and apcDF PBS are essentially identical to that of the wild-type, while the apcE/C186S and apcDE/C186S yields are only one-half that of the wild-type. 


\section{Low Temperature Emission}

At $77 \mathrm{~K}$ the transfer of energy from the $680 \mathrm{~nm}$ emitters to AP or transfer from AP to PC is minuscule. The emission bands from PC, AP, and terminal emitters can be resolved; the populated excited states at $77 \mathrm{~K}$ are smaller in number than at $\mathrm{RT}$, and the emission bands will narrow. If the chromophores are coupled, the only emission that should be present is that from the terminal emitters.

From the $77 \mathrm{~K}$ emission spectra, I am able to make some spectral assignments for some of the terminal emitters. In the apcDF mutant only one of the three possible terminal emitters is present, the $\mathrm{L}_{\mathrm{cm}}$ chromophore. In the apcDE/C186S mutant only one $680 \mathrm{~nm}$ emitter is found, the $\beta^{18}$ chromophore, although the lower energy modified $\mathrm{L}_{\mathrm{cm}}$ chromophore is still present.

I make the assumption that the emission spectra of the chromophores in the PBS are not affected by the freezing process. I was successful in obtaining cracked glass samples at $77 \mathrm{~K}$ using $2 \mathrm{M}$ sucrose as a cryoprotectant. The emission spectrum of the samples before freezing and after rewarming were very similar. It does not appear that the freezing process caused any irreversible disruption of the antenna complex. I also found that the low-temperature emission spectra were very reproducible from experiment to experiment; the emission maxima for different preparations were within $0.5 \mathrm{~nm}$ of the maxima given in Table 4-1.

The PBS were excited at $590 \mathrm{~nm}$, directly exciting mostly PC in the rods. The experimental setup did not allow for the calculation of guantum yields, so only normalized spectra are present in 
Figures 4-4a,4b. I have also collected low temperature emission spectra for AP trimers and PC hexamers (not shown). AP trimers have a emission maximum at $659 \mathrm{~nm}$ while PC hexamers emit at $651 \mathrm{~nm}$. As I mentioned earlier, the wild-type emission spectrum has only one emission band present, at approximately $683 \mathrm{~nm}$. The rate of back transfer has slowed enough that emission from only the lowest energy chromophores occurs.

The emission spectra obtained for the apcDE/C186S and apcE/C186S PBS, shown in Figure $4-4 a$, are vastly different from that of the wild-type PBS. Instead of the one emission band present at $683 \mathrm{~nm}$ seen in the wild-type spectrum, the apcDE/C186S and apcE/C186S spectra contain three emission bands at approximately $645 \mathrm{~nm}, 680 \mathrm{~nm}$, and $715 \mathrm{~nm}$. The $645 \mathrm{~nm}$ band is due to PC, and the $715 \mathrm{~nm}$ band is from the modified chromophore. The $680 \mathrm{~nm}$ band is from $\beta 18$ emission in the apcDE/C186S mutant and $\beta^{18}$ and/or $\alpha$ AP-B emission in the apcE/C186S mutant.

There is a relative increase in the amount of PC emission compared to $680 \mathrm{~nm}$ emission in the apcDE/C186S and apcE/C186S emission spectra. I don't know whether the absolute amount of PC emission increases in the PBS from the mutants because the quantum yields couldn't be calculated.

The apcD, apcF, and apcDF low-temperature emission spectra are similar to that of the wild-type as shown in Figure 4-4b. The apcD emission spectrum matches the wild-type spectrum very well except for slightly less emission at about $690 \mathrm{~nm}$. The apcF and 


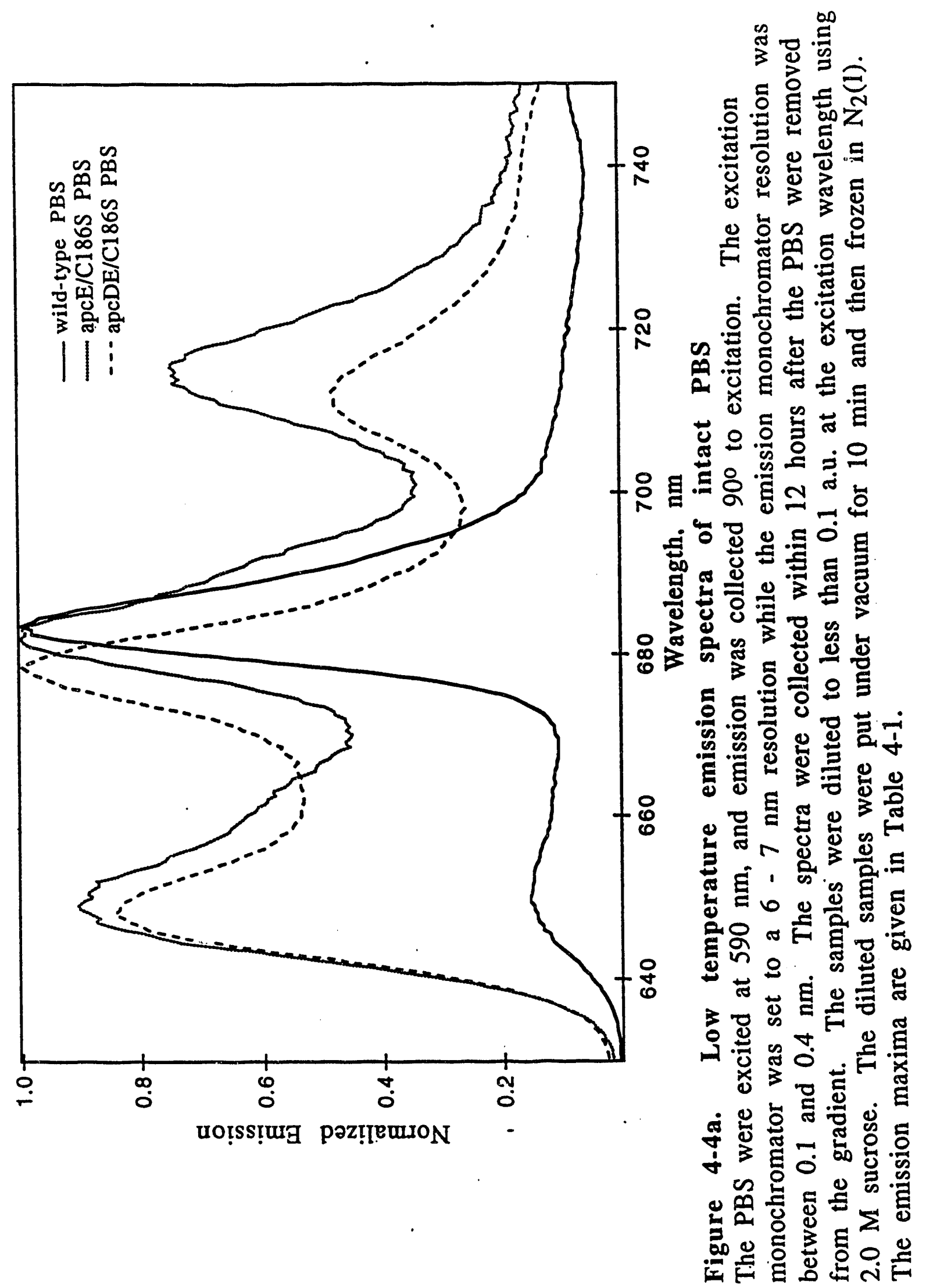




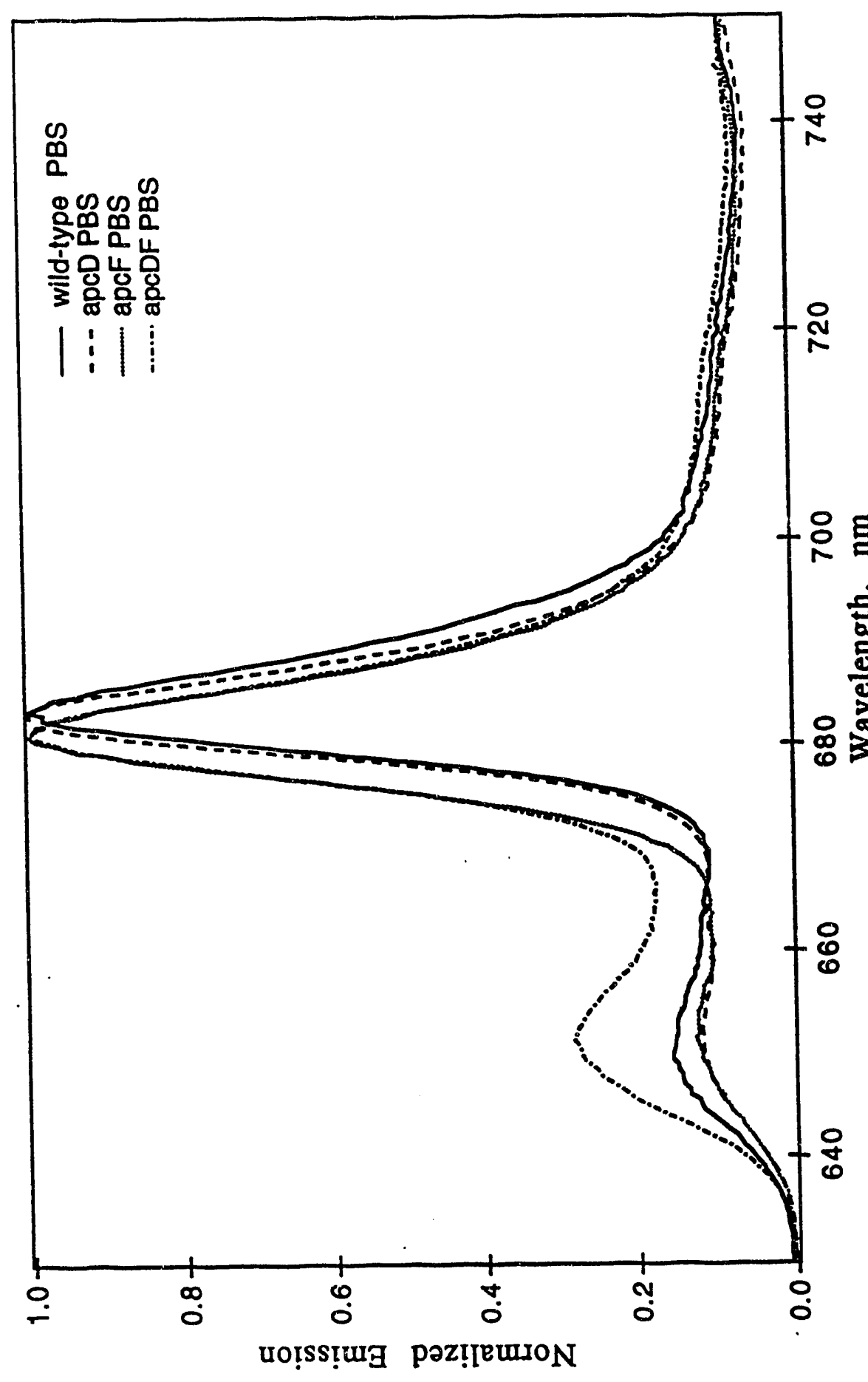

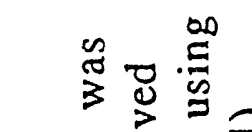

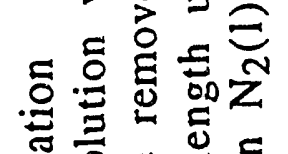

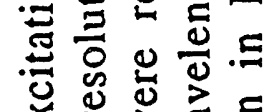

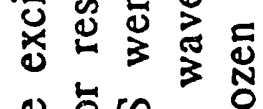

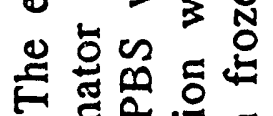

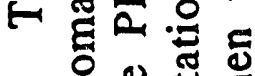

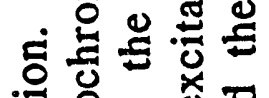

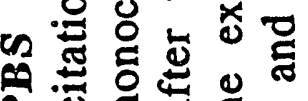

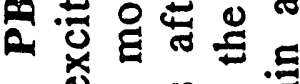

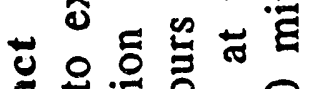

ฐ

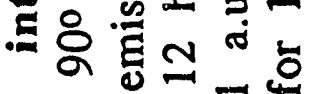

प ত্

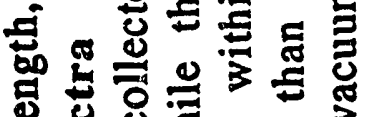

웡 웡

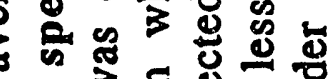

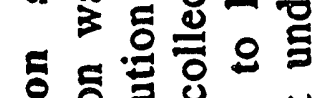

동 응 엉

. \&े

텅 형

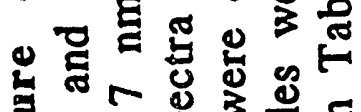

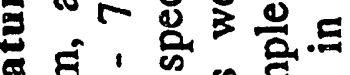

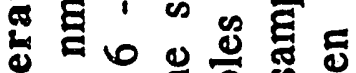

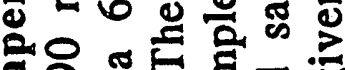

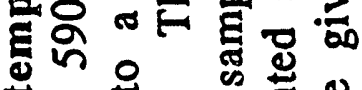

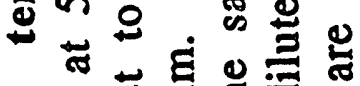

马ुष्ठ

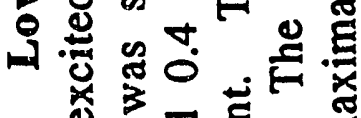

은

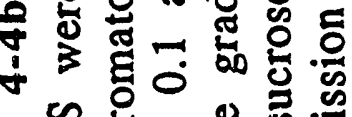

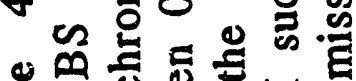

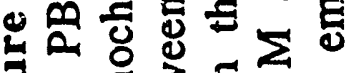

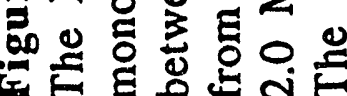


apcDF spectra are indistinguishable from each other within the variability of the experiment. The apcF and apcDF spectra are blueshifted from that of the wild-type by $2 \mathrm{~nm}$.

\section{Time-Resolved Fluorescence Emission}

The kinetics of energy transfer from one pigment pool to another within the PBS complex can be elucidated using timeresolved fluorescence emission. Any major impairment in the energy transfer characteristics of the PBS antenna should be discernible using single photon timing because the measured decay rates at different wavelengths should change.

I begin this section of the chapter by discussing some of the theory behind the energy transfer kinetics in PBS. Holzwarth has written several reviews on the energy transfer in PBS with more details (Holzwarth, 1991; Holzwarth, 1986). The kinetics of energy transfer in the PBS measured by single photon timing indicates that excitation energy within pools of PC or AP very rapidly equilibrates onto the lowest energy chromophores. The transfer of energy within pools of the same pigment occurs on a timescale too fast for us to detect using single photon timing. Energy transfer between pools of dissimilar pigments, such as PC to AP, occurs on a longer timescale such as 25 to 100 ps. This energy transfer step is the rate-limiting step measured using this method of detection.

A model for the energy transfer processes in PBS is given in Figure 4-5 taken from a Holzwarth review (Holzwarth, 1986). I have modified the scheme by adding in boldface print the rates I measured for the wild-type PBS from Synechococcus sp. PCC 7002. 

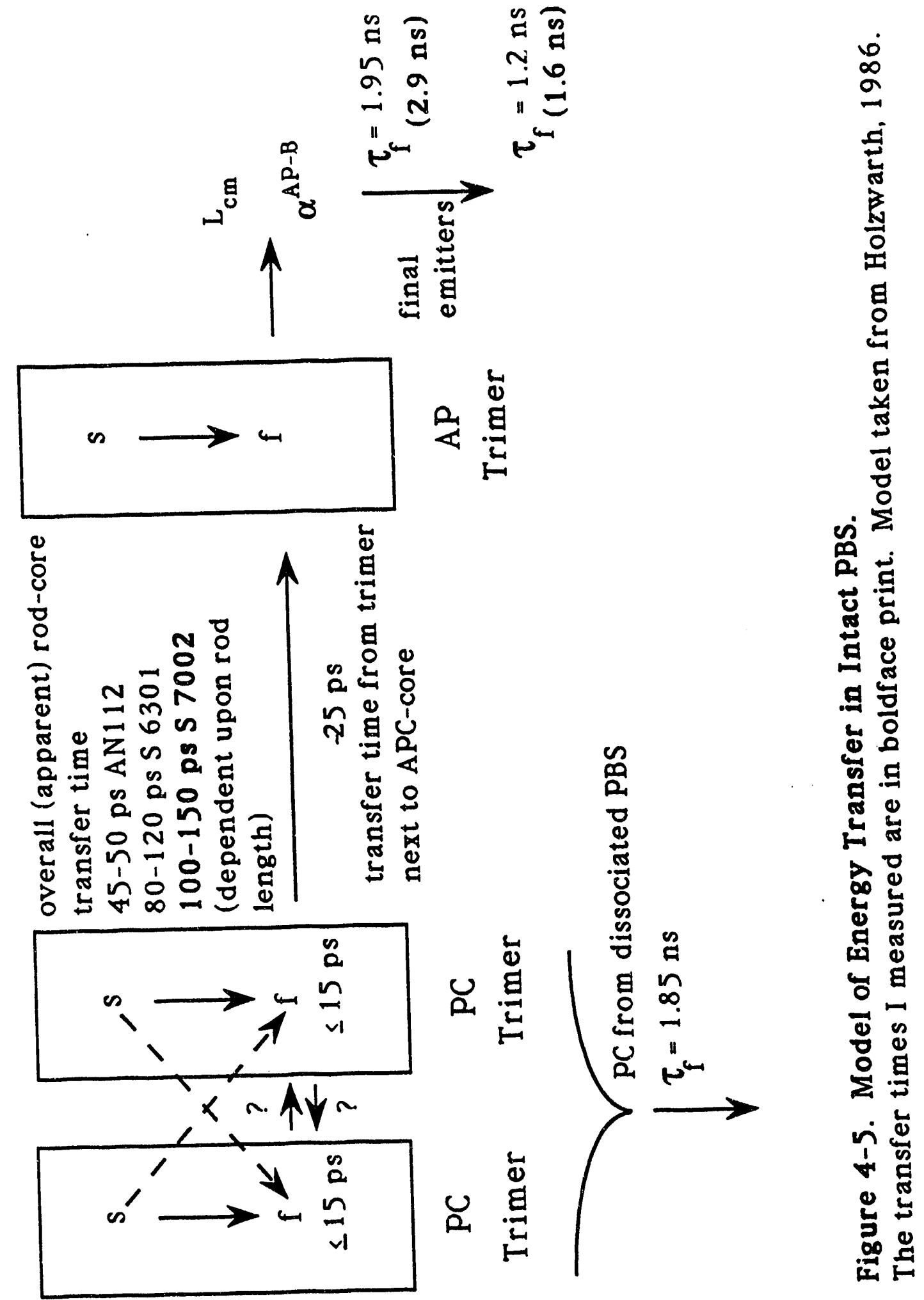
In this model, most of the longer lived emission originates from the terminal emitters. The Boltzmann calculations from earlier in the chapter indicate that as long as the chromophores are coupled for energy transfer, back transfer between the different chromophore pools is significant at RT. Therefore, I think that the two lifetimes attributed to the terminal emitters in Holzwarth's model really apply to all of the coupled chromophores, including PC, $A P$, and the terminal emitters. Because the energy transfer times are much shorter than the decay of the fluorescence, the energy should be equilibrated throughout the PBS complex before much emission occurs.

A competing model has been presented by Glazer, Yeh et al. based upon data obtained from Synechocystis 6701 PBS (Glazer, Yeh et al., 1985; Glazer, Chan et al., 1985). In their model, energy transfer between the chromophores within each subunit is essentially instantaneous with the disk-to-disk transfer step being the rate-limiting transfer process. In this model, they describe the phycobilisome as a lattice of approximately 625 chromophores in which energy transfer can be described as a linear five-point array. The significant differences between the Glazer and Holzwarth models is examined in the Holzwarth reviews.

Time-resolved emission decays were collected for each PBS type, and decay-associated spectra (DAS) were calculated for each set of data, as shown in Figures 4-6a to 6f. As discussed in Chapter 2, each decay was fit to a sum of exponentials using global analysis. At least three components were required for a good fit. The fluorescence decays for different strains were collected using 


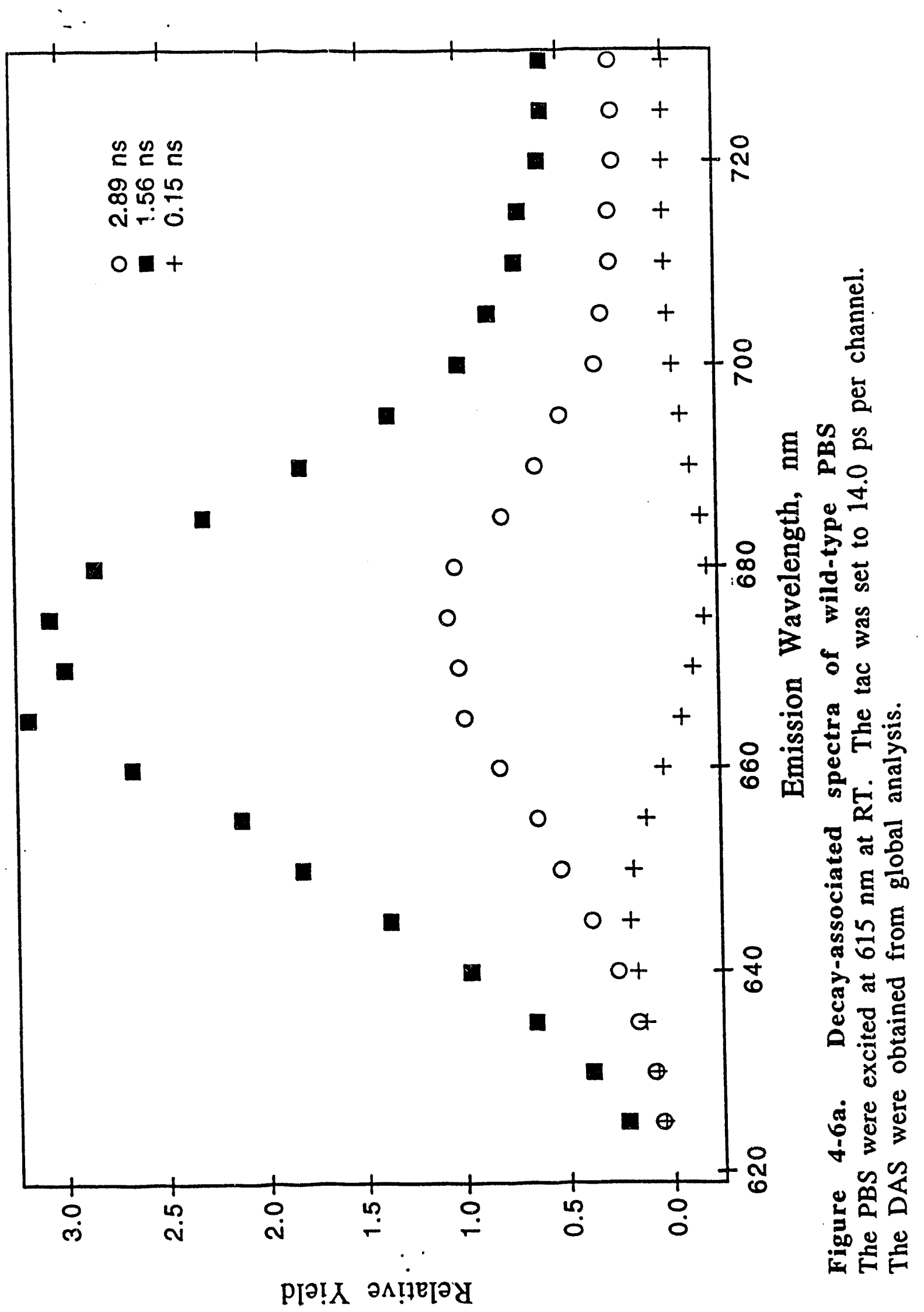




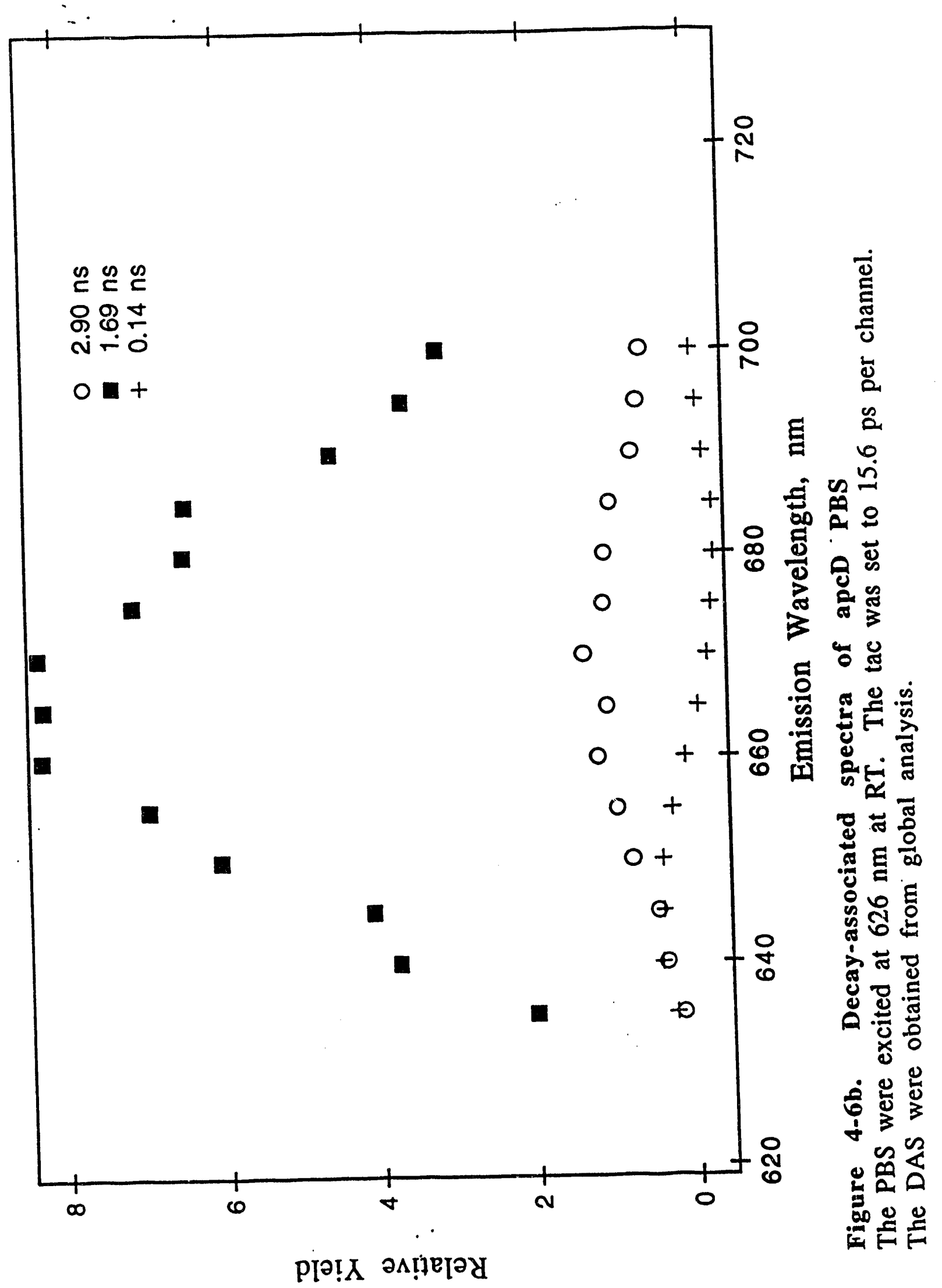


110

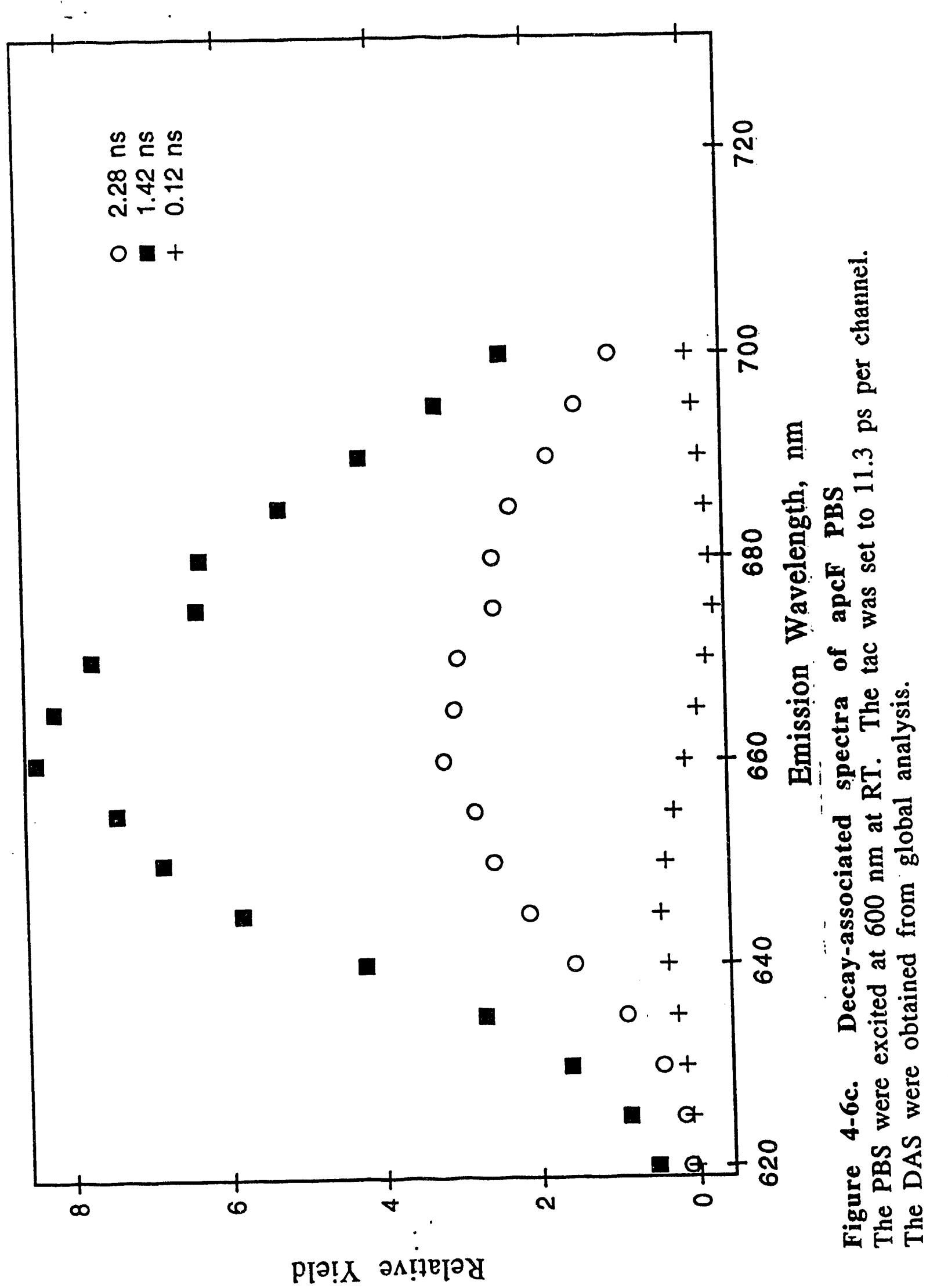


111

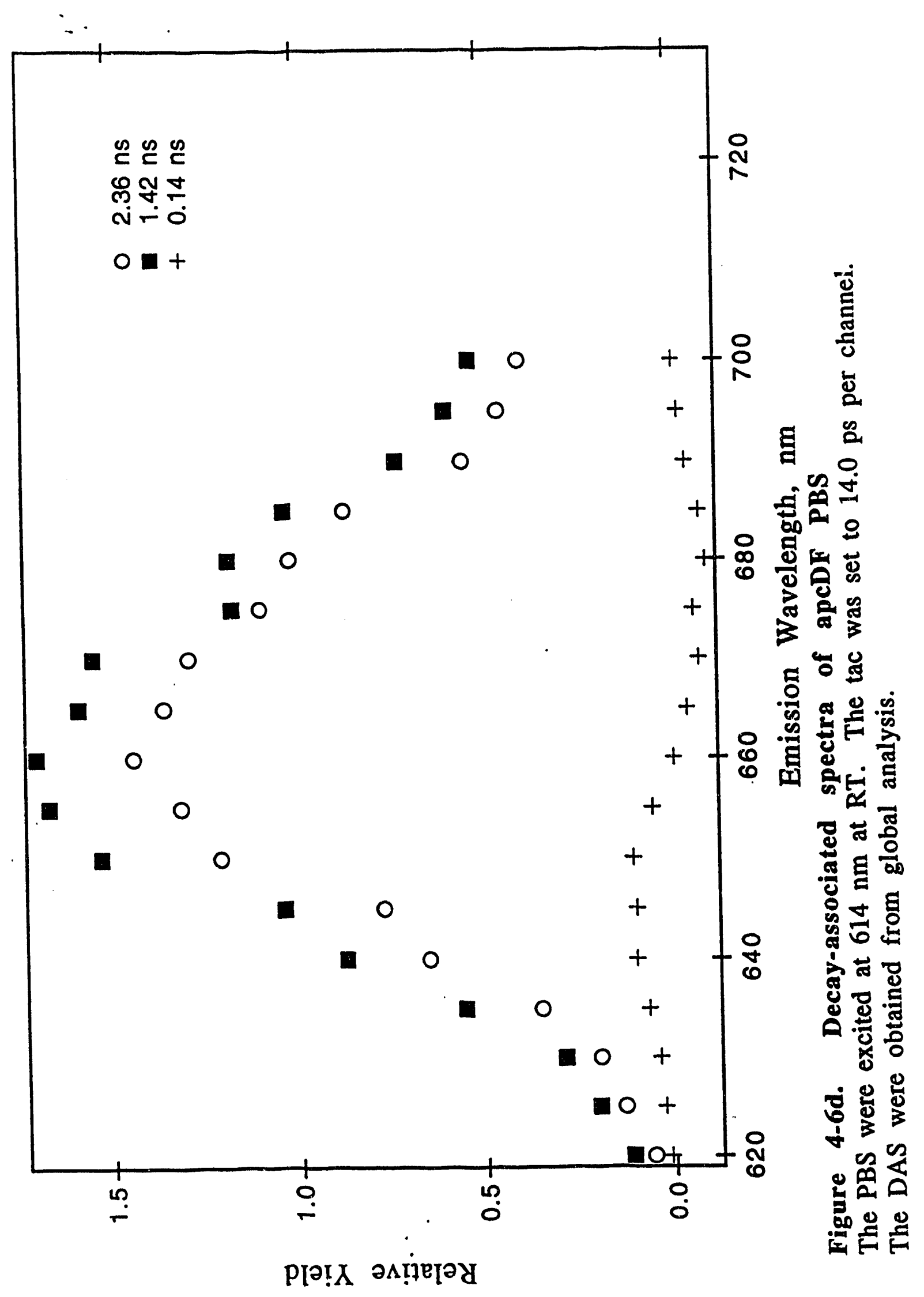




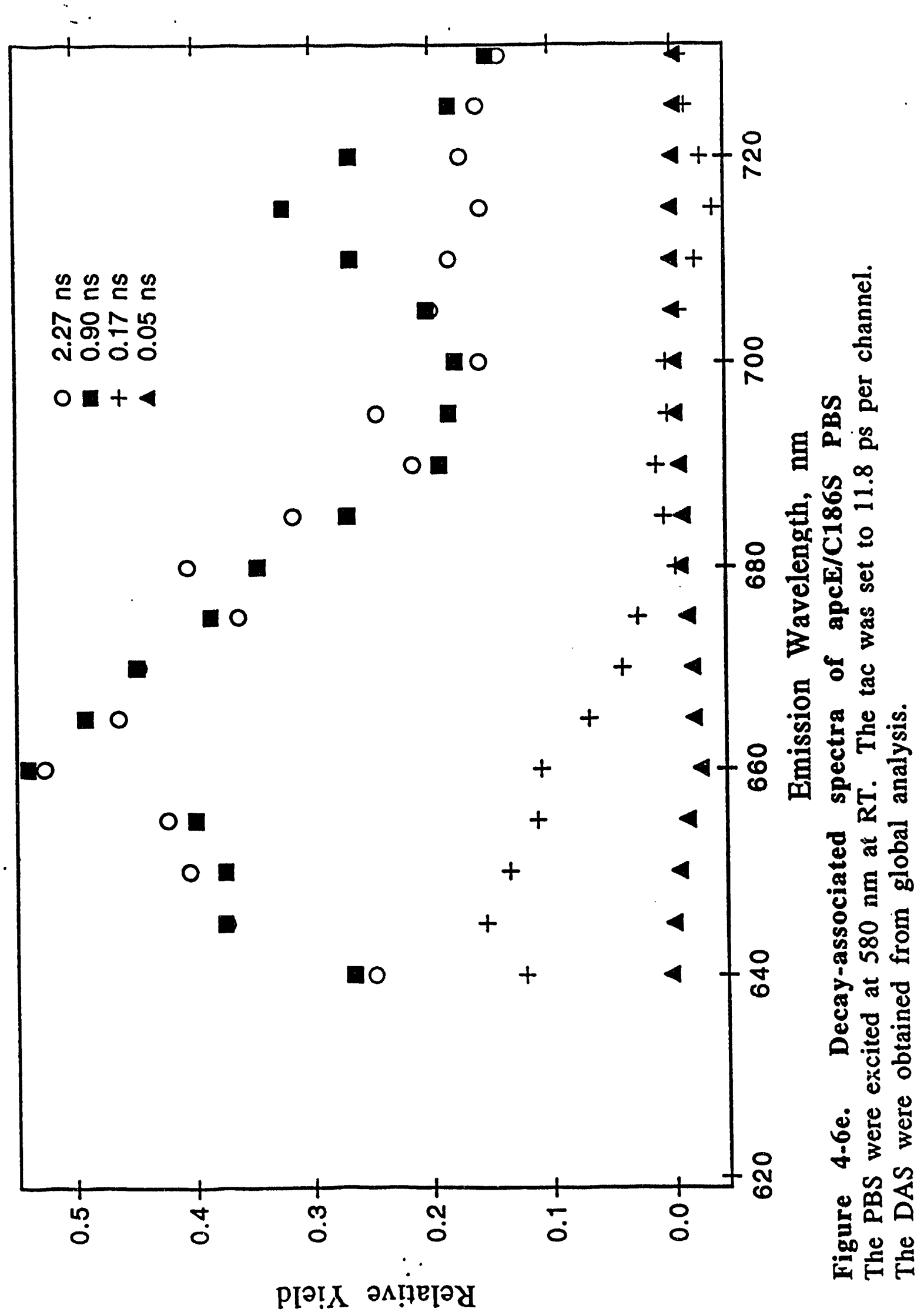


113

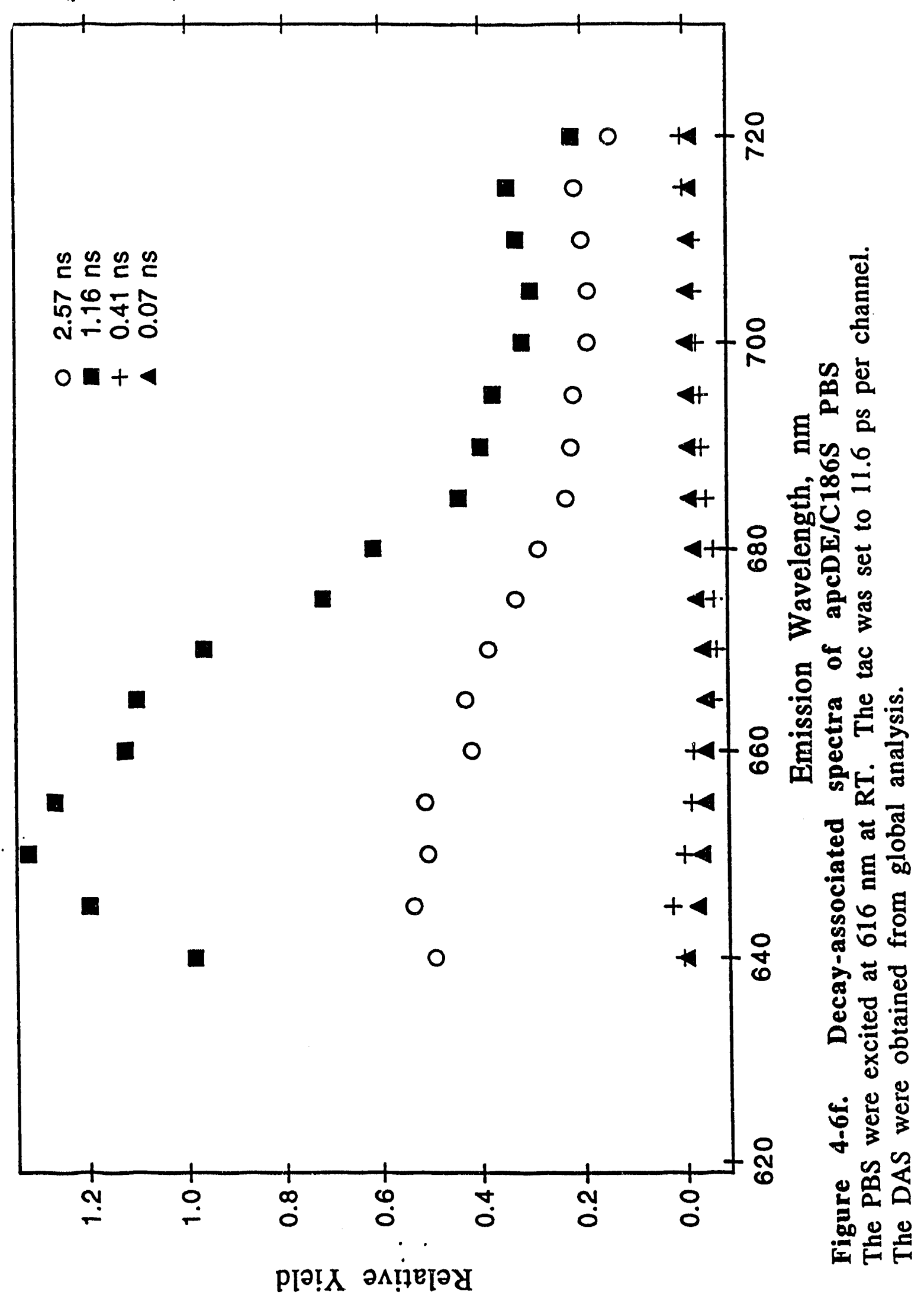


excitation wavelengths ranging from 580 to $625 \mathrm{~nm}$. Within this range of excitation wavelengths, the decay components required to fit the data for each strain did not seem to change, although the relative yield of each component did vary with excitation wavelength. This conclusion is based on examination of wild-type DAS obtained at many different wavelengths.

The time-resolved data that I obtained for the wild-type PBS are similar to those found in the literature for intact PBS from Mastigocladus laminosus (Hefferle, Nies et al., 1983) and Synechococcus 6301 (Gillbro, Sandström et al., 1985; Suter, Mazzola et al., 1984). The data in the literature were obtained for single fits at different excitation and emission wavelengths; no global analysis was applied. From the Gillbro paper, excitation at 581 or $620 \mathrm{~nm}$ with $680 \mathrm{~nm}$ emission gave fluorescence decays which required three components of approximately $0.05 \mathrm{~ns}, 1.4 \mathrm{~ns}$, and $2 \mathrm{~ns}$ for a good fit. For the same excitation and emission wavelengths used in the Gillbro paper, the Suter paper reported three components of $0.13 \mathrm{~ns}, 1.0 \mathrm{~ns}$, and $1.9 \mathrm{~ns}$. The Hefferle study of $M$. laminosus reported lifetimes of around 0.1 and $1.4 \mathrm{~ns}$. This study used streak camera detection which was why they probably couldn't resolve the longest-lived component. Feis, Friedrich et al. also studied energy transfer in PBS from $M$. laminosus using hole-burning techniques; they found an energy transfer time of 147 ps from AP to the terminal emitters (Feis, Friedrich et al., 1992).

As I mentioned earlier in the chapter, a certain amount of emission is expected from the PC and AP chromophores at RT due to the large populations of PC and AP chromophores. Because these 
chromophores are coupled with the terminal emitters, their enission will decay with the same time constant as the terminal emitters after a certain equilibration time (approximately $0.15 \mathrm{~ns}$ is needed for equilibration between the wild-type chromophores). This is why the lifetime components assigned to the terminal emitters span the emission spectrum from 640 to $690 \mathrm{~nm}$, as shown in Figure 4-6a.

The apcD, apcF, apcDF, and wild-type PBS decays all required three components for good fits. The apcD and wild-type DAS, Figures 4-6a,6b, are indistinguishable from each other with each DAS containing three components of approximately $2.9 \mathrm{~ns}, 1.6 \mathrm{~ns}$, and 0.15 ns. Both DAS contain a 0.15 ns component, which has a positive amplitude at about $650 \mathrm{~nm}$ and a negative amplitude at $680 \mathrm{~nm}$. The negative amplitude or "risetime" indicates energy transfer to the $680 \mathrm{~nm}$ emitters from AP. and PC in the PBS. One can think of the risetime as the time required for equilibration of energy between the different chromophore types.

The apcF and apcDF DAS are indistinguishable from each other and only slightly different from that of the wild-type, Figures 4$6 c, 6 \mathrm{~d}$. The two long-lived decay components found in the apcF and apcDF DAS, assigned as the lifetimes of the terminal emitters, are slightly shorter than those of the wild-type. The shortest component, $0.14 \mathrm{~ns}$, is very similar to that of the wild-type. The mutations didn't cause any differences in the equilibration time between the chromophores.

The DAS spectra for the apcDE/C186S and apcE/C186S mutants are aitered from that of the wilu-type; a minimum of four 
components was necessary to obtain good fits for the decays. The DAS obtained for the apcDE/C186S PBS did vary somewhat with preparation. The apcDE/C186S PBS were a little more susceptible to dissociation then the other mutants.

The two longer-lived decay components found in the apcDE/C186S and apcE/C186S DAS of approximately 2.5 and 1 ns are assigned to the terminal emitters: The shortest decay component, approximately $0.06 \mathrm{~ns}$, has no counterpart in the wildtype DAS, and it is of very low yield. The remaining decay component of $0.17 \mathrm{~ns}$ in apcE/C186S DAS and $0.4 \mathrm{~ns}$ in apcDE/C186S DAS is similar to the $0.15 \mathrm{~ns}$ component found in the wild-type DAS.

The modified chromophore found in the apcDE/C186S and apcE/C186S PBS appears to decay with a lifetime of approximately 1 ns. It still is coupled to other chromophores in the PBS as shown by the emission of the $1 \mathrm{~ns}$ component from 640 to $680 \mathrm{~nm}$ and the $0.17 \mathrm{~ns}$ risetime present at $715 \mathrm{~nm}$.

\section{Conclusions}

PBS from the mutants dissociated easier under the conditions for their isolation than the wild-type PBS. The apcE/C186S and apcDE/C186S PBS proved to be the most unstable. This was determined by looking at the amount of free phycobiliprotein present on the sucrose gradient.

The absorption spectra of the apcD and apcF PBS are very similar to that of the wild-type PBS, but the absorption spectra for the apcDF, apcDE/C186S, and apcE/C186S PBS display less 
absorption on the red edge of the spectrum than the wild-type PBS. The difference spectra for each of the mutants confirm that conclusion.

The difference spectra also indicate that PBS from the apcDE/C186S and apcE/C186S mutants absorb less at approximately 675 to $680 \mathrm{~nm}$ (due to the lack of normal $\mathrm{L}_{\mathrm{cm}}$ chromophore) than the wild-type but more at $695 \mathrm{~nm}$ (due to the addition of the modified chromophore). The difference spectra for the other mutants are more inconclusive; the magnitude of the differences are smaller. I will examine the absorption spectra of the core preparations in the next chapter to see if the differences are consistent.

The RT emission spectra for the apcD, apcF, and apcDF PBS were slightly red-shifted from that of the wild-type PBS. The differences could be explained qualitatively by calculating the equilibrated excited state populations using a Boltzmann distribution. When the antenna complex is loses two or four of the six $680 \mathrm{~nm}$ emitters, the equilibrium populations of the excited chromophores change. This results in more steady state emission originating from PC and AP chromophores.

The apcD, apcF, and apcDF emission spectra qualitatively illustrated this result. The apcD and apcF PBS are missing one of the three $680 \mathrm{~nm}$ emitters, and their emission spectra are blueshifted about $10 \mathrm{~nm}$ from that of the wild-type. The apcDF PBS is missing two of the three $680 \mathrm{~nm}$ emitters, and it's emission spectrum is blue-shifted by about $15 \mathrm{~nm}$ from that of the wildtype. The changes in emission spectra of the PBS from the apcD, 
apcF, and apcDF strains appear to be described by the Boltzmann calculations.

The Boltzmann calculations did not work well for the apcDE/C186S and apcE/C186S mutants. The fluorescence yield from the modified chromophore is low; it was found to be only $30 \%$ instead of the calculated $75 \%$ of the total yield. The Boltzmann predictions could break down for the modified chromophore because the chromophore undergoes more nonradiative deactivation. The modified chromophore acts to quench the emission from the PBS antenna such that the quantum yield of fluorescence is only $50 \%$ of the wild-type.

Also, the Boltzmann calculations predict only small amounts of $680 \mathrm{~nm}$ emission should be present in the apcE/C186S and apcDE/C186S spectra assuming all the chromophores are coupled. No $680 \mathrm{~nm}$ emission band could be resolved in the apcDE/C186S or apcE/C186S spectra. This indicates that the $680 \mathrm{~nm}$ emitters present are coupled to the $715 \mathrm{~nm}$ emitter; the $\alpha$ AP-B and $\beta^{18}$ chromophores transfer energy to the modified chromophore. This result is contrary to what Mimuro, Yamazaki et al. found. They reported that at least two of the terminal emitters are independent of each other (Mimuro, Yamazaki et al., 1989).

At $77 \mathrm{~K}$, the back transfer of energy from the $680 \mathrm{~nm}$ emitters to the other chromophores is much less important. Only the terminal emitters should fluorescence at $77 \mathrm{~K}$ if all the chromophores are coupled. This is the case in the wild-type, apcD, apcF, and apcDF emission spectra. The majority of the emission appears to be from one chromophore type. 
The low temperature emission spectra from the apcDE/C186S and apcE/C186S PBS are much more complicated; more than one emission band is present. At $77 \mathrm{~K}$, emission is seen from the 680 $\mathrm{nm}$ emitters and from the modified chromophore along with a third emission band from dissociated PC. It is possible that the PBS from these mutants undergo structural rearrangement when frozen, but I favor another explanation. The spectral overlap between the absorption spectrum of the $680 \mathrm{~nm}$ emitter and the emission spectrum of the $715 \mathrm{~nm}$ emitters decreases at $77 \mathrm{~K}$ because both the absorption and emission spectra narrow with lowering temperature. Because the overlap integral decreases, energy transfer between the two chromophore type is less favorable, and more emission is seen from the $680 \mathrm{~nm}$ emitter.

From the low temperature data, I assign emission maxima to the different chromophores. The $\beta 18$ chromophore emits at $678 \mathrm{~nm}$ in the absence of the $\mathrm{L}_{\mathrm{cm}}$ chromophore, as shown by the apcDE/C186S spectrum. The $\alpha$ AP-B chromophore emits around $682 \mathrm{~nm}$, as shown by the apcE/C186S spectrum. Therefore, from the wild-type spectrum, the wild-type $\mathrm{L}_{\mathrm{cm}}$ chromophore must emit at $683 \mathrm{~nm}$.

The wild-type and apcD emission spectra are almost indistinguishable from each other, as are the apcF and apcDF spectra. I can explain the differences in the $a p c D$, apcF, and apcDF spectra by attributing the emission at low-temperature to mainly the $\mathrm{L}_{\mathrm{cm}}$ chromophore. As shown in the wild-type emission spectrum, the $\mathrm{L}_{\mathrm{cm}}$ chromophore emits at $683 \mathrm{~nm}$ when the PBS core is intact with all the polypeptides present. As I will show in the next chapter, the emission spectrum of $\mathrm{L}_{\mathrm{cm}}$ blue-shifts in the 
absence of the $\beta 18$ subunit. Therefore, the low-temperature emission spectra of the apcF and apcDF PBS are slightly blue-shifted from that of the wild-type because the $\beta^{18}$ subunit is missing.

The $\alpha$ AP-B chromophore emits at a higher energy than the $L_{c m}$ chromophore, so the $\alpha$ AP-B chromophore must transfer its energy to the $\mathrm{L}_{\mathrm{cm}}$ chromophore at $77 \mathrm{~K}$. Bryant has suggested that the $\alpha \mathrm{AP}-\mathrm{B}$ subunit is active in transferring energy to the PSI reaction center (Bryant, personal communication). If this hypothesis is correct, then the $\alpha$ AP-B chromophore might only play a role in energy transfer in the PBS under high light conditions.

In the time-resolved fluorescence data, the apcD and wild-type PBS were indistinguishable from each other, as were the apcDF and apcF PBS. The results from the apcF and apcDF PBS were only slightly changed from that of the wild-type with the longest lived decay components shortened slightly. This could possibly be due to a small degree of structural disorganization in the PBS core of the apcF and apcDF PBS.

The time-resolved data obtained for the apcDE/C186S and apcE/C186S mutants is altered from that of the wild-type. The longest lived components were somewhat shorter than those seen in the wild-type DAS, while the shortest component resolved in the apcDE/C186S and apcE/C186S DAS has no counterpart in the wildtype DAS. This component possibly indicates impaired energy transfer from the rods to the core. The process is slowed enough that it is now detectable using single photon timing.

From the time-resolved emission work, I assign a lifetime of approximately $1 \mathrm{~ns}$ to the modified chromophore and show that the 
modified chromophore is coupled to the other chromoproteins present, as shown by the presence of a risetime at $715 \mathrm{~nm}$. There is other evidence of $\mathrm{AP}$ and $\mathrm{PC}$ coupling to the modified chromophore; AP and PC emission decays with the same 1 nls lifetime.

In the next chapter I will examine the spectral characteristics of a subcore preparation which is missing the $\alpha$ AP-B chromophore. I will also discuss the evidence of an interaction between the $L_{c m}$ and $\beta 18$ chromophores. 


\section{References for Chapter 4}

de Lorimier, R. M., R. L. Smith and S. E. Stevens, Jr. (1992). Plant Physiol. 98: 1003-1010.

Feis, A., J. Friedrich, L. Gottschalk and H. Scheer (1992). J. Phys. Chem. 96: 6087-6089.

Gillbro, T., A. Sandström, V. Sundström, J. Wendler and A. R. Holzwarth (1985). Biochim. Biophys. Acta 808: 52-65.

Glazer, A. N., and D. A. Bryant (1975). Arch. Microbiol. 104:15-22.

Glazer, A. N., S. W. Yeh, S. P. Webb, and J. H. Clark (1985). Science 227:419-423.

Glazer, A. N., C. Chan, R. C. Williams, S. W. Yeh, and J. H. Clark (1985). Science 230:1051-1053.

Hefferle, P., M. Nies, W. Wehrmeyer and S. Schneider (1983). Photochem. Photobiol. 5: 41-51.

Holzwarth, A. R. (1986). Photochem. Photobiol. 43: 707-725.

Holzwarth, A. R. (1991). Physiol. Plant. 83: 518-528.

Lundell, D. J., G. Yamanaka and A. N. Glazer (1981). J. Cell. Biol. 91: 315-319.

Mimuro, M., I. Yamazaki, N. Tamai and T. Katoh (1989). Biochim. Biophys. Acta 973: 153-162.

Suter, G. W., P. Mazzola, J. Wendler and A. R. Holzwarth (1984). Biochim. Biophys. Acta 766: 269-276. 
Chapter 5. Discussion of 18 S Preparation Results

In the last chapter I examined the absorption and emission characteristics of the intact PBS antenna for wild-type and mutant strains. In this chapter I will discuss the spectral properties of a subcore preparation, the $18 \mathrm{~S}$ preparation, in which only a fraction of the core is present with a small amount of PC.

According to the paper by Gingrich, Lundell, and Glazer in which they isolate an $18 \mathrm{~S}$ preparation from Synechocystis 6701, a cyanobacterium containing PBS with tricylindrical cores, the relative ratios of $\alpha^{\mathrm{AP}}: \beta \mathrm{AP}: \alpha^{\mathrm{PC}}: \beta^{\mathrm{PC}}: \mathrm{L}_{\mathrm{cm}}$ is approximately 6: 6: 3: 3: 1 (Gingrich, Lundell, et al., 1983). No $\alpha$ AP-B appeared to be present in their preparation. Also, even though they didn't explicitly say that $\beta^{18}$ is present, based upon work done with Synechococcus $6301, I$ am assuming that the $\beta 18$ subunit is present in the same concentration as the $\mathrm{L}_{\mathrm{cm}}$ (Yamanaka, Lundell, et al., 1982; Lundell and Glazer, 1983). This assumption should be valid because the $L_{c m}$ and $\beta^{18}$ polypeptides are in the same trimer.

I decided to use this preparation to study the spectral properties of the $\beta^{18}$ and $\mathrm{L}_{\mathrm{cm}}$ chromophores because the emission spectrum for the subcore preparation was found to be very similar to that of intact PBS (Sandström, Gillbro, et al., 1988).

I ran samples of wild-type and apcF $18 \mathrm{~S}$ preparations on a HPLC column using the procedure developed by Swanson and Glazer (Swanson and Glazer, 1990). I was able to confirm the presence of both AP subunits, both PC subunits, the $\mathrm{L}_{\mathrm{cm}}$ polypeptide, and two colorless polypeptides of approximately $30 \mathrm{kD}$. The wild-type 
preparation also contains an additional chromopeptide present at approximately the same concentration as the $\mathrm{L}_{\mathrm{cm}}$ polypeptide. I found this protein to be approximately $18 \mathrm{kD}$ in molecular weight using SDS-PAGE, and I identified it to be the $\beta^{18}$ subunit. The apcF preparation was missing that polypeptide.

I did not find any polypeptide, corresponding to the $\alpha$ AP-B subunit to be present in the18 $\mathrm{S}$ preparations I ran on the HPLC. I was not able to get consistent data on the relative amounts of $\beta \mathrm{AP}$ and $\alpha$ AP present in the preparations. From the HPLC data I couldn't determine if a $\beta$ AP subunit is substituting for the missing $\beta^{18}$ subunit in the apcF preparation.

The modified chromophore present in the apcDE/C186S and apcE/C186S cells and intact PBS is missing in the $18 \mathrm{~S}$ preparation. Therefore, the $18 \mathrm{~S}$ preparation should be composed only of PC, AP, and the $\beta 18$ chromophores for the apcDE/C186S and apcE/C186S preps and only PC, AP, and $\mathrm{L}_{\mathrm{cm}}$ chromophores for the apcDF and apcF preps. The absence of the modified chromophore is not unexpected since it was thought to be only noncovalently associated with the PBS antenna.

I successfully prepared $18 \mathrm{~S}$ preparations for all of the mutants, but the yield of the preparation from the apcDE/C186S cells was consistently low. It also was contaminated with PC. I did not carry out any time-resolved fluorescence studies on the apcDE/C186S mutant because of the lack of sufficient sample, but I do have steady state absorption and emission data for the apcDE/C186S preparation. 
I would like to emphasize one more point before discussing the data. Since the $\alpha$ AP-B is absent from the preparation, I expect the compositions of the apcD and wild-type preparations to be identical. The same should hold true for the apcF and apcDF preparations and the apcDE/C186S and apcE/C186S preparations. You will notice that spectral characteristics of the preparations are not always consistent. I concentrated my energies in preparing good samples of wild-type, apcF, and apcE/C186S preparations as judged by the $77 \mathrm{~K}$ emission spectra, and the quality of spectral data from the other three mutants is questionable at times.

\section{Absorption}

As I did in Chapter 4 with intact PBS, I make the assumption that the absorption of the chromophores in the $18 \mathrm{~S}$ preparation is additive. The absence of a chromophore will leave a hole in the absorption spectrum of the preparation. The absorption spectra of the $18 \mathrm{~S}$ core preparations are shown in Figure 5-1a,1b. I found some variability in the absorption spectra of my $18 \mathrm{~S}$ preparations. The absorption maxima for different preparations of the same strain differed by as much as $2 \mathrm{~nm}$. The spectra displayed in Figures 5-1a,1b are an average of spectra from different preparations weighted equally. I averaged the spectra to get a more meaningful difference spectra as I will discuss later.

All of the preparations have absorption maxima around $653 \mathrm{~nm}$, with the exact position given in Table 5-1. As shown by the location of the absorption maximum, I was successful in isolating a PBS fraction that contains mostly AP. The preparation isolated from 


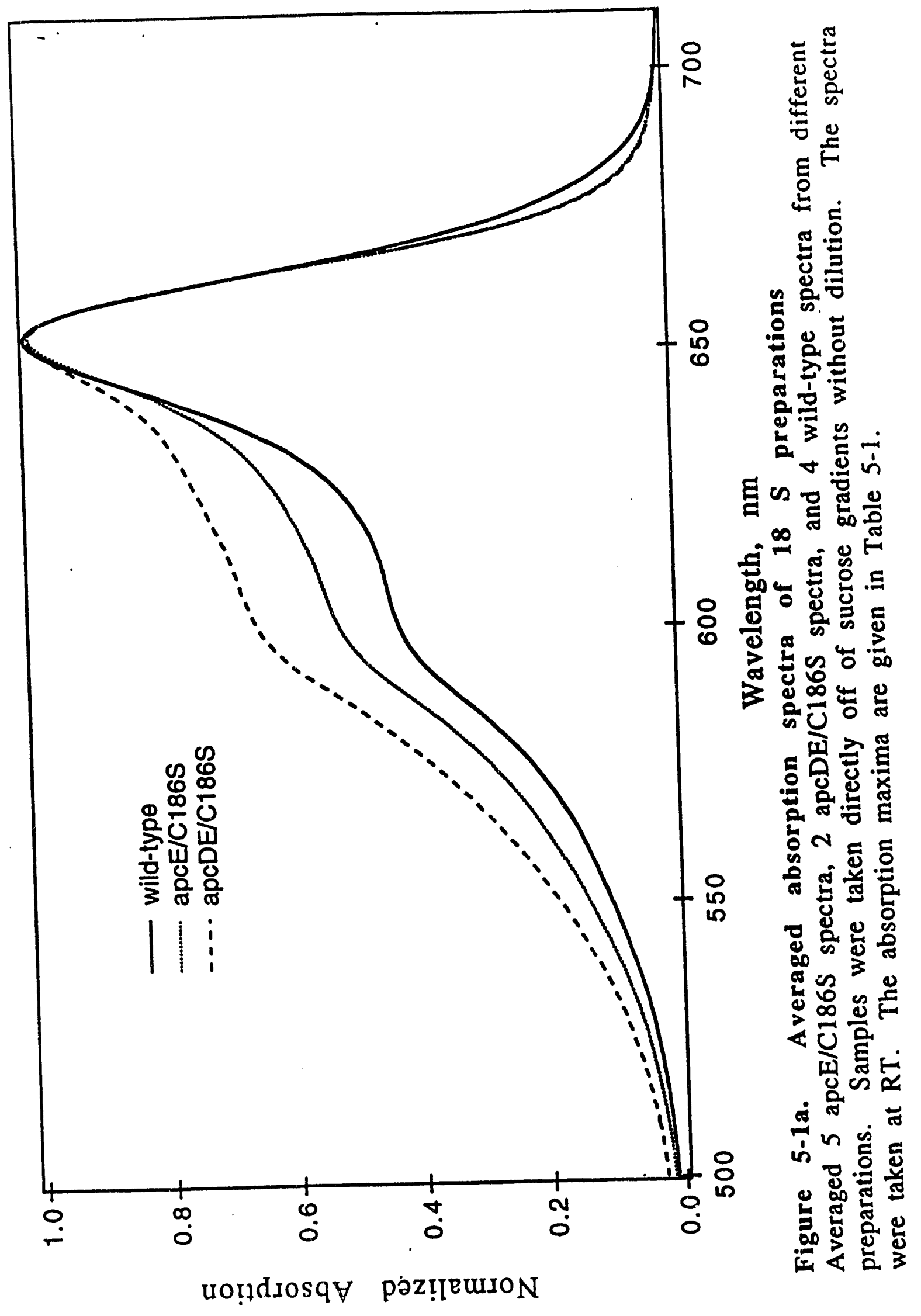




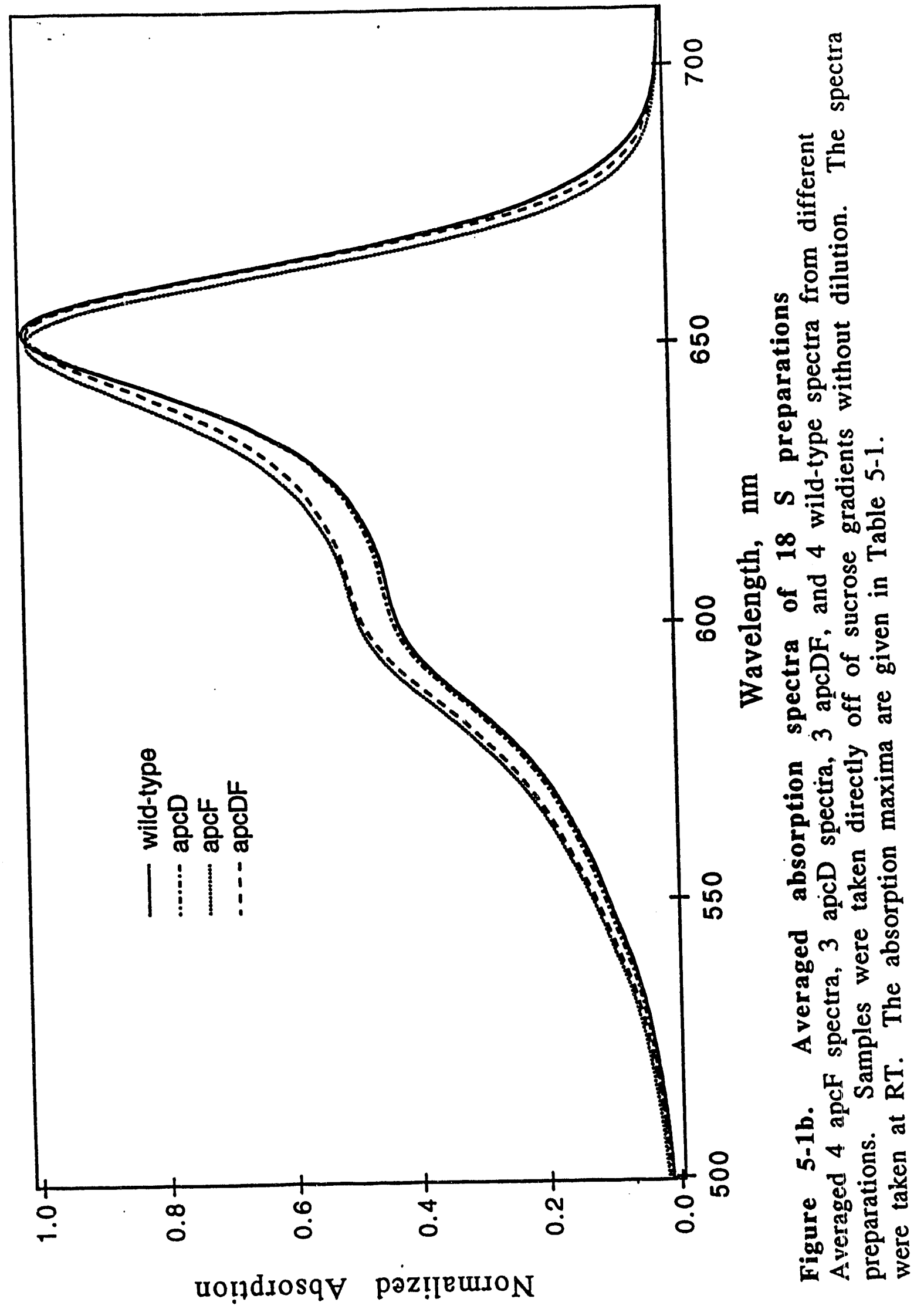


Table 5-1

18 S Preparation Spectral Data

\begin{tabular}{|c|c|c|c|}
\hline & $\begin{array}{l}\text { Absorption } \\
\text { Maximum }\end{array}$ & $\begin{array}{c}\text { Room } \\
\text { Temperature } \\
\text { Emission } \\
\text { Maximum }\end{array}$ & $\begin{array}{c}\text { Low } \\
\text { Temperature } \\
\text { Emission } \\
\text { Maximum }\end{array}$ \\
\hline wild-type & $653 \mathrm{~nm}$ & $668 \mathrm{~nm}$ & $\begin{array}{c}682.5 \mathrm{~nm} \\
653 \mathrm{~nm}\end{array}$ \\
\hline apcD & $653 \mathrm{~nm}$ & $668 \mathrm{~nm}$ & $\begin{array}{c}681.5 \mathrm{~nm} \\
653 \mathrm{~nm}\end{array}$ \\
\hline apcF & $651 \mathrm{~nm}$ & $662 \mathrm{~nm}$ & $\begin{array}{c}676 \mathrm{~nm} \\
651.5 \mathrm{~nm}\end{array}$ \\
\hline apcDF & $652 \mathrm{~nm}$ & $666 \mathrm{~nm}$ & $\begin{array}{c}672.5 \mathrm{~nm} \\
653 \mathrm{~nm}\end{array}$ \\
\hline apcE/C186S & $653 \mathrm{~nm}$ & $666 \mathrm{~nm}$ & $\begin{array}{ll}671 & \mathrm{~nm} \\
646 & \mathrm{~nm}\end{array}$ \\
\hline apcDE/C186S & $653 \mathrm{~nm}$ & $665 \mathrm{~nm}$ & $\begin{array}{ll}670.5 & \mathrm{~nm} \\
647.5 \mathrm{~nm}\end{array}$ \\
\hline
\end{tabular}


Synechocystis 5701 was reported to contain a 2: 1 ratio of AP to PC (Gingrich, Lundell, et al., 1983). The shoulder at approximately 600 $\mathrm{nm}$ is due to PC absorption with a small contribution from AP. The relative height of the shoulder reflects the amount of PC present in the sample. This point is especially well illustrated in Figure 5-1a; the apcDE/C186S spectrum shows a much larger absorption shoulder at $000 \mathrm{~nm}$ due to large amounts of accompanying PC.

The important part of the absorption spectrum in this study is the region from about 655 to $700 \mathrm{~nm}$, the red-edge of the spectrum. As shown in Figure 5-1a, the red-edge of the apcE/C186S and apcDE/C186S absorption bands are indistinguishable from each other. Both show an absorption decrease at about $670 \mathrm{~nm}$ compared to the wild-type preparation.

The spectra for the apcD, apcF, and apcDF preparations are shown in Figure 5-1b. The apcDF and apcF absorption spectra are very similar to each other, and the apcD and wild-type spectra are indistinguishable from one another. I found the apcF and apcDF absorption maximum to be consistently blue-shifted from that of all the other strains, as shown in Table 5-1. The blue shift could be partially due to increased PC content, but it also could be due to a structural change in the PBS core. Only the apcF and apcDF preparations are missing an entire polypeptide. The apcDE/C186S and apcE/C186S preparations are missing a chromophore, but the polypeptide is still there. Therefore, I expect to see a larger effect on the absorption spectrum in the apcF and apcDF preparations. The assumption about the additivity of the chromophores in the apcF and apcDF preparations may no longer hold. 
As with the PBS absorption spectra from Chapter 4, I have calculated difference spectra for the preparations from the mutants. The difference spectra are calculated from an average absorption spectrum obtained for each preparation. I normalized each spectrum to an equal area and then subtracted the spectrum of the mutant from that of the wild-type preparation. Once again, I make the assumption that the preparations contain equal numbers of chromophores. This assumption breaks down when PC contamination is present, as in the apcDE/C186S preparation. I also calculated second derivatives of the difference spectra to show the location of the peaks in the spectra.

In Figure 5-2a, I have displayed the difference spectrum for the apcDE/C186S and apcE/C186S preparations. The difference spectra are hard to interpret because the preparations contain different amounts of PC. I made the assumption that the number of chromophores was constant to calculate the difference spectra, but the apcDE/C186S preparation contains more $\mathrm{PC}$ relative to $\mathrm{AP}$ than the wild-type. Therefore, in the apcDE/C186S difference spectrum, PC contamination will show up as negative peaks at 580 and 630 $\mathrm{nm}$ (PC absorption) along with a positive peak at $655 \mathrm{~nm}$ (AP absorption). Except for the apcD mutant, all of the mutants show signs of increased PC content.

Taking the distortion due to differing amounts of $\mathrm{PC}$ present into consideration, the $1 \mathrm{pcE} / \mathrm{C} 186 \mathrm{~S}$ and apcDE/C186S difference spectra are indistinguishable. This result is also shown by the appearance of the second derivative of the spectrum. Both spectra show that 


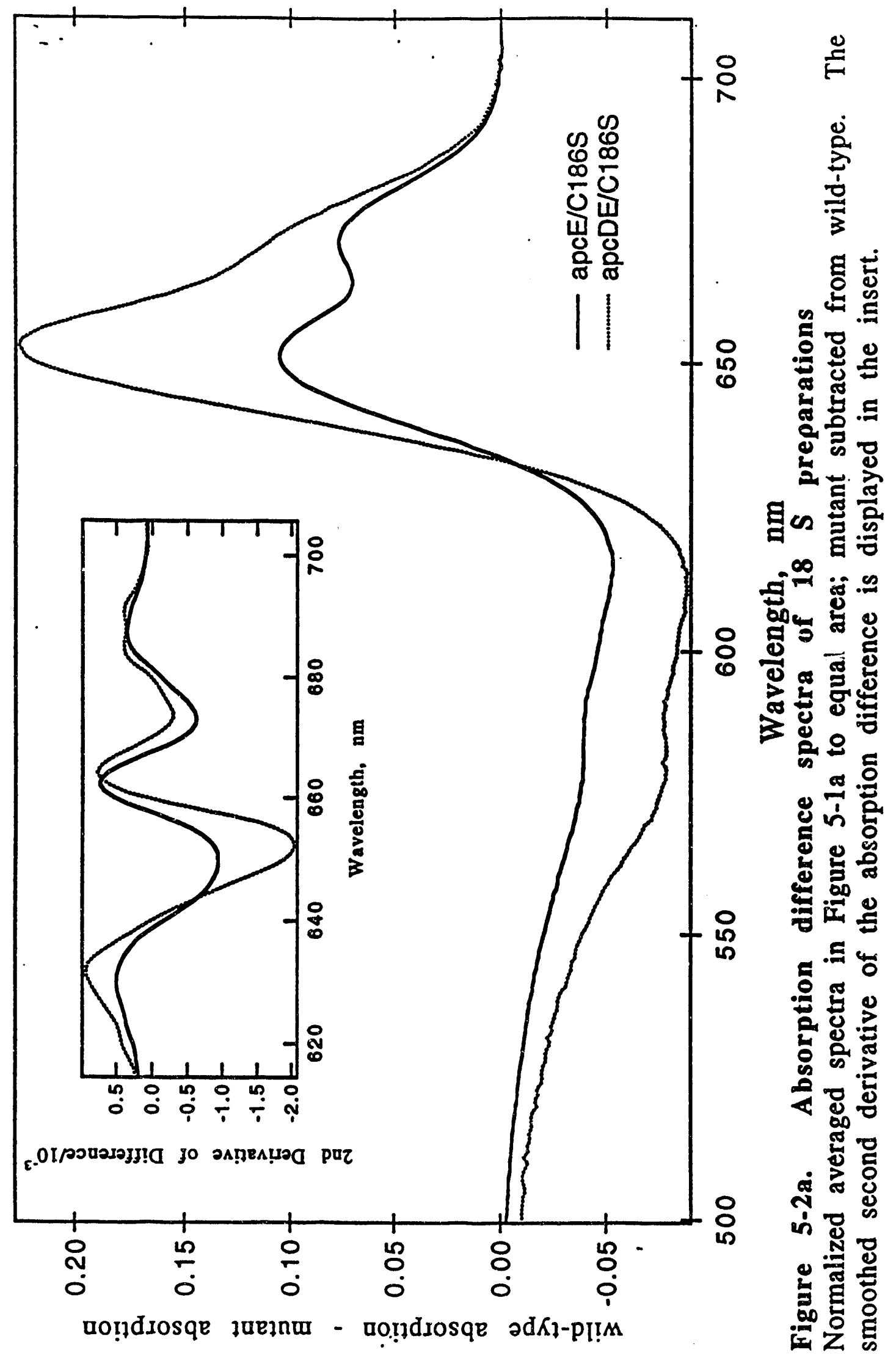




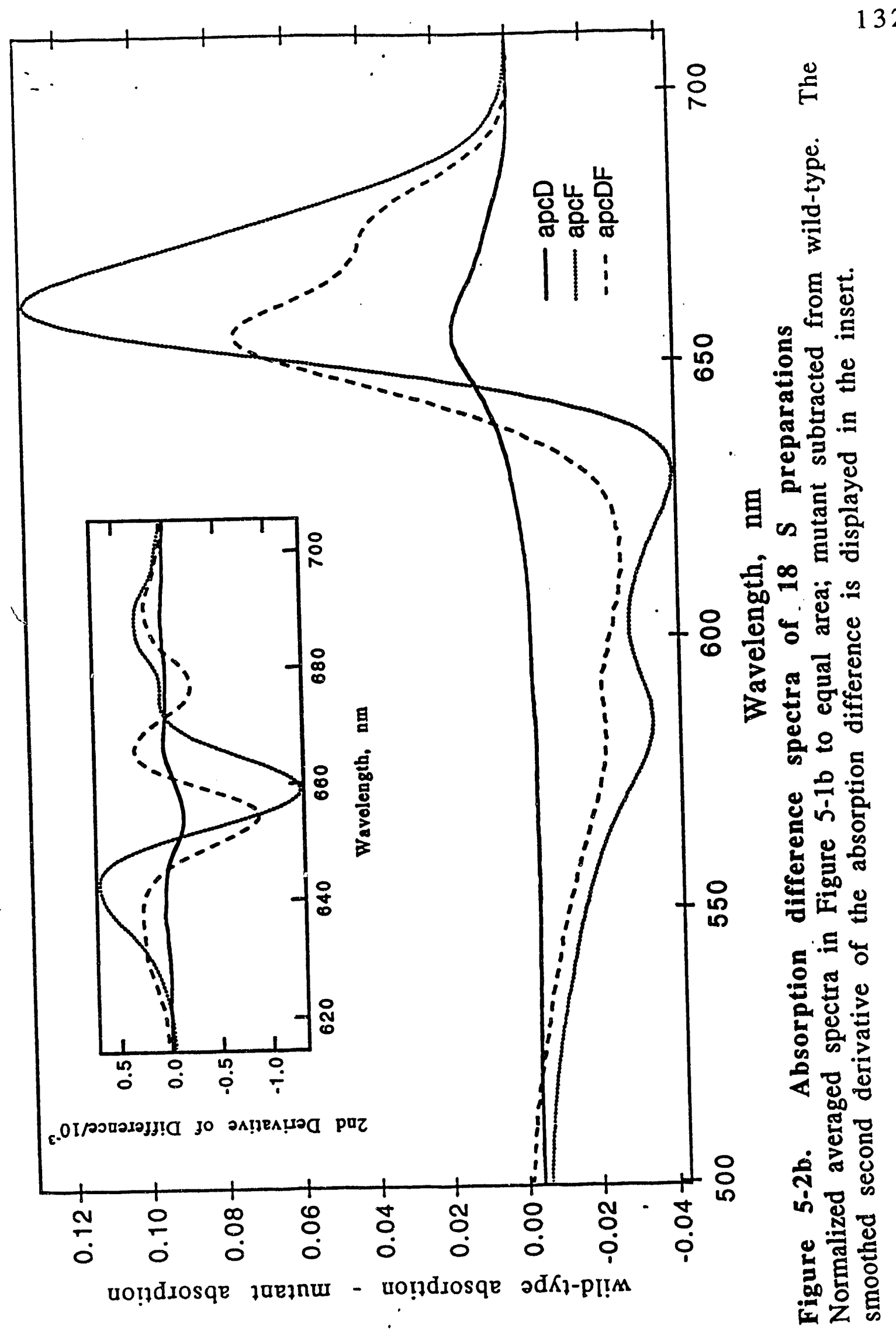


the preparations from the mutants absorb less at $671 \mathrm{~nm}$ than the wild-type.

The difference spectra calculated for the apcD, apcF, and apcDF preparations are shown in Figure 5-2b along with the corresponding second derivatives. The apcD absorption spectrum is indistinguishable from that of the wild-type, as illustrated by the difference spectrum. The apcF and apcDF spectra are more similar to those obtained from the apcDE/C186S and apcE/C186S preparations. As shown earlier in the apcDE/C186S spectrum, the apcF difference spectrum also shows the presence of an increased PC to AP ratio relative to that of the wild-type, with two negative peaks at 580 and $630 \mathrm{~nm}$. The apcF difference spectrum has a large positive peak at approximately $660 \mathrm{~nm}$, which is at least partially due to the increased PC to AP ratio.

The difference spectrum obtained for the apcDF preparation looks very similar to those obtained for the apcDE/C186S and apcE/C186S preparations, except that the shoulder on the $650 \mathrm{~nm}$ peak is closer to $680 \mathrm{~nm}$ rather than $670 \mathrm{~nm}$ as easily seen in the second derivative of the difference. There is some similarity between the second derivatives of the apcF and apcDF spectra. Both second derivatives show the presence of a shoulder at about 680 $\mathrm{nm}$.

\section{Room Temperature Emission}

As discussed in Chapter 4, the room temperature steady state emission spectrum is a reflection of not only the types of chromophores present within the PBS but also of the number of 
each type present. If the chromophores are coupled, back transfer is important at room temperature such that significant amounts of emission will originate from PC and AP in the PBS. This is especially true because larger numbers of PC and AP chromophores are present relative to the $680 \mathrm{~nm}$ emitters in the PBS. The same should hold true for the $18 \mathrm{~S}$ preparations.

I made some Boltzmann calculations as in Chapter 4 using the ratios of AP: PC: $680 \mathrm{~nm}$ emitter as $8: 4.5: 1$ for the wild-type preparation along with the assumption that the $\beta^{18}$ and $L_{c m}$ chromophores emit at $680 \mathrm{~nm}$. I decided on these ratios of chromophores because it seemed likely that the $18 \mathrm{~S}$ preparations consist of the 6 inner AP trimers of the PBS core along with about 3 PC trimers from the rods. I did not find any signs of that the capping $\mathrm{L}^{8}$ is present in the $18 \mathrm{~S}$ preparation from the HPLC data. This would indicate that my core preparation is missing the AP trimers on the ends of the core cylinders. I also assumed that all AP and PC chromophores are equal in energy, and I did not allow for the small differences in the spectral properties of chromophores within each pool.

The Boltzmann calculations show that approximately $60 \%$ of the emission from the wild-type $18 \mathrm{~S}$ preparations should originate from the $680 \mathrm{~nm}$ emitters with most of the rest coming from AP. Only $2 \%$ of the emission should originate from PC chromophores if all of the chromophores are coupled for energy transfer. There is a larger PC contribution than expected in the wild-type spectrum, probably from dissociated PC hexamers contaminating the 
preparation, but the ratio of AP to $680 \mathrm{~nm}$ emission is approximately right.

The emission spectra of the preparations from the mutant and the wild-type strains are shown in Figure 5-3a,3b. In Figure 5-3a, the apcDE/C186S and apcE/C186S emission spectra are almost indistinguishable except that the apcDE/C186S spectrum shows the presence of greater amounts of emission free PC. Boltzmann calculations for the apcDE/C186S and apcE/C186S complexes indicate that $42 \%$ of the emission should originate from the remaining $680 \mathrm{~nm}$ emitter (the $\beta^{18}$ subunit) with $55 \%$ from AP. The amount of emission originating from the $680 \mathrm{~nm}$ emitter in the apcDE/C186S and apcE/C186S spectra appears to be less than $25 \%$, which probably indicates that the $\beta^{18}$ subunit is emitting at a higher energy than $680 \mathrm{~nm}$.

In Figure 5-3b I have displayed the room temperature emission spectra obtained for the apcD, apcF, and apcDF $18 \mathrm{~S}$ preparations. The apcD spectrum is very similar to that of the wild-type; differences between the two spectra can be attributed to a lesser quality apcD preparation. The apcDF and apcF emission spectra were quite different, with the apcF spectrum displaying relatively more AP emission than $680 \mathrm{~nm}$ emission.

Boltzmann calculations were also carried out for the apcF and apcDF preparations. I made the assumption that an AP chromophore substituted for the missing $\beta 18$ chromophore. The results of the Boltzmann calculations were very similar to those for the apcDE/C186S and apcE/C186S preparations, with $57 \%$ of the emission originating from AP, $40 \%$ from $680 \mathrm{~nm}$ emitters, and $3 \%$ 


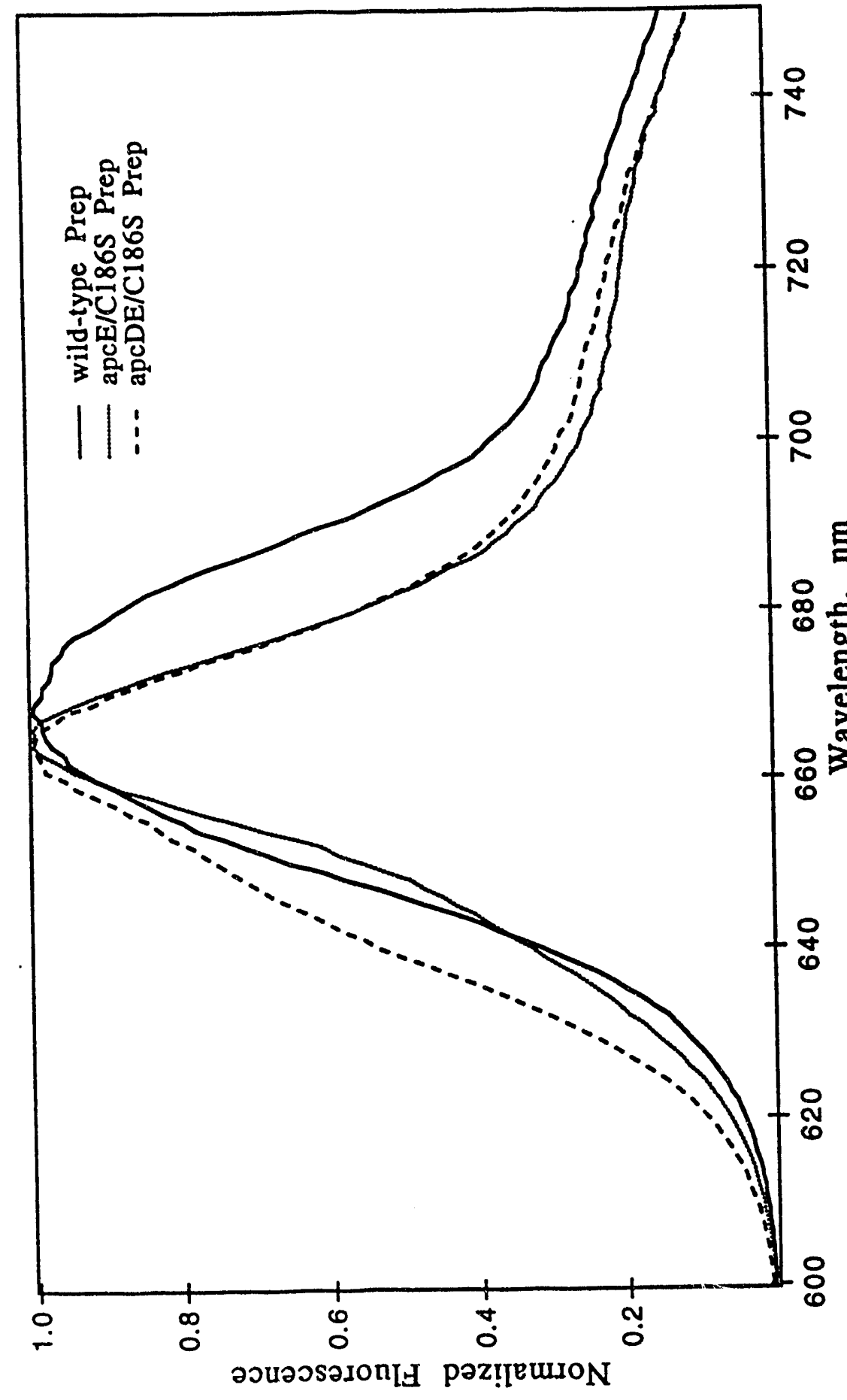

‡

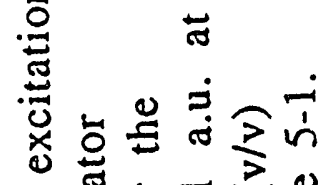

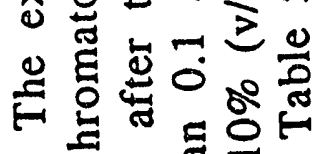
동 क 즈으

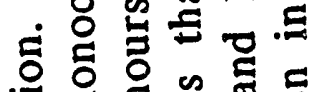

宅是嵒 ฮี

氙先 N ×.

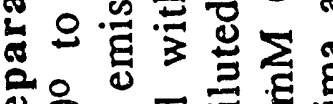
은웅

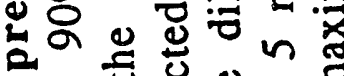

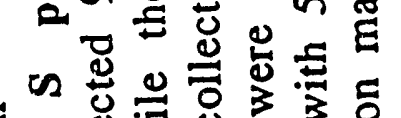

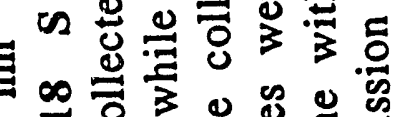

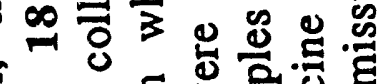

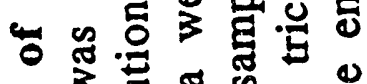
*

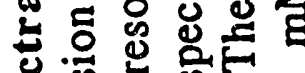

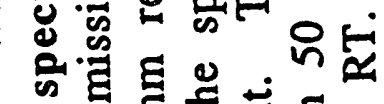
国苛标

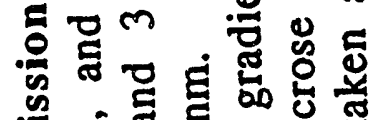
ह ส ส \%

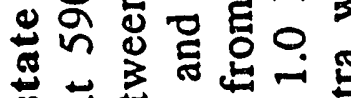
क䒕娄

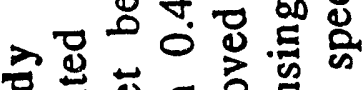
氙岛

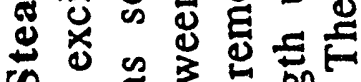

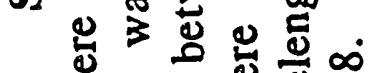

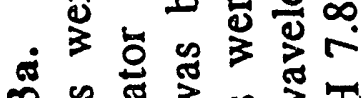

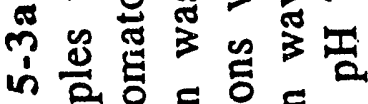

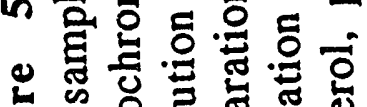
« \&

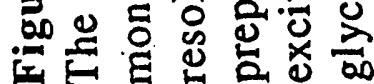




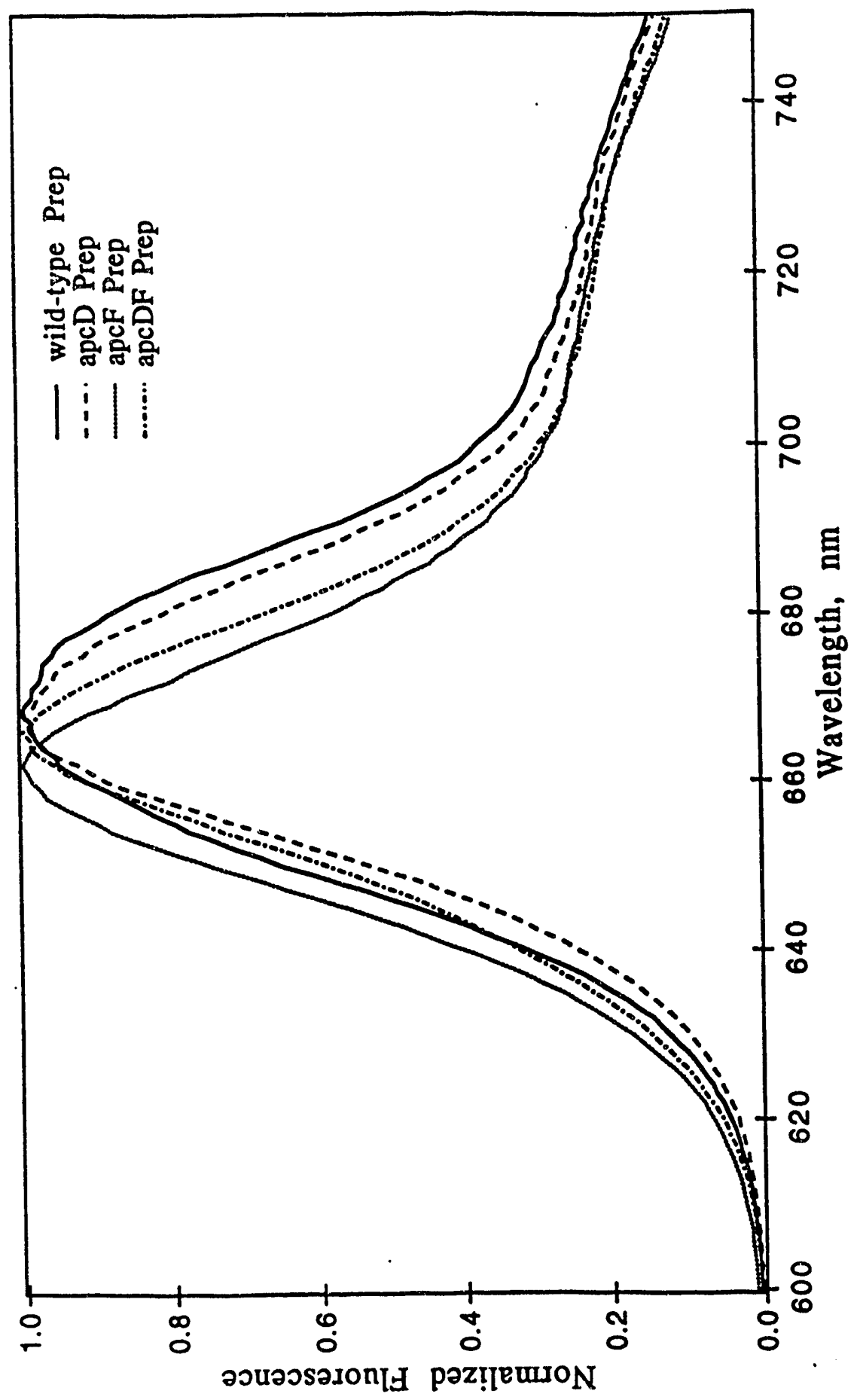

๕

苞 क

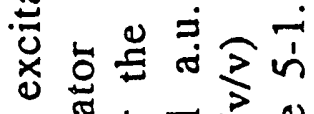

ข

총

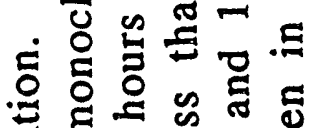

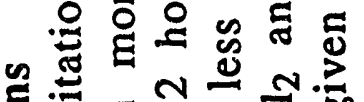

ปั.

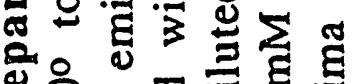

용

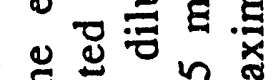

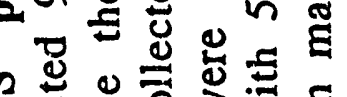

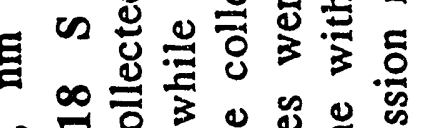

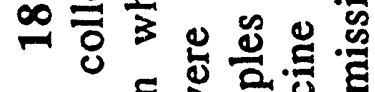

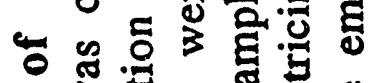

3 苟

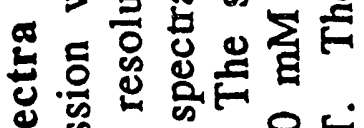

品落 ฮ国苞和

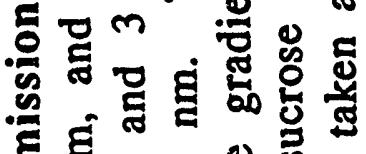

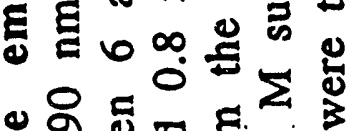

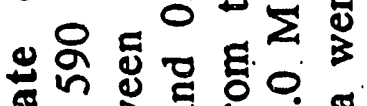

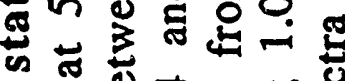

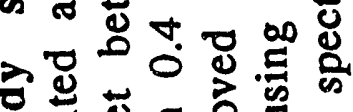

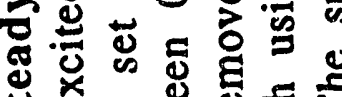

ङ

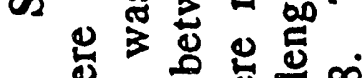

-

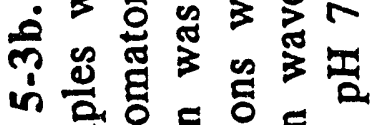
물을 을 흐.

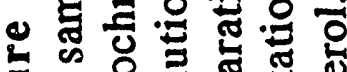
-

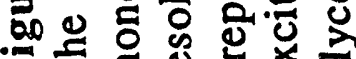
禹 E 
from PC. The apcDF spectrum qualitatively fits the calculations while the apcF spectrum displays too much PC emission.

\section{Low Temperature Emission}

AT $77 \mathrm{~K}$ back transfer between different chromophore pools within the $18 \mathrm{~S}$ preparation is very unlikely. All of the emission should originate from the lowest energy chromophores if the chromophores are coupled for energy transfer. I assume that freezing the $18 \mathrm{~S}$ preparations does not cause any significant structural changes within the complex such that energy transfer is impaired. As I mentioned before, the only change I expect to see is that the number of populated energy levels will decrease at $77 \mathrm{~K}$ relative to room temperature. Both the emission and absorption bands narrow. I froze the $18 \mathrm{~S}$ preparations in the same way I froze the intact PBS.

In Figure 5-4a,4b I have displayed the $77 \mathrm{~K}$ emission spectra obtained for the $18 \mathrm{~S}$ preparations. All of the spectra contain two different emission bands; one from PC at about $650 \mathrm{~nm}$ with a second one from the lowest energy chromophore present at 670 to $683 \mathrm{~nm}$. The emission maxima are given in Table 5-1. I found the apcD and wild-type spectra to be indistinguishable as were the apcDE/C186S and apcE/C186S spectra. The apcDF and apcF spectra are not identical.

Starting with Figure 5-4a, the main band of emission in the apcDE/C186S and apcE/C186S spectra is considerably blue-shifted from that of the wild-type, at $671 \mathrm{~nm}$ compared to $682 \mathrm{~nm}$. The apcDE/C186S and apcE/C186S preparations are missing the $L_{c m}$ 


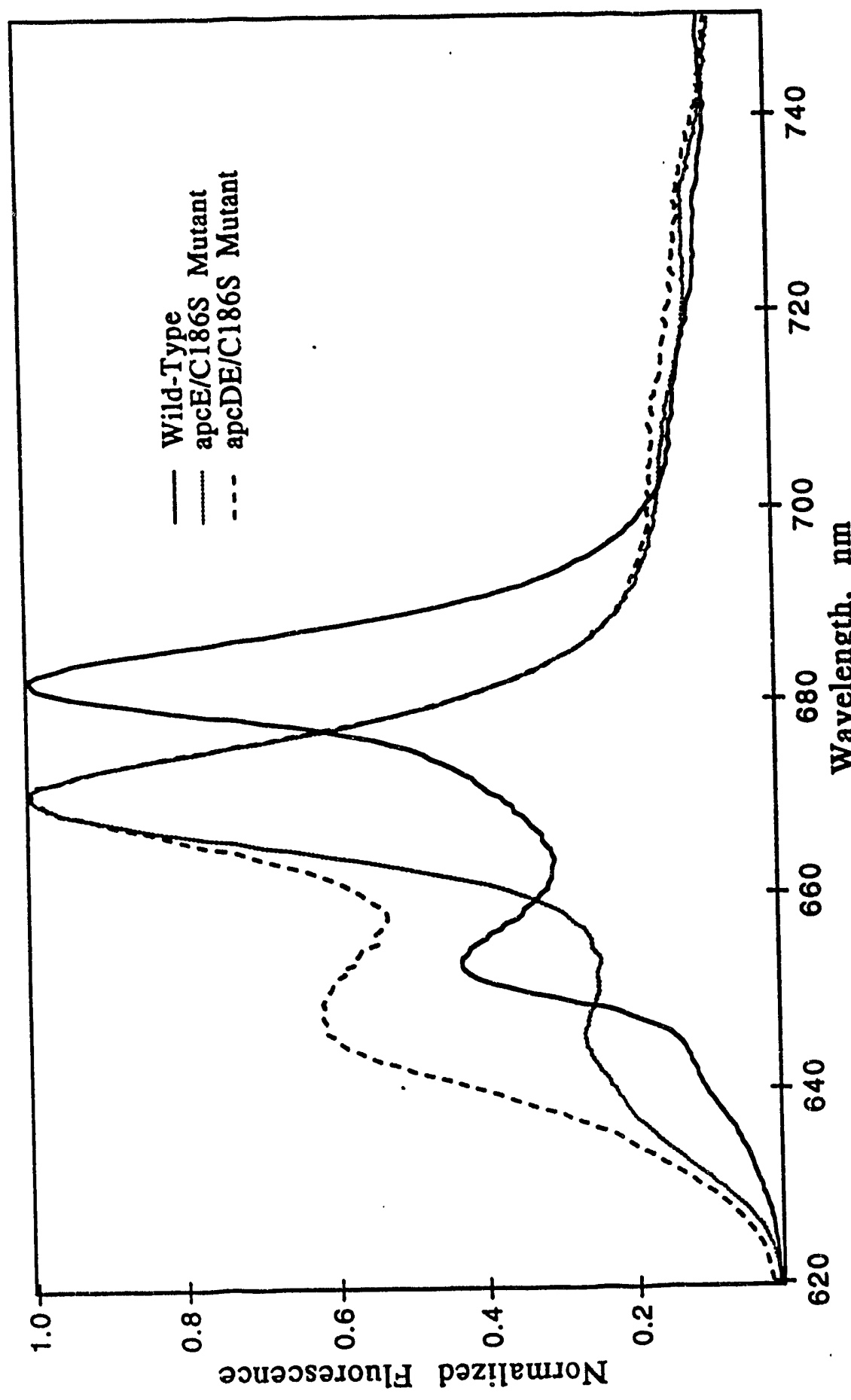

.

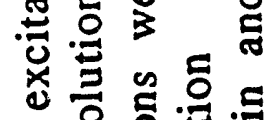

0 \& F

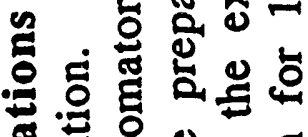
递总 종 क. 엉 응 닐

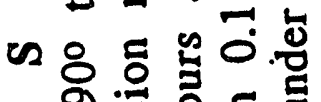

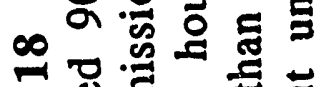
엉 过 $m$ 릉 - $\frac{0}{0}$ 옹

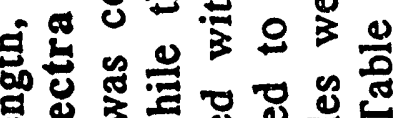
엉 $3 \frac{2}{2}$ 过

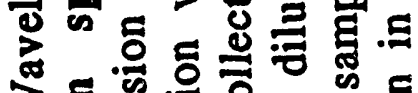
3 퉁 -율 \& $\$$ 형 렬

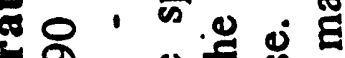
ڤ

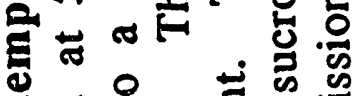

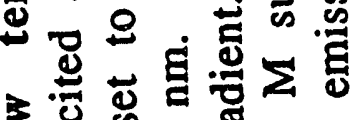
엉 क - 1 \& 0 क

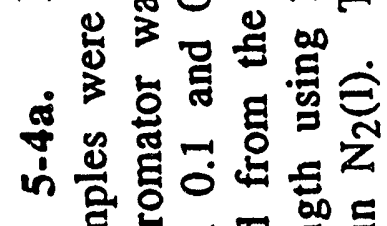

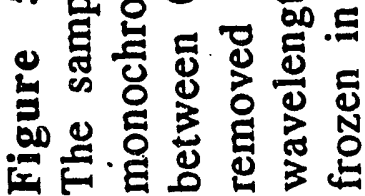




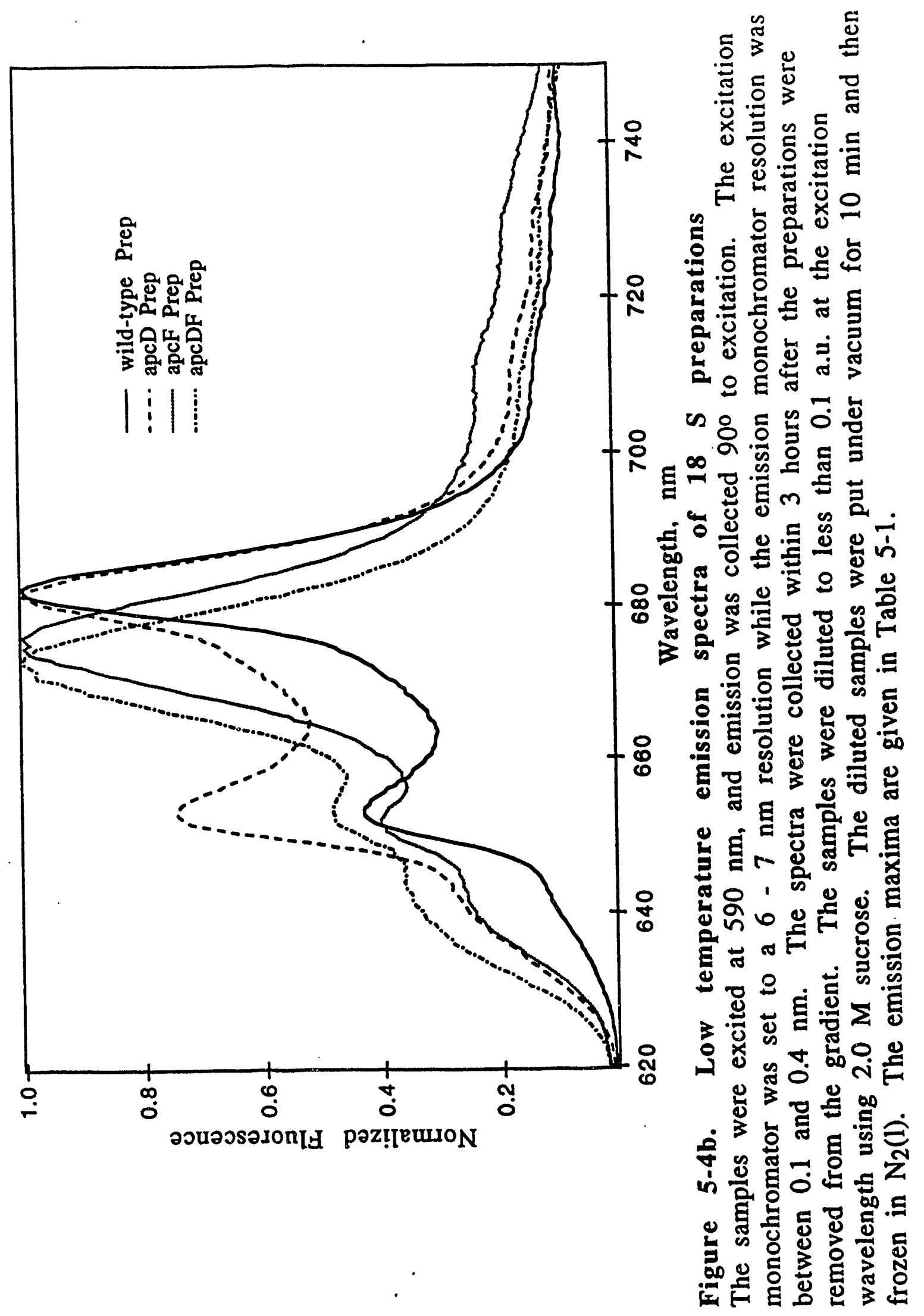


chromophore. Any emission coming from the complex should originate from the $\beta^{18}$ chromophore, the lowest energy chromophore remaining. In the last chapter, I assigned the $\beta^{18}$ chromophore to have an emission maximum at $678 \mathrm{~nm}$; this was in intact PBS from the apcDE/C186S cells which contained the modified chromophore. One must infer that the $\beta^{18}$ chromophore is very sensitive to the integrity of the PBS core.

The apcDF and apcF emission spectra shown in Figure 5-4b also display a blue-shifted emission band compared to that of the wild-type; the apcDF preparation emits at $673 \mathrm{~nm}$ while the apcF preparation emits at $676 \mathrm{~nm}$. This is the opposite of what was found with the room temperature emission spectra where the apcDF preparation emits further red than that of the apcF. The apcF preparation also does not contain any greater relative amounts of PC as shown by the height of the emission band at $653 \mathrm{~nm}$. Once again, one can only come to the conclusion that the assumption that the apcF and apcDF preparations have the same composition is false.

From the apcF spectrum, the main emission band is shifted about $6 \mathrm{~nm}$ further blue than in the wild-type spectrum. In the apcF preparation the $\beta^{18}$ chromophore is missing and probably substituted with an AP chromophore. Therefore, the lowest energy chromophore present would be the $\mathrm{L}_{\mathrm{cm}}$ chromophore; in the apcF preparation the $\mathrm{L}_{\mathrm{cm}}$ chromophore emits at $676 \mathrm{~nm}$. This is also further blue-shifted than expected. 


\section{Time-Resolved Fluorescence Emission}

As with the PBS preparations, I obtained time-resolved fluorescence data for the mutant and wild-type preparations with the exception of the apcDE/C186S strain. The apcDE/C186S $18 \mathrm{~S}$ preparations never worked out well enough to obtain time-resolved data; either the yield was too low, or the preparation was too contaminated with PC. I would expect the time-resolved data obtained for the apcE/C186S $18 \mathrm{~S}$ preparation to be similar to that obtained for the apcDE/C186S preparation because their steady state spectral properties have been indistinguishable up to this point.

As with the intact PBS, I expect to see emission not only from the terminal emitters in the preparation but also from other chromophores present because energy transfer from terminal emitters to AP will occur at RT. As I discussed in the steady state RT emission section of this chapter, in the wild-type spectrum almost $40 \%$ of the emission originates from AP in the preparation. Since the AP chromophores are coupled to the $680 \mathrm{~nm}$ emitters, they will decay with the same time constant.

The important time constant obtained from this study is the time needed for the energy to equilibrate over the antenna complex (the risetime for emission to occur at the terminal emitter). In the intact wild-type PBS, I measured it to be 0.15 ns. Assuming that all the chromophores are still coupled, the equilibration time for the 18 $\mathrm{S}$ preparations should be shorter than $0.15 \mathrm{~ns}$. I expect this result because fewer chromophores are present in the core complex, and 
fewer transfer steps are needed to transfer the energy to the 580 nm emitters.

Decay-associated spectra were obtained for each of the $18 \mathrm{~S}$ core preparations and are presented in Figures 5-5a through 5-5e. The samples were excited at 633 or $634 \mathrm{~nm}$, and emission was collected for almost $12 \mathrm{~ns}$ after the exciting pulse. The decays were fit to a minimum of three coinponents for the wild-type, apcD, apcF, and apcDF preparations and four components for the apcE/C186S preparation.

As shown in Figure 5-5a, the wild-type $18 \mathrm{~S}$ preparation displays v $\llcorner$ ry similar kinetics to that of wild-type PBS, Figure 4-4a. The $18 \mathrm{~S}$ preparation appears to be a good model for siudying the energy transfer properties of PBS except that it is somewhat unstable. Time-resolved fluorescence work was carried out on Synechococcus $630118 \mathrm{~S}$ preparations by Sandström and others using single photon timing (Sandström, Gillbro, et al., 1988). Using global analysis for several different excitation wavelengths, they obtained four lifetimes of $22,60,1045$, and 1810 ps. The yield of the 22 ps component was very small for $640 \mathrm{~nm}$ excitation, which probably is why I didn't resolve this component in my fluorescence data. They also reported results that they obtained with $580 \mathrm{~nm}$ excitation and $675 \mathrm{~nm}$ emission. Without using global analysis, they resolved three components of $2.7,1.8$, and $0.05 \mathrm{~ns}$; lifetimes quite similar to the ones I obtained from global analysis for the wild-type preparation. They concluded that the "18 S preparation is a functional entity of the PBS in terms of energy transfer 


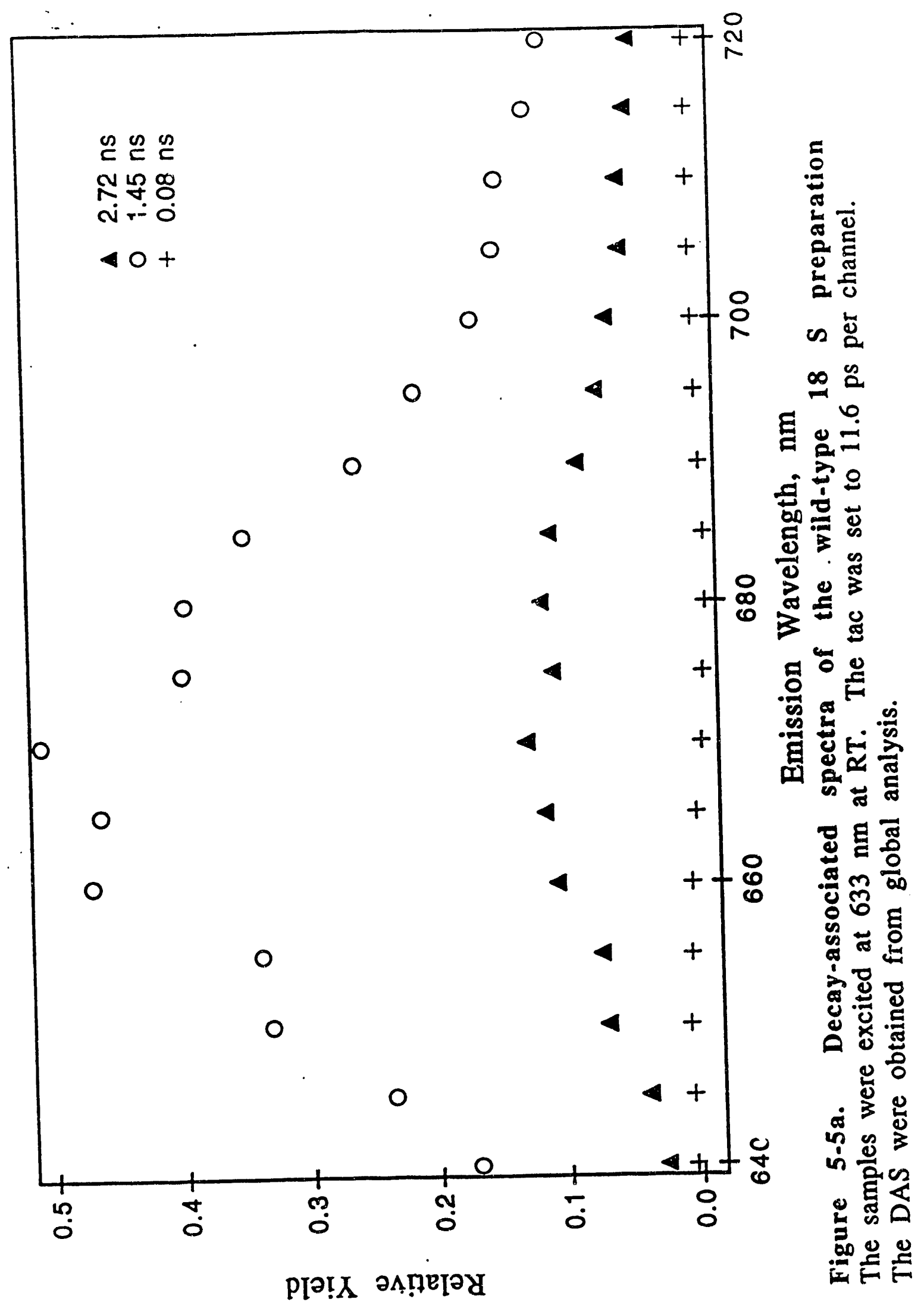




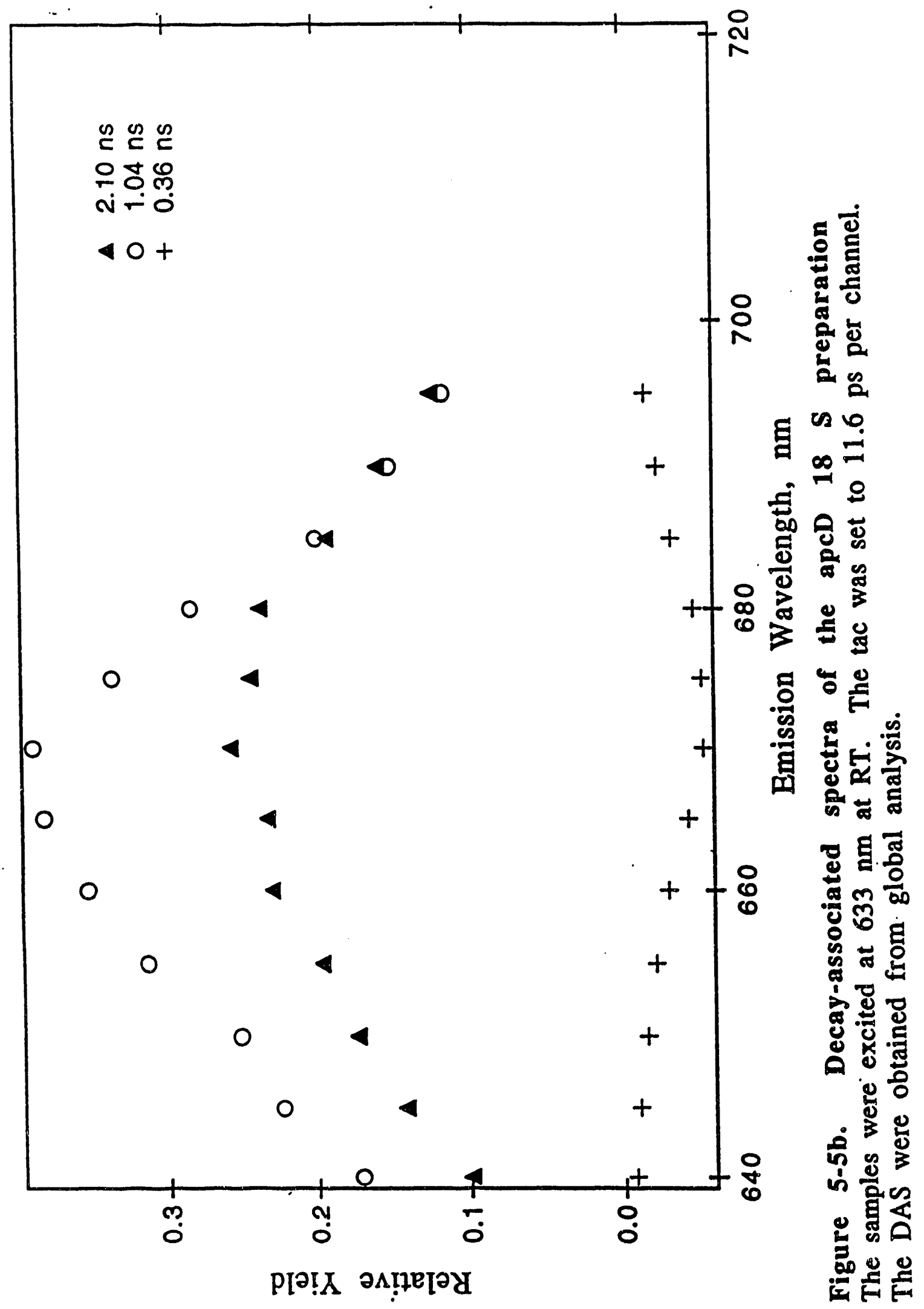


146

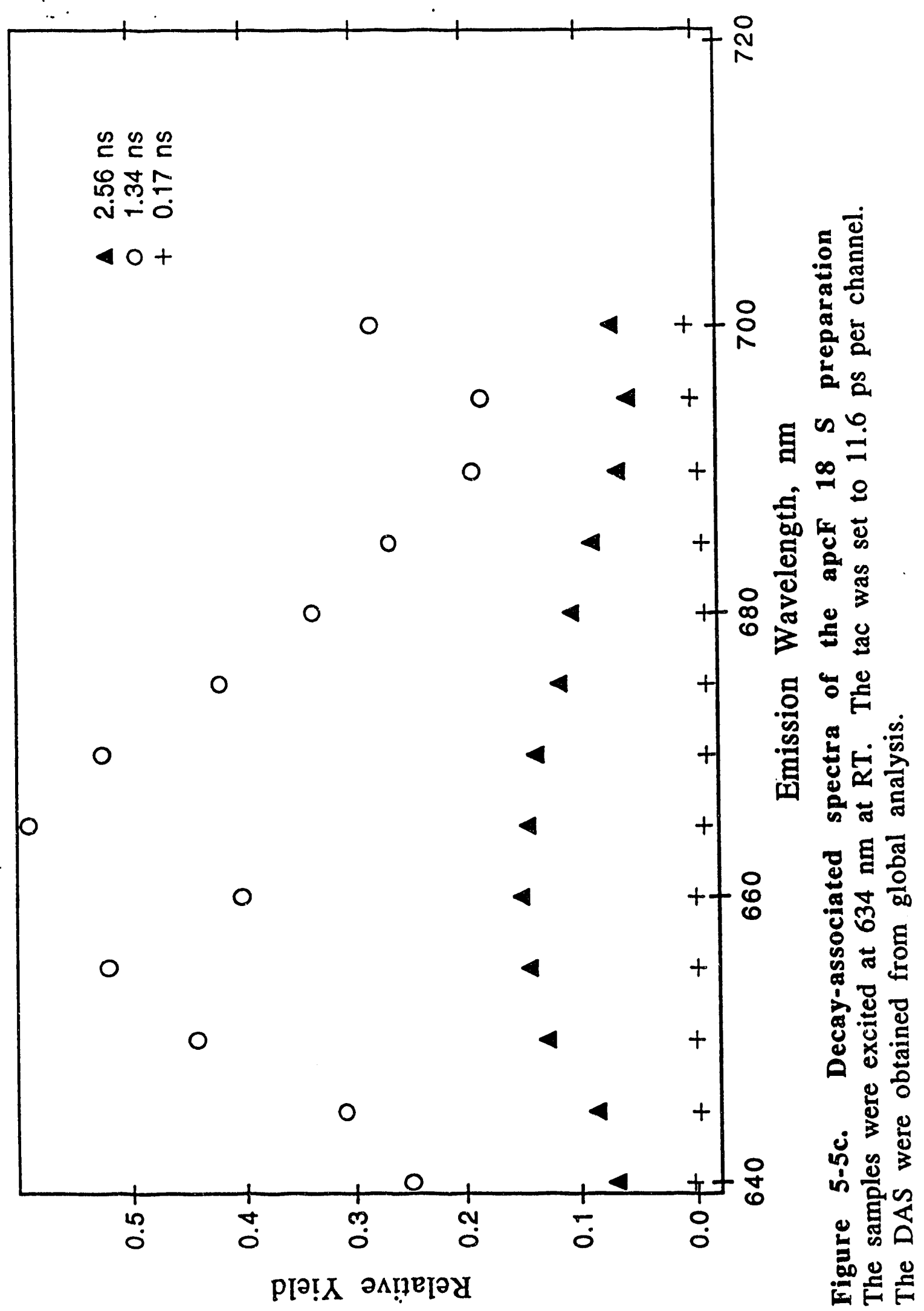




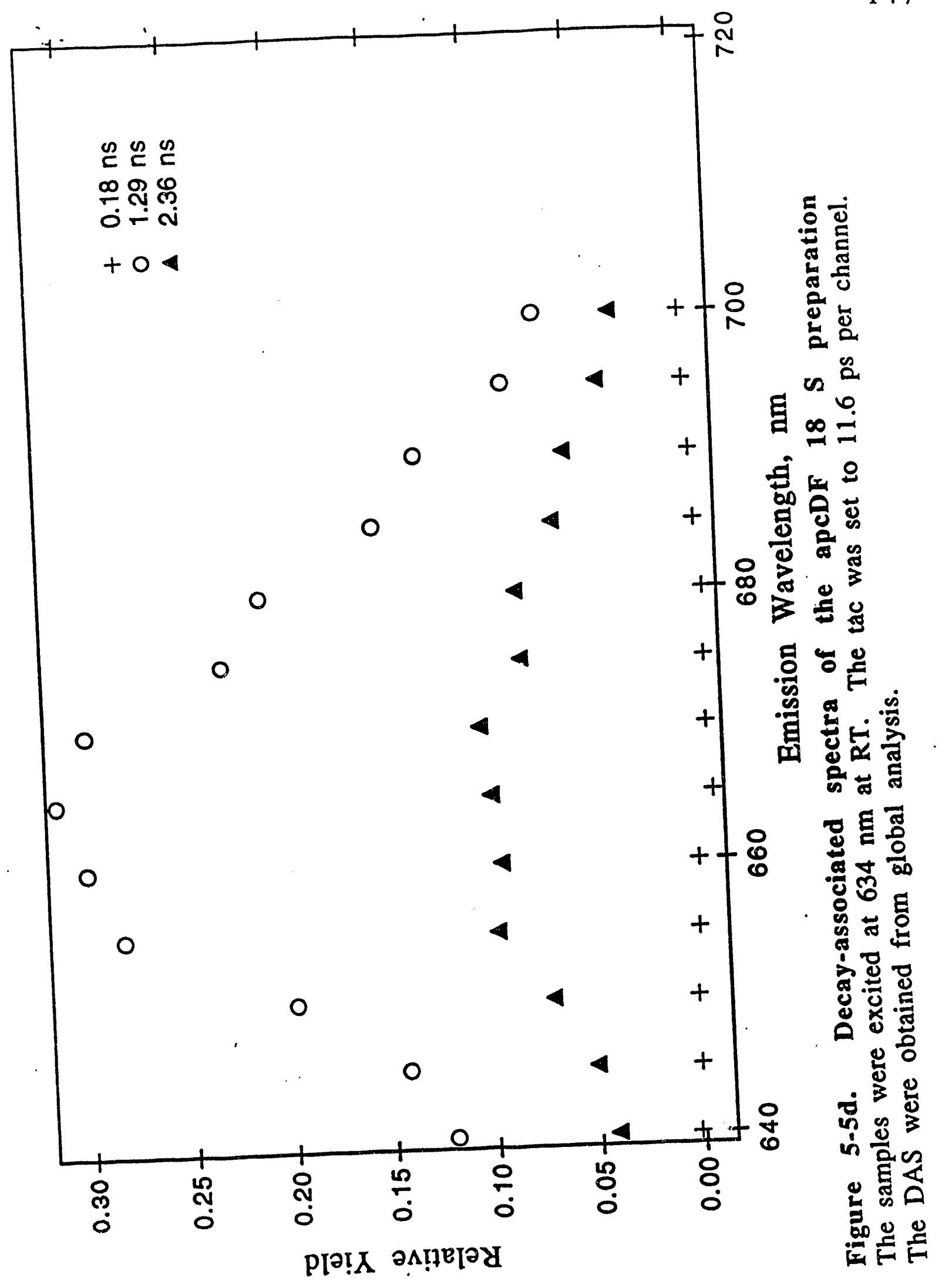




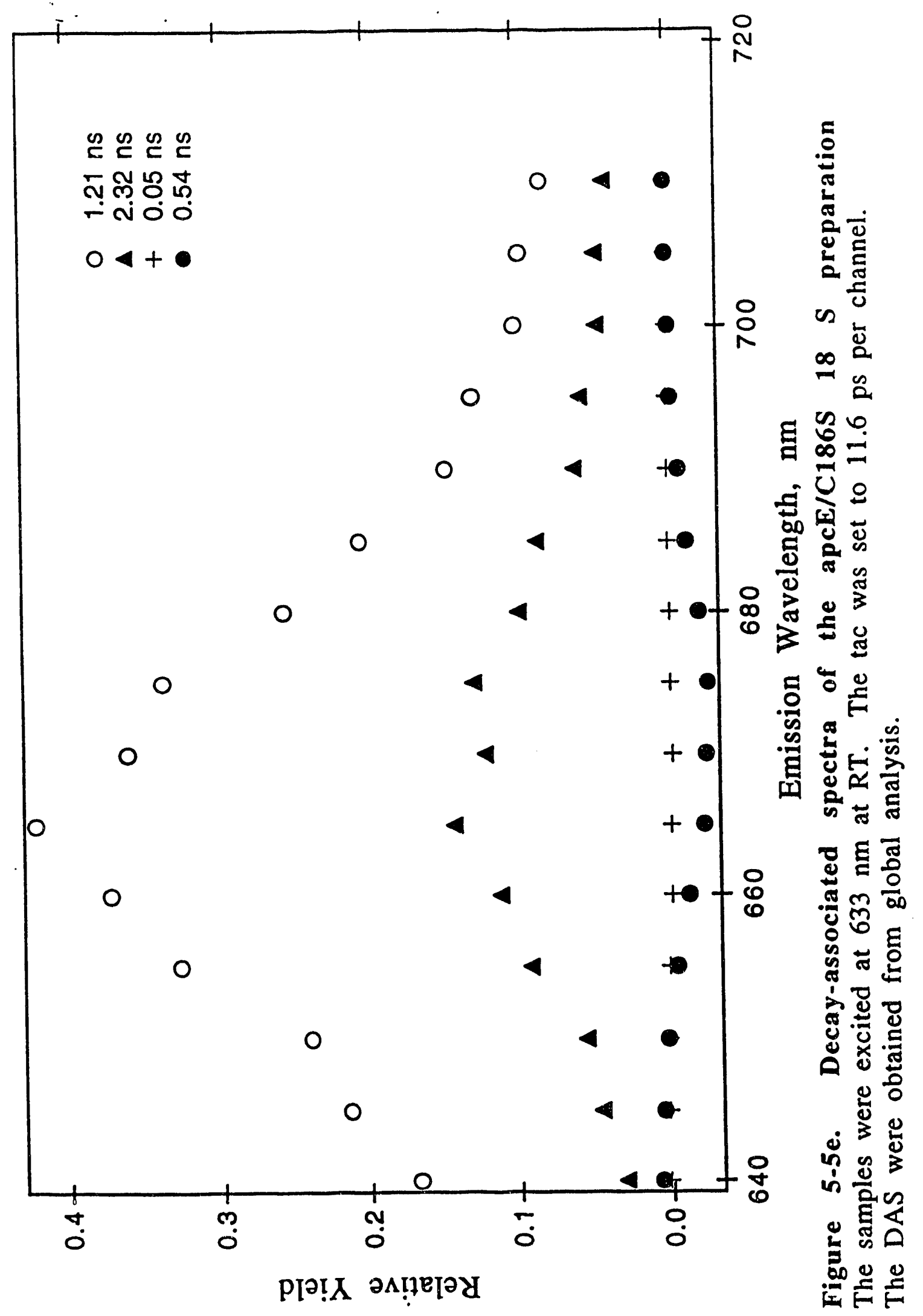


kinetics". I also found that the fluorescence decay data for the wild-type PBS and $18 \mathrm{~S}$ preparations are very similar.

For the wild-type preparation I resolve three components of $0.078 \mathrm{~ns}, 1.45 \mathrm{~ns}$, and $2.72 \mathrm{~ns}$, shown in Figure 5-5a. The $0.078 \mathrm{~ns}$ component corresponds to the time needed for the energy to be equilibrated over the complex. The longer lifetimes correspond to the fluorescence lifetimes of the terminal emitters along with the AP and PC chromophores coupled to the terminal emitters.

I found that the time-resolved emission data is very sensitive to sample integrity. If the sample is degraded such that the $\beta 18$ and $\mathrm{L}_{\mathrm{cm}}$ chromophores are no longer coupled, there is the appearance of $673 \mathrm{~nm}$ emission in the $77 \mathrm{~K}$ emission spectra. The samples which display the $673 \mathrm{~nm}$ emission also have DAS in which the shortest lifetime component is longer the usual. This is illustrated with the apcD DAS in Figure 5-5b; the equilibration time is more than four times that found in the wild-type DAS. This longer lifetime is characteristic of the degraded samples. This component also has a unique spectrum because it has only negative amplitude. The unique spectrum makes time-resolved fluorescence a good diagnostic tool to determine the integrity of $18 \mathrm{~S}$ preparations.

If the chromophores within the complex are in a different structural organization, or if the complex is partially dissociated, it will take longer for energy to travel from PC to the lowest energy chromophores because angles between transition dipoles or distances between chromophores are altered. Therefore, it will take longer for the energy to equilibrate over the complex, and the measured risetimes will be longer. Also, if the complex is not as 
intact structurally as the wild-type, the lifetimes of the chromophores could decrease if the chromophores are not being held in optimal conformations. Therefore, by comparing both the risetimes and the decay times of the chromophores from the $18 \mathrm{~S}$ preparations of the wild-type and the mutants, one can get some idea of the structural integrity of the complex along with the degree of coupling between the chromophores.

The DAS obtained for the apcDF and apcF $18 \mathrm{~S}$ preparations are indistinguishable from each other. Both sets of decays required three components of approximately $2.4,1.3$, and $0.18 \mathrm{~ns}$. The two longer components are slightly shorter than those obtained for the wild-type preparations, and the shortest component is twice as long as that for the wild-type.

The DAS obtained for the apcE/C186S preparation is shown in Figure 5-5e; a fourth decay component is always necessary to fit the decay data. The two longest decay components obtained for the apcE/C186S data are shorter than that of the wild-type, with lifetimes of 2.3 and $1.2 \mathrm{~ns}$ versus 2.7 and $1.45 \mathrm{~ns}$.

The two shortest lifetime components resolved in the apcE/C186S data are $0.05 \mathrm{~ns}$ and $0.5 \mathrm{~ns}$; both components have negative amplitudes from 660 to $680 \mathrm{~nm}$. I don't believe that the $0.5 \mathrm{~ns}$ component is due to degraded sample because the component does have positive amplitude from 640 to $650 \mathrm{~nm}$, unlike the $0.36 \mathrm{~ns}$ component found in the apcD DAS. 


\section{Discussion}

The study of the $18 \mathrm{~S}$ preparations has proved to be a worthwhile task. I was successful in obtaining a subcore preparation of the PBS which is missing the $\alpha$ AP-B subunit and most of the PC as shown by HPLC. The apcD and wild-type preparations and the apcE/C186S and apcDE/C186S preparations were indistinguishable as from each as expected. The spectral data on the $\mathrm{apcF}$ and $\mathrm{apcDF}$ preparations is harder to interpret because the apcF and apcDF preparations have different absorption and emission spectra. This probably means that the apcF and apcDF preparations have different compositions.

\section{The apcD Preparation}

As expected, the apcD preparation is virtually indistinguishable from that of the wild-type. The absorption and emission spectra from the apcD preparation are very similar to those of the wildtype. The small differences in the spectra can be attributed to experimental variability. The apcD and wild-type preparations appear to be of the same composition as expected.

\section{The apcF and apcDF Preparations}

The apcF and apcDF preparations were also expected to be of the same composition, but I found significant differences between their spectral data. The absorption spectra for the apcDF and apcF preparations are similar; differences between the absorption spectra can be attributed to differing amounts of PC present. The 
apcF and apcDF spectra are blue-shifted from that of the wild-type by 1 or $2 \mathrm{~nm}$.

The calculated difference spectra for the mutants are distorted somewhat because of differing amounts of PC are present in each preparation. I made the assumption that the number of chromophores present in each preparation is constant, and the area under the difference spectrum must equal zero. Because the mutants appear to contain more PC chromophores the difference spectrum is distorted with negative peaks appearing at 580 and $630 \mathrm{~nm}$. To compensate for the negative peaks, the difference spectra contain positive peaks at about $650 \mathrm{~nm}$ to make the net area difference equal to zero.

The apcDF difference spectrum contains a positive peak at about $680 \mathrm{~nm}$; the preparation from the mutant absorbs less at $680 \mathrm{~nm}$. The $680 \mathrm{~nm}$ peak is hidden under a large $660 \mathrm{~nm}$ peak in the apcF difference spectrum, but the peak does show up in the second derivative of the apcF difference spectrum. The large $660 \mathrm{~nm}$ peak in the apcF difference spectrum indicates that the changes within the apcF PBS core are probably not additive as assumed.

The $\beta^{18}$ subunit was expected to absorb at approximately $670 \mathrm{~nm}$. If the $\beta^{18}$ polypeptide was just omitted from the core without any further changes, the peak in the difference is redshifted and smaller in magnitude than expected. The difference spectra for the apcF and apcDF preparations can be explained if the $\beta^{18}$ subunit is substituted with a bluer absorbing protein. The higher energy chromophore will partially compensate for the lack of $\beta^{18}$ chromophore absorption at higher energies. At lower 
energies, the bluer-absorbing chromophore can no longer compensate for the lack of the $\beta^{18}$ absorption, and this leads to a hole in the absorption spectrum. The substitution would also lead to smaller absorption differences.

The replacement of the $\beta^{18}$ subunit with a $\beta^{A P}$ is a reasonable hypothesis based on the similarity in the amino acid sequences of the two proteins. In Mastigocladus laminosus, Füglistaller, Rümbeli et al. found the first 138 amino acids of the $\beta^{18}$ and $\beta A P$ subunits to have $62 \%$ homology (Füglistaller, Rümbeli et al, 1984).

The RT emission spectra for the apcDF and apcF preparations are blue-shifted from that of the wild-type as expected. More emission originates from the AP chromophores in the preparations from the mutants as expected. The apcDF and apcF preparations are missing a $680 \mathrm{~nm}$ emitter.

The RT emission spectrum of the apcDF preparation qualitatively fit the results obtained from Boltzmann calculations while the apcF spectrum displays too much PC emission. All of the apcF emission spectra I have obtained display larger amounts of PC emission. The apcF emission is consistently blue-shifted from that of the apcDF preparations. Apparently, the apcF and apcDF preparations have different polypeptide compositions. The PBS core did not dissociate in the same way for the apcF and apcDF mutants.

The low-temperature emission spectra from the apcF and apcDF preparations also display a blue-shifted emission band compared to that of the wild-type preparation. Unlike the RT emission spectrum, the low-temperature emission spectrum of the apcF 
preparation is red-shifted from that of the apcDF preparation. This result also provides further evidence that the apcF and apcDF preparations are of different composition, and that the apcF preparation contains larger numbers of PC chromophores. PC emission contributes a large amount of the total fluorescence yield at RT and shifts the emission maximum blue in the apcF spectrum.

From the low-temperature emission data one can assign emission maximum to some of the chromophores within the PBS core. The apcF preparation contains only one $680 \mathrm{~nm}$ emitter, the $\mathrm{L}_{\mathrm{cm}}$ chromophore. In the apcF preparation, the $\mathrm{L}_{\mathrm{cm}}$ chromophore emits at $676 \mathrm{~nm}$. The $676 \mathrm{~nm}$ emission maximum is $7 \mathrm{~nm}$ bluer than what is seen with the wild-type preparation. The blue-shift is caused by the lack of the $\beta^{18}$ subunit.

The time-resolved data for the apcDF and apcF preparations were indistinguishable and slightly modified from that of the wildtype preparation. The two longest decay components resolved are shorter than those found in the wild-type DAS. The decrease in the lifetimes of the emitting chromophores indicates a larger degree of structural disorder within the apcF and apcDF preparations. The chromophores are no longer being held in optimal conformations, and more nonradiative deactivation occurs.

The shortest decay component which corresponds to the equilibration time necessary for the energy to travel throughout the complex is longer in the mutants than in the wild-type. A longer equilibration time indicates impaired energy transfer within the complex. 
In summary, the apcF and apcDF preparations have different polypeptide compositions. The apcF preparation contains larger amounts of PC. Both the apcF and apcDF preparations appear to have a higher energy chromophore substituting for the missing $\beta^{18}$ chromophore as shown in the absorption difference spectrum. From the low-temperature emission spectra, one can assign the $\mathrm{L}_{\mathrm{cm}}$ chromophore to have an emission of $676 \mathrm{~nm}$ in the absence of the $\beta 18$ subunit. The time-resolved emission data indicate that the preparations from the mutants are more disordered, and that energy transfer within the preparation is impaired.

\section{The apcE/C186S and apcDE/C186S Mutants}

As I mentioned earlier, the modified chromophore present in the apcE/C186S and apcDE/C186S PBS is missing from the $18 \mathrm{~S}$ preparations. The apcE/C186S and apcDE/C186S preparations were virtually indistinguishable spectrally, except the apcDE/C186S preparation had larger amounts of PC present. Absorption spectra from the mutants overlaid exactly above $650 \mathrm{~nm}$; both spectra displayed a large absorption decrease compared the wild-type preparation at about $670 \mathrm{~nm}$. From the absorption data, I assign the $\mathrm{L}_{\mathrm{cm}}$ chromophore to absorb maximally at $670 \mathrm{~nm}$.

The RT emission spectra for the apcE/C186S and apcDE/C186S preparations are not well described by the Boltzmann calculations. The apcE/C186S and apcDE/C186S emission spectra contain too much $\mathrm{AP}$ and $\mathrm{PC}$ emission. In the Boltzmann calculations, I assumed that the $\beta^{18}$ chromophore emitted at $680 \mathrm{~nm}$. If the $\beta^{18}$ chromophore emits at a higher energy than expected, more 
emission would originate from PC and AP chromophores. I redid the Boltzmann calculations for the RT emission using an energy level for the $\beta^{18}$ chromophore that corresponds to $670 \mathrm{~nm}$ instead of $679 \mathrm{~nm}$. I used the low-temperature emission data to choose the new energy level for the $\beta^{18}$ chromophore. With the new energy level, I calculate that only $22 \%$ of the emission should originate from the $\beta^{18}$ chromophore while AP will contribute $75 \%$. The revised Boltzmann calculations fit the experimental data from the apcE/C186S preparation. The apcDE/C186S spectrum still contains too much PC emission.

The low-temperature emission spectra for the apcDE/C186S and apcE/C186S preparations are indistinguishable with both spectra considerably blue-shifted from that of the wild-type. The $11 \mathrm{~nm}$ blue-shift in the apcDE/C186S and apcE/C186S spectra can be attributed to the absence of the $\mathrm{L}_{\mathrm{cm}}$ chromophore. In the apcDE/C186S and apcE/C186S preparations, the lowest energy chromophore remaining is the $\beta^{18}$ chromophore which emits at $671 \mathrm{~nm}$ in the absence of the $\mathrm{L}_{\mathrm{cm}}$ chromophore.

The time-resolved emission from the apcE/C186S preparations is quite different from that of the wild-type preparation. The apcE/C186S emission decay is more complicated and requires the addition of a fourth lifetime component in the fit. The two longer lifetimes which I assign to coupled PC, AP, and the terminal emitters are shorter than those seen in the wild-type DAS. As with the apcDF and apcF preparations, I ascribe the shortening of the coupled chromophore decay times to greater disorder within the preparation. 
The additional fast decay component in the apcE/C186S DAS is possibly due to the slowing down of a energy transfer step within the core preparation. Unlike DAS obtained for partially dissociated preparations, both of the fast decay components have positive amplitude in the blue part of the emission spectrum. I don't think either component is due to dissociated sample.

There are two possible explanations for the additional decay component resolved in the apcE/C186S DAS. One possibility is that an energy transfer step, such as transfer from PC to AP, apparently is slowed in the apcE/C186S mutant. The transfer step can now be detected with single photon timing. In this case, the additional lifetime would be the $0.050 \mathrm{~ns}$ component and the $0.54 \mathrm{~ns}$ lifetime would correspond to the 0.08 ns lifetime from the wild-type DAS. The time necessary for the energy to equilibrate over the antenna is lengthened by a factor of 5 .

There is a second possible explanation for the additional component in the spcE/C186S DAS. In the wild-type DAS two decay components very similar in size are not being resolved from each other. In the apcE/C186S DAS, one of the components is sufficiently difrerent such that it now can be resolved from the second component. In this case, the $0.05 \mathrm{~ns}$ lifetime is probably unchanged while the $0.54 \mathrm{~ns}$ lifetime component is actually lengthened by a factor of 5 .

The take home message from the apcE/C186S time-resolved data is that the chromophores within the preparation are more disordered and that energy transfer within the core is impaired in some manner. 
The apcDE/C186S and apcE/C186S preparations are indistinguishable spectrally except that the apcDE/C186S preparations typically contain larger amounts of PC contamination. As shown by the absorption spectra, the $\mathrm{L}_{\mathrm{cm}}$ chromophore absorbs at $670 \mathrm{~nm}$. Also, the $\beta^{18}$ chromophore emits at an energy greater than $680 \mathrm{~nm}$ as shown both by the Boltzmann calculations for the RT emission and by the $77 \mathrm{~K}$ emission spectrum. The time-resolved emission data indicates that the chromophores within the apcE/C186S $18 \mathrm{~S}$ preparations are more disordered, and energy transfer within the complex is impaired.

\section{Conclusions}

Using the low temperature emission spectra of apcDE/C186S PBS, I assigned the emission maximum of the $\beta^{18}$ chromophore to 679 nm. When the $\mathrm{L}_{\mathrm{cm}}$ chromophore is missing from the PBS core, the $\beta 18$ chromophore emits at $671 \mathrm{~nm}$, as shown by the lowtemperature emission data from apcE/C186S $18 \mathrm{~S}$ preparations. If the $\beta^{18}$ subunit is missing from the intact $P B S$, the $L_{c m}$ chromophore emits at $682 \mathrm{~nm}$ (from the $77 \mathrm{~K}$ emission spectra of apcF and apcDF PBS). In the $18 \mathrm{~S}$ preparations, the $\mathrm{L}_{\mathrm{cm}}$ chromophore emits at 676 $\mathrm{nm}$ (from the $77 \mathrm{~K}$ emission spectrum of the apcF preparation).

I have plotted several low-temperature emission spectra of wild-type preparations in Figure 5-6. Some of the preparations are partially dissociated as shown by the present of an emission band at about $673 \mathrm{~nm}$ along with a corresponding decrease in the 682 $\mathrm{nm}$ emission. The appcarance of $673 \mathrm{~nm}$ emission could be due to several factors. The $\mathrm{L}_{\mathrm{cm}}$ polypeptide could be partially degraded 


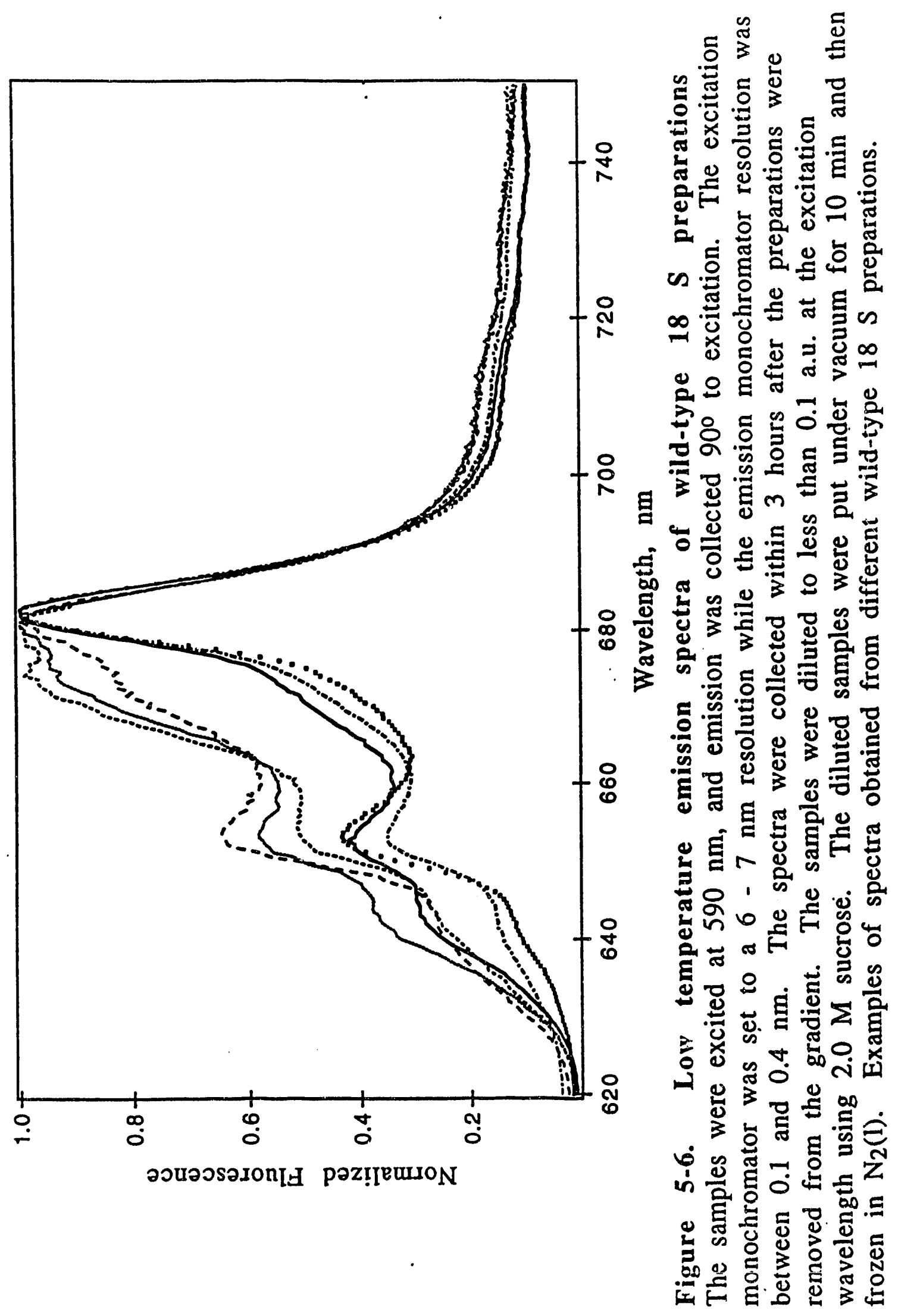


from proteases present; I have noticed that the $18 \mathrm{~S}$ preparations degrade much faster if protease inhibitor is not added to the buffers. Isono and Katoh also reported that a core preparation they studied was much more susceptible to chrymotrypsin than intact PBS (Isono and Katoh, 1987). The $673 \mathrm{~nm}$ emission could be due to partial dissociation of the preparation; the $\beta^{18}$ and $L_{c m}$ chromophore could be coming uncoupled. The $673 \mathrm{~nm}$ emission could also be from the $\beta^{18}$ chromophore if energy transfer is impaired between the $\beta 18$ and the $L_{c m}$ chromophores.

The point $I$ am trying to make with all this data is that there is an interaction between the $\beta^{18}$ subunit and the $L_{c m}$ chromophore. Both the $L_{c m}$ chromophore and the $\beta^{18}$ subunit need to be present to obtain the $683 \mathrm{~nm}$ emission seen with both the wild-type PBS and $18 \mathrm{~S}$ preparation. The interaction is possibly structural in nature. The $\beta$ AP subunit, which might substitute for the $\beta^{18}$ subunit, has a very different $\mathrm{C}$-terminal end; the last 15 amino acids of the $\beta^{18}$ subunit are more negatively charged than the amino acids from the $\beta A P$ subunit (Bryant, 1988). The C-terminal end of the $\beta^{18}$ subunit could interact with the $L_{c m}$ polypeptide to stabilize the chromophore. This interaction becomes more important when the PBS core is partially dissociated as in the $18 \mathrm{~S}$ preparations.

The $\beta^{18}$ and $L_{\mathrm{cm}}$ chromophores are probably close in space. The $\beta 18$ chromophore appears to be very sensitive to the absence of the $\mathrm{L}_{\mathrm{cm}}$ chromophore, and it's emission maximum shifts more than $8 \mathrm{~nm}$ if the $\mathrm{L}_{\mathrm{cm}}$ chromophore is missing. I feel that this large of a 
blue-shift in the emission spectrum has to mean that the $\beta^{18}$ chromophore is right next to the $\mathrm{L}_{\mathrm{cm}}$ chromophore binding pocket. I will sum up all my conclusions in the next chapter, and I will propose some future projects. 


\section{References for Chapter 5}

Bryant, D. A. (1988). Genetic analysis of phycobilisome biosynthesis, assembly, structure, and function in the cyanobacterium

Synechococcus sp. PCC 7002, In S. E. Stevens, Jr., and D. A. Bryant (eds.) Light-Energy Transduction in Photosynthesis: Higher Plant and Bacterial Models. The American Society of Plant Physiologists, Rockville, MD (USA) 62-93.

Füglistaller, P., R. Rümbeli, F. Suter, and H. Zuber (1984). HoppeSeyler Z Physiol Chem, 365, 1085-1096.

Gingrich, J. C., D. J. Lundell, and A. N. Glazer (1983). J. Cell. Biochem. 22: $1-14$

Isono, T. and T. Katoh (1987). Arch. Biochem. Biophys. 256: 317324.

Lundell, D. J. and A. N. Glazer (1983). J. Biol. Chem. 258: 894-901.

Sandström, A., T. Gillbro, V. Sundström, J. Wendler, and A. R. Holzwarth (1988). Biochim. Biophys. Acta 933: 54-64.

Swanson, R. V. and A. N. Glazer (1990). Anal. Biochem. 188: 295299.

Yamanaka, G., D. J. Lundell, and A. N. Glazer (1982). J. Biol. Chem. 257: $4077-4086$. 
Chapter 6. Conclusions and Future Work

In this chapter I will try to draw together all my results from the previous chapter and suggest some directions for future work on PBS. I have summarized all the spectral results in Table 6-1.

The $\alpha$ AP-B subunit

The deletion of the $\alpha$ AP-B subunit from the PBS core didn't create any dramatic changes in the workings of the antenna complex. The doubling time for the apcD cells is very similar to that of the wildtype under our growth conditions. Energy transfer to the PSII reaction center appears to be unimpaired as shown by timeresolved and steady state fluorescence studies on whole cells.

The deletion of the $\alpha^{A P-B}$ subunit from the PBS antenna had very little effect on the spectral properties of the isolated antenna with the exception of the RT emission spectrum. This result was seen both with the apcD and apcDF strains. The $\alpha$ AP-B subunit does contribute to the RT emission but not to the low temperature emission spectrum. The $\alpha^{\text {AP-B }}$ chromophore is slightly higher in energy than the $\mathrm{L}_{\mathrm{cm}}$ chromophore; at $77 \mathrm{~K}$, energy transfer to the $\mathrm{L}_{\mathrm{cm}}$ occurs rather than emission. At $\mathrm{RT}$, back transfer from the $\mathrm{L}_{\mathrm{cm}}$ occurs readily, and the $\alpha \mathrm{AP}-\mathrm{B}$ chromophore fluoresces.

The deletion of the $\alpha^{\text {AP-B }}$ subunit did not seem to perturb the emitting chromophores within the core as shown by the timeresolved emission measurements on the apcD and wild-type PBS. The decays lifetimes did not shorten. Maxson studied the apcD 
Table 6-1

Emission Maxima Assignments

Low Temperature

Chromophore Type Emission Maximum

$\alpha$ AP-B in Intact PBS

$\beta^{18}$ in Intact PBS

$\beta^{18}$ in Core Prep

$\mathbf{L}_{\mathrm{cm}}$ in Intact PBS

with $\beta^{18}$ present

$\mathbf{L}_{\mathrm{cm}}$ in Intact PBS

with $\beta^{18}$ missing

$\mathrm{L}_{\mathrm{cm}}$ in Core Prep

with $\beta^{18}$ present

$\mathrm{L}_{\mathrm{cm}}$ in Core Prep with $\beta^{18}$ missing
$682 \mathrm{~nm}$

$678 \mathrm{~nm}$

$671 \mathrm{~nm}$

$683 \mathrm{~nm}$

$682 \mathrm{~nm}$

$683 \mathrm{~nm}$

$676 \mathrm{~nm}$ 
strain earlier, and she reached similar conclusions (Maxson, Sauer, et al., 1989).

\section{The $\beta 18$ subunit}

The deletion of the $\beta^{18}$ subunit from the PBS antenna was found to have a larger effect than the deletion of the $\alpha$ AP-B subunit. The doubling times of the apcF and apcDF cells were slightly longer than that of the wild-type cells. From the absorption spectrum of the $18 \mathrm{~S}$ core preparation, it appears that the $\beta^{18}$ subunit is replaced by a $\beta A P$ subunit in both the apcF and apcDF mutants.

As with the $\alpha^{\text {AP-B }}$ chromophore, the $\beta^{18}$ chromophore contributes to the RT emission of the PBS but not to the low temperature emission. The $\beta^{18}$ chromophore is slightly higher in energy than both the $\alpha^{A P-B}$ and $L_{c m}$ chromophores. The $\beta^{18}$ chromophore transfers energy to the $\mathrm{L}_{\mathrm{cm}}$ at $77 \mathrm{~K}$.

The $\beta^{18}$ subunit interacts with the $L_{c m}$ chromophore as shown by the low-temperature emission spectra of the $18 \mathrm{~S}$ preparations. Both the $\beta^{18}$ subunit and the $L_{c m}$ chromophore are required for the PBS core to emit at $683 \mathrm{~nm}$. The type of interaction between the two chromophores is unclear at this time. It could be a structural interaction in which the $\beta^{18}$ subunit acts to stabilize the $L_{c m}$ chromophore. In this case, the chromophores would probably be close so that the removal of the chromophore from the $\mathrm{L}_{\mathrm{cm}}$ binding pocket perturbs the chromophore binding pocket of the $\beta 18$ subunit.

The $\beta^{18}$ and $L_{c m}$ chromophores could also be excitonically coupled. In this case, the removal of one of the chromophores 
would destroy the exciton states; this could explain the blue-shift of the low temperature emission from the apcF and apcDF PBS. I will discuss some possible experiments which could address this question later in the chapter.

The deletion of the $\beta^{18}$ from the PBS core caused some disorder within the PBS. The lifetimes of the coupled chromophores decreased significantly in both PBS and core preparations. Some of the chromophores within the core are not being held in their ideal conformations.

\section{The $L_{c m}$ Chromophore}

The substitution of a serine for cysteine 186 in the $L_{c m}$ proved to be a mutation resulting in qualitatively different behavior, as shown by the emission spectra from the apcE/C186S and apcDE/C186S mutants. The substitution of the cysteine with serine to alter the binding site of the $\mathrm{L}_{\mathrm{cm}}$ chromophore did not result in the absence of the chromophore from the PBS. Instead, the chromophore appears to be associated with the PBS complex in some noncovalent fashion. Since the chromophore isn't covalently bound to the protein, it probably is still a $3(\mathrm{Z})$-phycocyanobilin, the immediate precursor to the bound chromophore (Rhie and Beale, 1992). The 3(Z)-phycocyanobilin contains an additional double bond leading to increased $\pi$-orbital conjugation and altered spectroscopic properties. The modified chromophore was found to absorb at $695 \mathrm{~nm}$ and emit at. $715 \mathrm{~nm}$. 
The presence of the modified chromophore really impairs the growth of the apcDE/C186S and apcE/C186S cells. I found that the modified chromophore is at a lower energy than the reaction centers, so it diverts energy away from the reaction centers. It also acts to quench the PBS complex as shown by its unusually short decay lifetime. Large amounts of energy normally collected by the PBS for transfer to PSII is lost as emission or in nonradiative ways in the apcE/C186S and apcDE/C186S cells.

The modified chromophore is missing from the $18 \mathrm{~S}$ preparations for the apcDE/C186S and apcE/C186S cells. The absence of the $\mathrm{L}_{\mathrm{cm}}$ chromophore leaves a hole in the absorption spectrum of the preparation at approximately $670 \mathrm{~nm}$.

As with the deletion of the $\beta 18$ subunit, the modification to the $\mathrm{L}_{\mathrm{cm}}$ polypeptide perturbed the emitting chromophores within the PBS core. This was shown by the decrease in the decay lifetimes of both the intact PBS and the core preparations. The PBS core is somewhat disordered with some of the chromophors no longer in ideal conformations. 


\section{Energy Transfer within the Core}

Using the conclusions I made above, I propose that energy transfer within the PBS core occurs as outlined below.

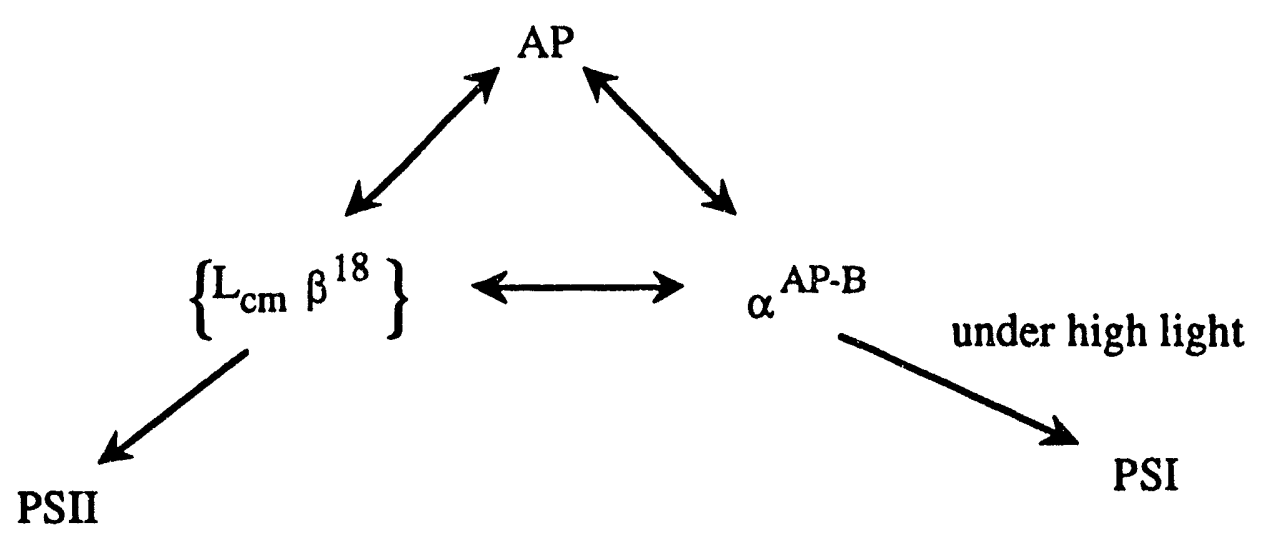

\section{Future Directions}

I still have quite a few unanswered questions that I would like to examine. First, what is the nature of the interaction between the $\mathrm{L}_{\mathrm{cm}}$ and $\beta 18$ chromophores. Are the chromophores excitonically coupled or just structurally dependent? One way to answer this question would be to create another mutant similar to the apcE/C186S mutant, change the cysteine on the $\beta^{18}$ subunit to serine to create a apcF/C84S mutant. In analogy to the apcE/C186S mutant, the chromophore associated with the $\beta^{18}$ polypeptide should be present in modified form in the intact PBS and missing in the $18 \mathrm{~S}$ preparation.

If the interaction between the $\beta^{18}$ subunit and the $L_{c m}$ chromophore is structural in nature, then the low temperature 
emission of PBS from the apcF/C84S mutant should be identical to that of the wild-type. Using the absorption spectrum from the $18 \mathrm{~S}$ preparation from this mutant, one could also gain information about the absorption of the $\beta^{18}$ chromophore.

Another interesting aspect to this study would be the creation of a apcDFE/C186S mutant with the PBS missing all of the unusual polypeptides. I have the feeling that the organism would grow very slowly, but it would probably have some very interesting spectral characteristics. Would energy transfer to chl $a$ /PSII happen in this mutant?

There are quite a few questions that need to be answered about the PBS. First, what is the function of the $\alpha$ AP-B subunit? It doesn't seem to have a major role within the PBS. Bryant believes that it acts as the terminal emitter to PSI (Bryant, personal communication). I have already done some preliminary work with epr to investigate that possibility using the apcD mutant. If one blocks the electron transfer pathway using DCMU, one can quantify the amount of PSI excited by measuring the ferredoxin signal in the epr spectrum. I excited whole cells at $77 \mathrm{~K}$ with $580 \mathrm{~nm}$ light to directly excite PC of the PBS without exciting much chl $a$. I monitored the ferredoxin signal and illuminated the sample a second time with blue light to excite all of the chl $a$. I could quantify the changes in the ferredoxin signal to see if different amounts of excitation energy reach the PSI reaction center in the wild-type cells versus the apcD cells. I still need to optimize the 
illumination times, but the preliminary work looked promising with the apcD mutant showing impaired energy transfer to PSI.

I had a lot of problems in quantifying the amounts of phycobiliprotein present in whole cells. A technique for the specific bleaching of phycobiliproteins in vivo has been developed for Synechococcus 6301 (Zhao and Brand, 1989). The method uses heating at $60{ }^{\circ} \mathrm{C}$ to bleach the phycobiliproteins without altering the chlorophyll or carotenoid absorption. One should be able to calculate fluorescence quantum yields for the whole cells more precisely using this method.

Another direction that could be explored is the preparation of PSII-PBS complexes for all of the mutants. The spectral data should be easier to interpret than for whole cells. Also, one could monitor the amount of oxygen evolved by the preparations as a gauge of the efficiency of the antenna. Preparations have already been worked out for Synechococcus elongatus (Kashino, Enami, et al., 1992), thermophilic Synechococcus sp (Kura-Hotta, Satoh, et al., 1986), Porphyridium cruentum (Clement-Metral and Gantt, 1983;

Chereskin and Gantt, 1985; Clement-Metral, Gantt, et al., 1985; Chereskin and Gantt, 1986), and Anacystis nidulans (Pakrasi and Sherman,1984). The preparations appear to be difficult to work with, but the results could be very interesting since one could measure of the efficiency energy transfer from the PBS to the reaction centers.

Also, I think we need to address the question of how the PBS complex binds to the membrane. One approach to solving this problem would be to use crosslinkers to bind adjacent proteins in a 
PBS-PSII preparation, isolate the crosslinked proteins using a denaturing gel, cleave the crosslinker using light, and identify the pieces. The composition of the PBS is relatively well identified, so it would be only a matter of identifying the proteins from the membrane. 
References for Chapter 6

Chereskin, B. M., J. D. Clement-Metral, and E. Gantt (1985). Plant Physiol. 77: 626-629.

Chereskin, B. M. and E. Gantt (1986). Plant Cell Physiol. 27: 751-754.

Clement-Metral, J. D. and E. Gantt (1983). FEBS Lett. 156: 185-188.

Clement-Metral, J. D., E. Gantt, and T. Redlinger (1985). Arch. Biochem. Biophys. 238: 10-17.

Kashino, Y., I. Enami, S. Igarashi, and S. Katoh (1992). Plant Cell Physiol. 33: 259-266.

Kura-Hotta, M., K. Satoh, and S. Katoh (1986). Arch. Biochem. Biophys. 249: 1-7.

Maxson, P., Sauer, K., J. Zhou, D. A. Bryant, and A. N. Glazer (1989). Biochim. Biophys. Acta 977: 40-51.

Pakrasi, H. B. and L. A. Sherman (1984). Plant Physiol. 74: 742-745.

Rhie, G. and S. I. Beale (1992). J. Biol. Chem. 267: 16088-16093.

Zhao, J. and J. J. Brand (1989). Arch. Microbiol. 152: 447-452. 

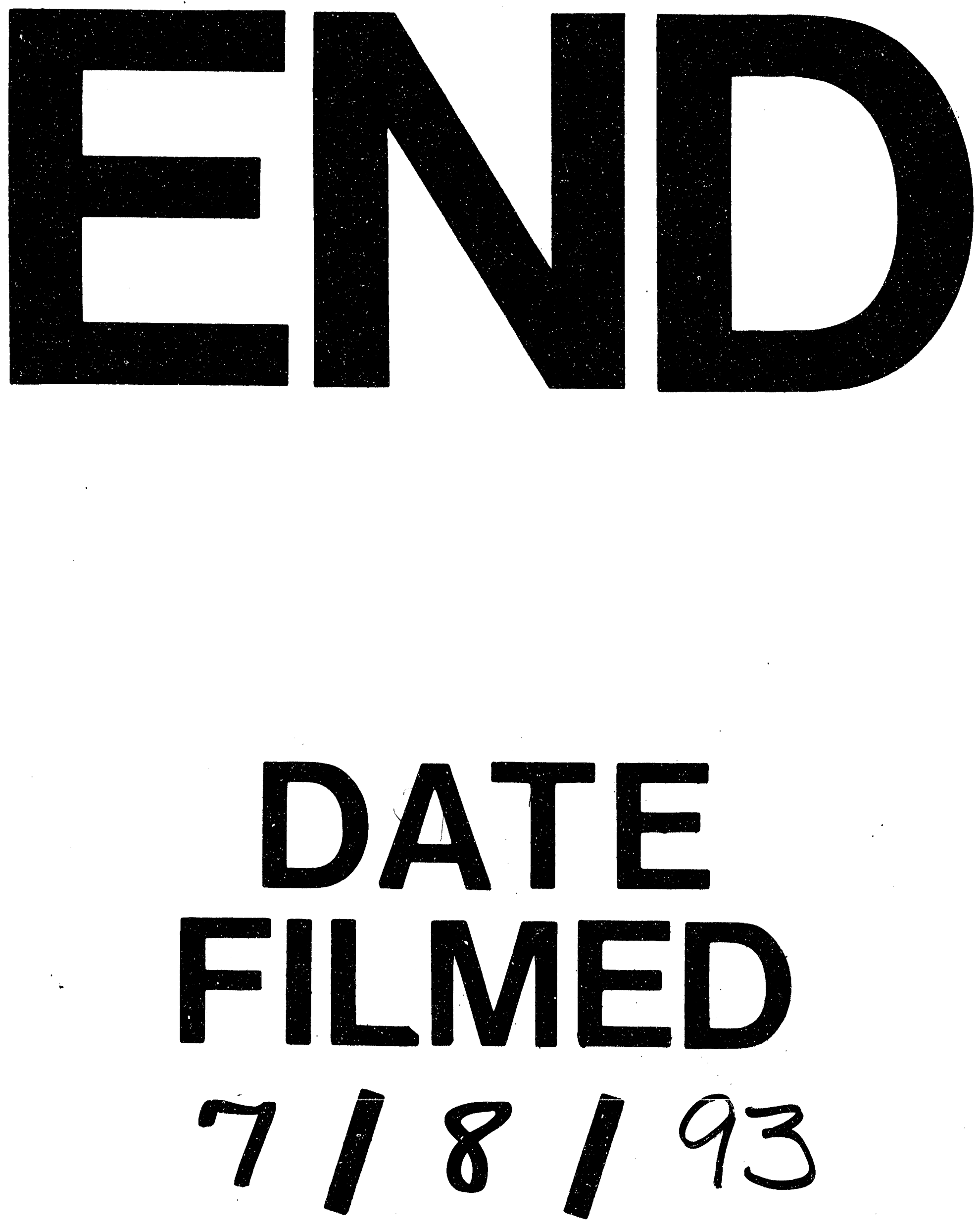
